Assurances et gestion des risques

Insurance and Risk Management

ASSURANCES

\title{
Développement d'un modèle de notation de crédit économétrique général pour des financements de projet
}

\section{Simon Rhainds}

Volume 85, numéro 3-4, décembre 2018

URI : https://id.erudit.org/iderudit/1056947ar

DOI : https://doi.org/10.7202/1056947ar

Aller au sommaire du numéro

\section{Éditeur(s)}

Faculté des sciences de l'administration, Université Laval

\section{ISSN}

1705-7299 (imprimé)

2371-4913 (numérique)

Découvrir la revue

\section{Citer cet article}

Rhainds, S. (2018). Développement d’un modèle de notation de crédit économétrique général pour des financements de projet. Assurances et gestion des risques / Insurance and Risk Management, 85(3-4), 225-302. https://doi.org/10.7202/1056947ar

\section{Résumé de l'article}

Dans cet article, nous décrivons le processus de développement d'un modèle de notation de crédit de financement de projet. Tout d'abord, nous présentons chacune des étapes du développement du modèle menant à la sélection des facteurs quantitatifs et macroéconomiques pertinents dans l'établissement des cotes de crédit des financements de projet. Ensuite, des facteurs qualitatifs, dont les poids sont déterminés par jugement d'expert, sont ajoutés au modèle quantitatif afin d'obtenir le modèle final. Plusieurs tests de performance sont effectués sur ce modèle afin de valider le choix des facteurs et le poids de chacun d'eux dans le modèle. L'un de ces tests consiste à comparer la performance du modèle final à celle de plusieurs modèles alternatifs dont les poids des facteurs qualitatifs sont déterminés aléatoirement. Nous constatons que le modèle final surpasse la majorité des modèles alternatifs et surpasse grandement le modèle contenant seulement les facteurs quantitatifs et macroéconomiques.
Tous droits réservés $\odot$ Faculté des sciences de l'administration, Université Laval, 2019
Ce document est protégé par la loi sur le droit d'auteur. L'utilisation des services d'Érudit (y compris la reproduction) est assujettie à sa politique d'utilisation que vous pouvez consulter en ligne.

https://apropos.erudit.org/fr/usagers/politique-dutilisation/ 


\section{DÉVELOPPEMENT D'UN MODÈLE DE NOTATION DE CRÉDIT ÉCONOMÉTRIQUE GÉNÉRAL POUR DES FINANCEMENTS DE PROJET}

Simon RHAINDS

\section{RÉSUMÉ}

Dans cet article, nous décrivons le processus de développement d'un modèle de notation de crédit de financement de projet. Tout d'abord, nous présentons chacune des étapes du développement du modèle menant à la sélection des facteurs quantitatifs et macroéconomiques pertinents dans l'établissement des cotes de crédit des financements de projet. Ensuite, des facteurs qualitatifs, dont les poids sont déterminés par jugement d'expert, sont ajoutés au modèle quantitatif afin d'obtenir le modèle final. Plusieurs tests de performance sont effectués sur ce modèle afin de valider le choix des facteurs et le poids de chacun d'eux dans le modèle. L'un de ces tests consiste à comparer la performance du modèle final à celle de plusieurs modèles alternatifs dont les poids des facteurs qualitatifs sont déterminés aléatoirement. Nous constatons que le modèle final surpasse la majorité des modèles alternatifs et surpasse grandement le modèle contenant seulement les facteurs quantitatifs et macroéconomiques. 


\section{INTRODUCTION}

La cote de crédit d'une contrepartie est un intrant important lorsqu'il s'agit de gestion de risque d'investissement, plus précisément de gestion de risque de crédit. Cette cote donne notamment de l'information sur la probabilité de défaut et sur la perte en cas de défaut de ladite contrepartie. Ces deux éléments sont essentiels pour bien évaluer le risque de crédit d'un portefeuille. Il existe deux types de cote de crédit: les cotes de crédit externes et les cotes de crédit internes. Les cotes de crédit externes sont publiques et données par des agences de notation indépendantes comme Standard \& Poor's (S\&P), Moody's, DBRS et Fitch. À l'inverse, les cotes de crédit internes sont calculées par la compagnie et utilisées seulement par celle-ci.

Dans la majorité des cas, les cotes de crédit externes sont seulement disponibles pour les contreparties importantes (Hull, 2010). Il est donc important pour une institution financière d'avoir un moyen d'évaluer correctement le risque de ses contreparties non cotées. De plus, le Banking Committee on Banking Supervision de 2006 suggère aux institutions financières et aux banques d'utiliser des modèles de notation de crédit internes.

L'un des types d'investissement pour lesquels les cotes de crédit externes sont très rarement disponibles sont les financements de projet. Lorsque le portefeuille d'une institution financière comprend des financements de projet non notés, il est important de développer un modèle de notation de crédit pour ce type d'investissement. Ce dernier est un modèle économétrique qui réplique à plus ou moins deux crans les cotes de risque des agences de notation externes. Afin de pouvoir développer un tel modèle, on doit répondre à la question suivante :

Quels sont les facteurs quantitatifs, macroéconomiques et qualitatifs pertinents dans un modèle de notation fantôme de financement de projet? Quels sont les poids de ces facteurs dans le modèle?

Afin de répondre à cette question, il est important de bien comprendre ce qu'est le financement de projet. C'est pour cela qu'une brève introduction à ce type d'investissement est faite dans la section 3. Mais avant tout, une revue de littérature sur les modèles de notation de crédit est présentée dans la section 2. Par la suite, les différentes approches économétriques de développement de modèles de notation qui existent ainsi que les exigences d'un modèle de notation sont présentées dans la section 4. Ensuite, les informations nécessaires au développement du modèle en fonction de l'approche choisie en 4 et 
le traitement des données sont présentées à la section 5. Dans la section suivante (section 6), les différentes étapes du développement du modèle, ainsi que les résultats intermédiaires seront présentés. Dans la section 7 , les résultats finaux sont présentés. Finalement la conclusion sera présentée à la section 8 . La bibliographie est dans la section 9. Une annexe détaillant certaines étapes de ce travail est disponible sur demande.

\section{ReVue de LitTÉrature ET BONNES PRATIQUES}

Les cotes de crédit ont pour but de mesurer la solvabilité des entreprises. Elles représentent l'opinion d'une agence de notation qui évalue la force fondamentale de crédit d'un émetteur et sa capacité à satisfaire pleinement et ponctuellement ses obligations de dette (Gonzales et al. (2004), Berblinger (1996) et Wappenschmidt (2009)). Selon Krahnen (2001), c'est une pratique courante pour les banques d'approuver des prêts et du crédit à l'aide de modèles internes de type "notation de crédit fantôme». Ceci découle de l'accord de Bâle II (2004) qui dit que, pour le risque de crédit, les banques peuvent employer différents mécanismes d'évaluation:

- La méthode dite "standard" consiste à utiliser des systèmes de notation fournis par des organismes externes (agences de notation).

- Les méthodes plus sophistiquées (méthodes IRB) avec la méthode dite IRB-fondation et celle dite IRB-avancée impliquent des méthodologies internes et propres à l'établissement financier d'évaluation de cotes ou de notes, afin de peser le risque relatif du crédit.

Les cotes de crédit sont principalement influencées par trois catégories de déterminants. Dans la première catégorie, on retrouve les ratios financiers et les informations financières. Selon Ederington (1985), ces facteurs approximent les facteurs spécifiques de l'entreprise comme le levier, la liquidité et la taille de celle-ci. La deuxième catégorie comprend les mécanismes de gouvernance de la firme, qui selon Bhojraj \& Sengupta (2003), inclue des facteurs comme la structure de propriété et l'indépendance du conseil d'administration. La dernière catégorie de déterminants de la cote de crédit est constituée des facteurs macroéconomiques. Ces derniers peuvent être, par exemple, selon Amato \& Furfine (2004), la croissance de PIB. D'autres facteurs comme l'inflation, les taux d'intérêt, etc. peuvent également être considérés dans cette catégorie. En pratique, il y a plusieurs méthodes statistiques 
utilisées afin de tester si les facteurs compris dans les catégories ci-haut ont réellement un pouvoir explicatif sur la qualité de crédit. Matthies (2013) relate que les ratios financiers sont essentiels pour déterminer la qualité de crédit d'une entreprise. Les deux autres catégories peuvent être vues comme de l'information complémentaire à ces derniers.

Dans Matthies (2013), il est dit que les méthodes statistiques d'estimation de la qualité de crédit peuvent être séparées en deux catégories, les méthodes ordonnées et les méthodes non-ordonnées. La première catégorie comprend la méthode des moindres carrés ordinaires (MCO) et les modèles probit ordonnés. Cette catégorie de modèle repose sur l'hypothèse principale que les cotes de crédit sont classées. La deuxième catégorie, quant à elle, comprend des méthodes comme les modèle probit non-ordonnés et les analyses discriminantes linéaires. L'hypothèse que les cotes ne sont pas classées est faite dans ces modèles. Dans une étude empirique, Ederington (1985) trouve que les modèles probit ordonnés surpassent les modèles de type MCO.

\section{DESCRIPTION DES FINANCEMENTS DE PROJET}

Cette section est une introduction au concept de financement de projet. Il commence avec un bref historique expliquant l'origine des financements de projet. Par la suite, une présentation de la structure et des différents intervenants d'un financement de projet sera faite.

\subsection{Historique}

Les méthodes de financement de projets sont utilisées depuis très longtemps. Par exemple, en 1299, lorsque la Couronne anglaise a voulu financer l'exploration et le développement de la mine d'argent Devon, elle a fait appel à la banque d'affaires de florentine, Frescodalbi, qu'elle remboursait directement avec la production de la mine. Par la suite, dans les années 1600, le financement de projet fut utilisé pour financer les expéditions commerciales maritimes. En effet, au retour d'une expédition, les marchandises et les navires étaient vendus et le profit séparé entre les investisseurs. Ce bref historique des financements de projet est tiré de Kensinger (1993). Les techniques de financement ont beaucoup évolué depuis ce temps, mais les fondements restent les mêmes. De nos jours, les financements de projet sont utilisés pour financer la construction de centrales électriques, de routes à péage, d'hôpitaux, 
etc. Les principales catégories de financement de projet utilisées par S\&P, qui sont aussi les catégories utilisées dans l'industrie en général, sont les suivantes:

- Projets d'énergie

- Centrale hydroélectrique

- Centrale solaire

- Centrale éolienne

- Projets d'infrastructure

- Route à péage

- Pont

- Produits chimiques et gaz naturel liquéfié

- Pétrochimie

- Partenariat public-privé/Initiative de financement privé (PPP/PFI)

- Établissement scolaire

- Bâtiment public

- Ressources Naturelles

- Pétrole et gaz

- Exploitation minière

- Générique

Afin d'être cohérent avec l'industrie, c'est également la classification qui est utilisée pour notre modèle de notation interne.

\subsection{Définition et structure}

Afin de pouvoir développer un modèle de notation pour une industrie, il est important de bien comprendre cette dernière. Comme il est difficile de donner une définition précise de ce qu'est un financement de projet, une définition générale, tirée de Gardner (2012), est plutôt présentée:

the raising of finance on a Limited Recourse basis, for the purposes of developing a large capital-intensive infrastructure project, where the borrower is a special purpose vehicle and repayment of the financing by the borrower will be dependent on the internally generated cashflows of the project. 
Un autre aspect important du financement de projet est la structure. Le schéma 1 donne une bonne vue d'ensemble de cette dernière et des différentes parties qui interviennent dans le financement de projet. Ce schéma est tiré de Caranci (2011).

\section{FIGURE 1 Schéma de la structure simplifiée d'un financement} de projet typique
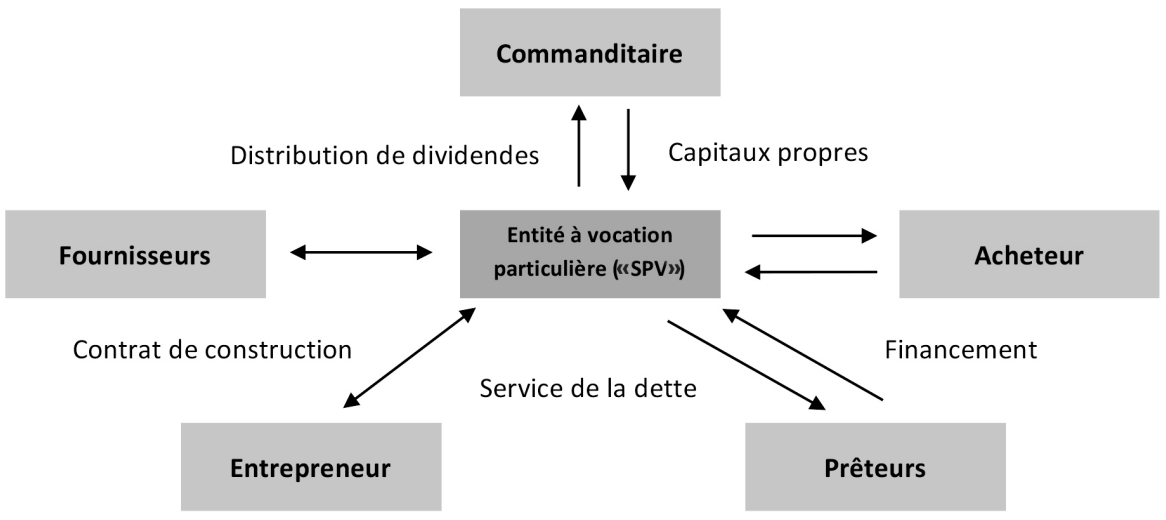

On trouve ici-bas les définitions des différents intervenants d'un financement de projet présents dans la figure 1 .

Commanditaire: Les sponsors d'un projet sont normalement propriétaires du projet et ont une part des actions plus ou moins grande. Dans la majorité des cas, le sponsor est une seule compagnie (gouvernement). Par contre, dans certains cas, plusieurs compagnies peuvent se rassembler afin de financer un même projet. Comme la dette d'un financement de projet est dans la majorité des cas sans recours, le sponsor ne met pas directement à risque les avoirs de sa compagnie mère, donc le sponsor est rémunéré par la distribution de dividendes. La distribution des dividendes est parfois régie par un contrat stipulant qu'un certain niveau de service de la dette doit être respecté.

Entité à vocation particulière: Dans la majorité des financements de projet, une entité ad hoc (SPV) est créée par le ou les sponsors dans le seul but de réaliser le projet. La SPV n'a qu'une seule source de revenu, soit les revenus du projet.

Entrepreneur: L'entrepreneur est celui qui est responsable de la réalisation du projet. Il est souvent choisi en fonction du niveau de difficulté de la construction en question. Tout dépendant de la 
complexité de la construction, l'entrepreneur principal peut faire appel à des sous-contractants ou tout assumer lui-même. Dans certains cas l'entrepreneur principal possédera des actions dans la SPV.

Opérateur: L’opérateur du projet est responsable de la phase d'opération. Plus précisément, il s'assure du bon fonctionnement du projet à la suite de la construction. Il est également responsable de l'entretien des actifs du projet. L'opérateur, tout comme l'entrepreneur, est choisi en fonction du niveau de complexité que représente l'opération du projet. Également, tout comme l'entrepreneur, il n'est pas impossible qu'il possède des actions dans la SPV.

Fournisseurs: Les fournisseurs sont ceux qui fournissent les intrants importants au fonctionnement du projet. Pour donner un exemple concret: le fournisseur d'une centrale électrique au charbon est la compagnie qui fournirait le charbon.

Acheteur: L'acheteur est le parti qui achète la production du projet. Dans certains cas, une entente d'achat régissant les quantités et les prix que l'acheteur s'engage à acheter dans le futur peut avoir été signée. Dans d'autre cas, le projet peut être exposé totalement aux risques de marché et à la compétition.

Prêteurs: Les prêteurs sont ceux qui possèdent la dette du projet sous toutes ses formes. Ils sont ceux qui sont exposés au risque de défaut du projet. En 2013, il y avait, selon Gevero (2014), 7596 projets non cotés pour 462 projets cotés. On comprend donc que la majorité de la dette émise par les SPV des financements de projet est de la dette privée. Dans certains cas, un garant ou un assureur garantit le paiement total ou partiel au détenteur de dette.

\section{DÉVELOPPEMENT DE MODÈLE DE NOTATION}

Les différentes approches économétriques de développement de modèle de notations de crédit ainsi que les exigences minimales que doit respecter le modèle développé sont présentées dans cette section. Tout d'abord, les bonnes pratiques en développement de modèle de notation interne sont exposées. Ensuite, les deux approches économétriques de développement de modèle de notation les plus souvent utilisées seront présentées. Il s'agit de l'approche Bon-Mauvais, en anglais Good-Bad (GB) et de l'approche de Notation de crédit fantôme, soit Shadow Rating (SR) en anglais. 


\subsection{Bonnes pratiques en développement de modèle de notation de crédit}

Un modèle de notation de crédit développé à l'interne, même si seulement utilisé à l'interne doit respecter certaines exigences minimales dictées par Bâle II (2004). Un résumé des exigences les plus importantes est fait dans Zaalberg (2013). Voici le résumé:

- Un modèle de notation doit être fondé sur l'expérience historique et des preuves empiriques. Les données historiques doivent être représentatives du portefeuille à noter.

- Il devrait intégrer à la fois des données historiques et le jugement d'experts.

- Les données historiques sur lesquelles le modèle est basé doivent avoir un historique d'au moins un cycle économique, soit environ cinq ans.

- Les cotes de crédit du modèle doivent être en ligne avec les cotes de crédit externes des agences de notation.

- Le modèle doit être robuste, c'est-à-dire qu'il doit performer dans l'échantillon de développement et hors de l'échantillon.

- Le modèle doit être intuitif et cohérent avec le jugement expert.

\subsection{Bon-Mauvais}

L'approche Bon-Mauvais est basée sur la régression logistique (Varekamp, 2014) dont la théorie est présentée dans la première partie de cette sous-section. Par la suite, l'approche de développement de modèle de notation Bon-Mauvais est introduite.

\subsubsection{Régression logistique}

Comme la variable dépendante dans ce type de régression est binaire, les méthodes conventionnelles de régression par moindre carrés ordinaires ne peuvent pas être utilisées. La théorie des modèles linéaires généralisés est plutôt utilisée. Un modèle linéaire généralisé consiste en trois éléments:

1. Une distribution de probabilité de la famille exponentielle.

2. Un prédicteur linéaire $\eta=X \beta$, où $\eta$ est le prédicteur linéaire, $X$ sont les variables indépendantes et $\beta$ est le vecteur des paramètres. 
3. Une fonction de liaison $g$ telle que $\mathrm{E}(\mathrm{Y})=\mu=g^{-1}(\eta)$, où Y est la variable dépendante, $\mathrm{E}(\mathrm{Y})$ est l'espérance de $\mathrm{Y}, \mu$ est la moyenne de la distribution de probabilité exponentielle et $g$ est la fonction de liaison.

Dans le cas de la régression logistique, les trois éléments sont les suivants :

1. Une distribution de Bernoulli.

2. $\eta$ est le logarithme de la probabilité que la variable Y de la distribution de Bernoulli soit égale à 1 .

3. La fonction de liaison $g(\mu)$ est

$$
g(\mu)=\ln \left(\frac{\mu}{1-\mu}\right)
$$

De cela, il est possible de déduire que la fonction d moyenne $g^{-1}$ est

$$
g^{-1}(\mathrm{X})=\mu=\frac{e^{\mathrm{X} \beta}}{1-e^{\mathrm{X} \beta}} .
$$

Cette fonction $g^{-1}(\mathrm{X})$ donne la probabilité que la variable aléatoire $\mathrm{Y}$ soit égale à 0 . Donc, $1-g^{-1}(\mathrm{X})$ est la probabilité que la variable $\mathrm{Y}=1$. Ce qui donne

$$
\mathrm{P}(\mathrm{Y}=1)=1-g^{-1}(\mathrm{X})=\frac{1}{1+e^{\mathrm{X \beta}}} .
$$

De l'équation (3), il est possible d'estimer le vecteur $\beta$ avec la méthode du maximum de vraisemblance. En d'autres mots, les coefficients $\beta$ sont optimisés afin de maximiser la probabilité de la variable dépendante observée.

\subsubsection{Approche «Bon-mauvais»}

Comme il a été mentionné plus haut, l'approche Bon-Mauvais repose sur la régression logistique. Plus précisément, l'approche Good-Bad est faite en régressant des facteurs $\mathrm{X}$ contenant de l'information sur la solvabilité des contreparties sur une variable dépendante $\mathrm{Y}$ prenant la valeur "Bon" ou 0 si la contrepartie ne fait pas défaut dans l'année suivant la date d'observation des facteurs et "Mauvais" ou 1 si la contrepartie fait défaut dans l'année suivant la date d'observation des facteurs. En utilisant le maximum de vraisemblance, il est donc possible 
de trouver les facteurs $\mathrm{X}$ qui contiennent le plus d'information possible sur la solvabilité d'une contrepartie. Par la suite, on peut estimer la probabilité de défaut d'une contrepartie avec les $\hat{\beta}$ estimés avec l'équation

$$
\hat{\mathrm{P}}(\mathrm{Y}=1)=\frac{1}{1+e^{\mathrm{x} \hat{\beta}}} .
$$

Cette probabilité de défaut peut ensuite être reliée à une cote de crédit interne.

\subsection{Notation de crédit fantôme}

L'approche Notation de crédit fantôme, quant à elle, est plutôt basée sur la théorie de la régression linéaire. Les deux parties de cette sous-section ont la même structure que celle de la sous-section 4.2.

\subsubsection{Régression linéaire}

Dans la théorie sur la régression linéaire, on fait l'hypothèse que la variable dépendante présente une relation linéaire avec les variables explicatrices. L'équation d'une régression linéaire prend la forme suivante:

$$
y=\alpha+\beta_{1} x_{1}+\beta_{2} x_{2}+\beta_{3} x_{3}+\beta_{4} x_{4}+\ldots+\beta_{n} x_{n}+\varepsilon
$$

où,

$\mathrm{y}=$ le vecteur des $\mathrm{j}$ observations de variables dépendantes,

$\alpha=$ une constante,

$\mathrm{x}_{\mathrm{i}}=$ le vecteur des $\mathbf{j}$ observations de variables indépendantes $\mathrm{i}$,

$\beta_{\mathrm{i}}=$ le coefficient de la variable indépendante $i$,

$\varepsilon=$ un vecteur des erreurs bruit blanc d'estimation des $j$ observation des variables dépendantes y.

La même équation peut être réécrite en notation matricielle comme suit:

$$
\mathrm{Y}=\alpha+\mathrm{X} \beta+\varepsilon
$$

où

$\mathrm{Y}=\left[\begin{array}{l}y_{1} \\ \ldots \\ y_{j}\end{array}\right], \alpha=\left[\begin{array}{c}\alpha \\ \ldots \\ \alpha\end{array}\right], \mathrm{X}=\left[\begin{array}{ccc}x_{1,1} & \ldots & x_{1, n} \\ \ldots & \ldots & \ldots \\ x_{j, 1} & \ldots & x_{j, n}\end{array}\right], \beta=\left[\begin{array}{c}\beta_{1} \\ \ldots \\ \beta_{n}\end{array}\right]$ et $\varepsilon=\left[\begin{array}{c}e_{1} \\ \ldots \\ e_{j}\end{array}\right]$. 
L'estimation des coefficients $\beta$ est faite en minimisant la somme du carré des erreurs du vecteur $\varepsilon$. C'est la méthode des moindres carrés ordinaires. Avec cette méthode les coefficients $\beta$ et la constante $\alpha$ sont estimés suivant:

$$
\begin{gathered}
\hat{\beta}=\left(X^{\mathrm{T}} \mathrm{X}\right)^{-1} \mathrm{X}^{\mathrm{T}} \mathrm{Y} . \\
\hat{\alpha}=\overline{\mathrm{Y}}=\overline{\mathrm{X}} \hat{\beta} .
\end{gathered}
$$

\subsubsection{Approche «Notation de crédit fantôme»}

L'approche Notation de crédit fantôme repose sur la théorie de la régression linéaire. Cette approche est utilisée lorsque le nombre de défauts est limité et lorsque les cotes de crédit des principales agences de notation sont disponibles pour une part importante et représentative du portefeuille. L'objectif de cette approche est de choisir les facteurs de risque et les poids de ces facteurs de façon à imiter le mieux possible les cotes de crédit externes quand il n'y a pas suffisamment de données pour construire un modèle de prédiction de défaut explicite (Erlenmaier, 2011). Les cotes externes utilisées peuvent venir de plusieurs agences, mais il est important d'avoir une méthode permettant de toutes les ramener sur une échelle commune.

On remarque dans le graphique de la figure 2 que la relation entre les probabilités de défaut et les cotes de crédit semblent exponentielle. $\mathrm{Si}$ on regarde plutôt le graphique à la figure 2 , qui montre les logarithmes naturels des probabilités de défaut en fonction des cotes de crédit, on remarque une relation plus linéaire. C'est pour cette raison qu'au lieu de régresser les probabilités de défaut des observations directement sur les variables explicatives et une constante, les logarithmes naturels de ces probabilités sont régressés sur les variables explicatives et une constante. De plus, en appliquant cette transformation, l'effet des probabilités de défaut élevées est atténué. La formule de régression est donc la suivante:

$$
\operatorname{Ln}(\mathrm{DP})=\alpha+\mathrm{X} \beta+\varepsilon
$$

où la matrice $\mathrm{X}$ est la matrice des facteurs de risques observés. De l'équation 9, il est donc possible d'obtenir

$$
\mathrm{DP}=e^{\alpha+\mathrm{x} \beta+\varepsilon} .
$$

Les coefficients $\beta$ et la constante $\alpha$ seront estimés avec les estimateurs des moindres carrés ordinaires (OLS) présentés plus tôt dans le texte. 
FIGURE 2 Graphique des probabilités de défaut annuelles moyennes des grandes sociétés (PD) en fonction des cotes de risque de Moody's

La période d'échantillonnage va de 1999 à 2014. Les données sont tirées de la base de données de défaut et de recouvrement de Moody's.

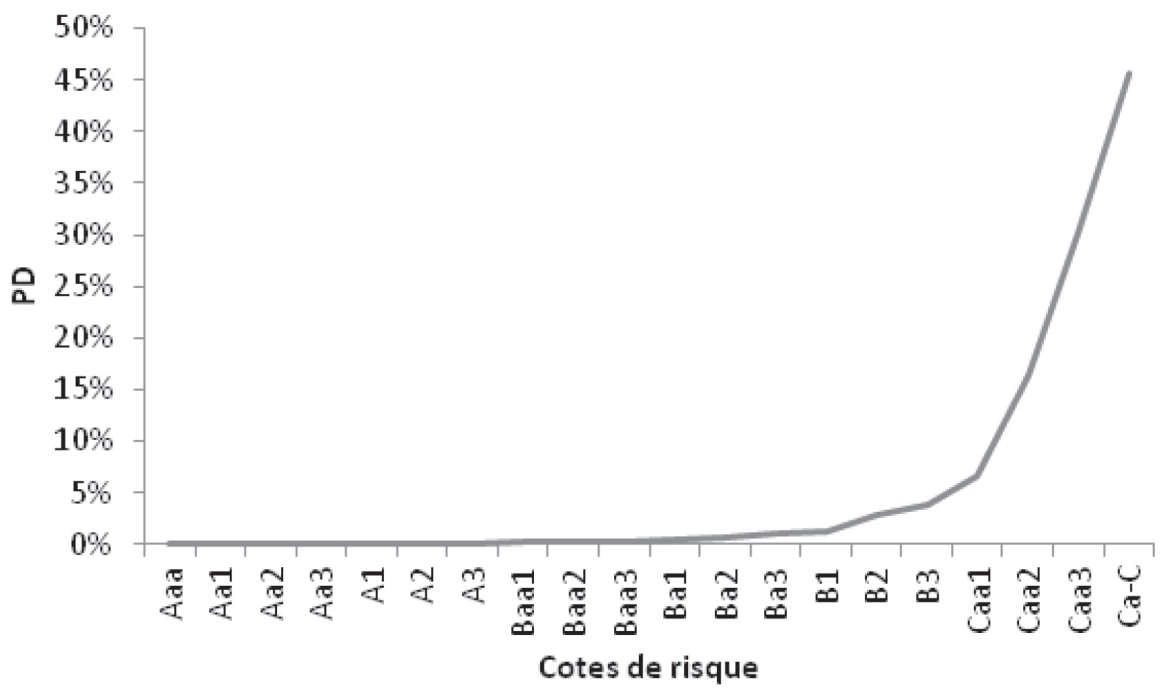

FIGURE 3 Graphique des logarithmes naturel des probabilités de défaut annuelles moyennes des grandes sociétés (PD) en fonction des cotes de risque de Moody's

La période d'échantillonnage va de 1999 à 2014. Les données sont tirées de la base de données de défaut et de recouvrement de Moody's.

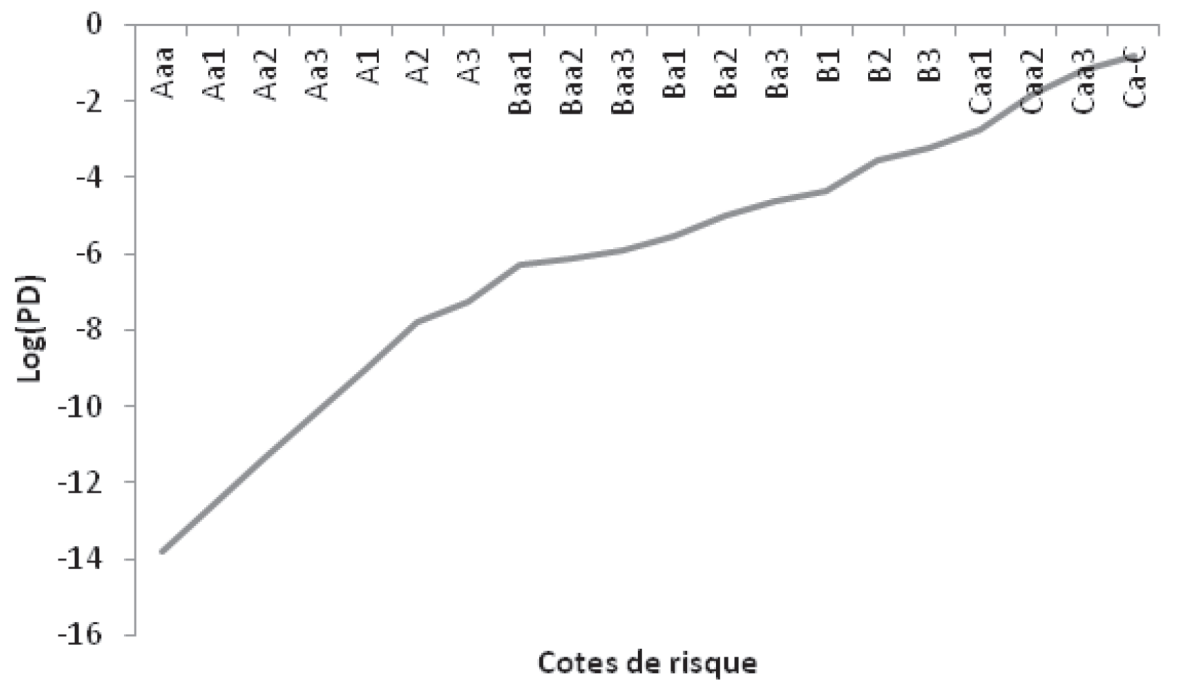




\subsection{Approche choisie}

Après une analyse de l'échantillon des financements de projet, il est choisi d'utiliser l'approche Notation de crédit fantôme. Les arguments en faveur de cette approche sont exposés et expliqués dans cette section. Les points faibles de l'approche sont ensuite présentés.

\subsubsection{Points forts}

Selon Izzi (2012), afin de pouvoir développer un modèle Good-Bad, on doit avoir un échantillon de développement contenant au moins 30 défauts par variable explicative et un échantillon de validation représentant au moins $10 \%$ de l'échantillon de développement. La première piste de solution envisagée pour développer un modèle BonMauvais est de développer ce dernier avec les projets présents dans le portefeuille de l'institution financière. Les données financières ainsi que les défauts des projets sont facilement accessibles. Sinon un échantillon externe représentatif du portefeuille peut être utilisé.

Le nombre de financements de projet et de défauts dans un portefeuille sont donc deux aspects importants si on veut développer un modèle Bon-Mauvais. Un portefeuille, ou un échantillon, ne contenant pas assez de projets pour pouvoir créer un échantillon de développement, représentant au maximum $90 \%$ de tous les projets, suffisamment important pour obtenir des résultats économétriques crédibles ne peut donc pas être utilisé pour développer un modèle Bon-Mauvais. L'approche Bon-Mauvais doit également être écartée dans le cas où le portefeuille, ou l'échantillon, ne contient pas au moins 30 défauts. Dans des cas semblables, l'approche Notation de crédit fantôme est plutôt envisagée.

Toujours selon Izzi (2012), afin de développer un modèle Notation de crédit fantôme, il faut avoir un portefeuille, ou un échantillon, constitué d'au moins 150 contreparties notées par des agences de notation externes afin de pouvoir créer un échantillon de développement d'au moins 100 contreparties notées et un échantillon de validation d'au moins 50 contreparties notées. L'échantillon utilisé dans cet essai contient plus de 150 contreparties notées, mais seulement un défaut. C'est donc pour cette raison que l'approche Notation de crédit fantôme est choisie. 


\subsubsection{Points faibles}

Toute approche économétrique a ses forces et ses faiblesses. Dans le cas de la Notation de crédit fantôme, la principale force est qu'il est possible de développer un modèle de notation à partir d'un échantillon ne contenant pas beaucoup de défauts. Par contre, ce modèle a également ses faiblesses et il est important de les reconnaitre afin de pouvoir adéquatement interpréter les résultats. Premièrement, il faut savoir que le pouvoir discriminant de cette méthode est étroitement lié à la qualité des cotes de crédit externe de l'échantillon. Par le fait même, le pouvoir discriminant de l'approche est plus faible que celui de l'approche BonMauvais, qui lui repose directement sur les défauts des contreparties. Le deuxième point faible de la méthode Notation de crédit fantôme est que les probabilités de défaut en découlant sont, en moyenne, trop optimistes. Ceci est dû à l'inégalité de Jensen qui dit que si $\mathrm{X}$ est une variable aléatoire et $\varphi$ une fonction convexe, alors

$$
\varphi(\mathrm{E}[\mathrm{X}]) \geq \mathrm{E}[\varphi(\mathrm{X})] .
$$

En se référant à l'équation 9, on remarque que c'est le $\ln (\mathrm{PD})$ qui est estimé dans le modèle. Comme le logarithme naturel est une fonction convexe, il est possible de conclure que

$$
\ln (\mathrm{E}[\mathrm{PD}]) \geq \mathrm{E}[\ln (\mathrm{PD})]
$$

et en utilisant l'équation 10 on obtient l'inégalité suivante

$$
\mathrm{E}[\mathrm{PD}] \geq e^{\mathrm{E}[\ln (\mathrm{PD})]} \text {. }
$$

De l'inégalité donnée par l'équation (13) on conclut que les probabilités de défauts estimées par un modèle $S R$ sont, en moyenne, trop petites. Cet effet est par contre atténué lorsque les probabilités de défaut estimées sont par la suite ramenées sur une échelle alphanumérique.

Avant d'aller plus loin, il faut introduire le concept du Shadow Accuracy ratio (SAR), communément appelé en français le ratio de précision de la méthode Notation de crédit fantôme. Ce ratio est tiré d'Izzi (2012).

\subsubsection{Ratio de précision de la méthode «Notation de crédit fantôme»}

Le SAR est fortement inspiré du coefficient de Gini (Gini, 1912). L'intuition derrière le SAR est de comparer le pouvoir explicatif du facteur à un facteur avec un pouvoir explicatif parfait. Plus le pouvoir explicatif du facteur est proche de celui avec un pouvoir parfait, meilleur est le facteur. 
La première étape du calcul du SAR consiste à classer en ordre croissant les facteurs transformés. Il est important de se rappeler que les facteurs ont été transformés de façon à ce qu'un facteur avec une valeur près de 0 soit associé à une grande $\mathrm{PD}$ et vice versa. Ensuite, pour chaque facteur ordonné, la somme des PD des observations avec des valeurs de facteurs plus petites ou égales est calculée. Ces sommes cumulatives sont par la suite divisées par la somme totale des PD. On peut par la suite calculer pour chaque point, le pourcentage des observations qui ont une valeur du facteur inférieure ou égale au point en question. De cette façon, on obtient une série de paires de points avec laquelle on peut tracer une courbe. La deuxième étape consiste à ordonner indépendamment en ordre croissant les valeurs des facteurs et les PD. De cette façon, on obtient les observations que l'on aurait observées si le facteur avait eu un pouvoir discriminant parfait. Le même processus que précédemment est alors répété, mais avec ce nouvel échantillon. Une troisième courbe est alors ajoutée, cette courbe est une ligne droite du point $(0,0)$ au point $(1,1)$. Elle représente la courbe d'un facteur qui n'a aucun pouvoir explicatif.

Dans la partie suivante du texte, un exemple numérique simple est présenté afin d'illustrer le concept du SAR. Dans l'exemple suivant, le SAR d'un facteur quelconque est calculé.

\section{TABLEAU 1 Échantillon fictif de cinq observations utilisées pour illustrer le calcul du SAR}

Pour chaque observation on trouve l'ID, le score du facteur, la probabilité de défaut (PD) et la probabilité de défaut avec pouvoir de discrimination parfait. Les observations sont classées en ordre croissant de score. Les probabilités de défaut avec pouvoir de discrimination parfait sont les PD classées en ordre croissant.

\begin{tabular}{c|c|c|c}
\hline ID & SCORE DU FACTEUR & \multicolumn{2}{|c|}{ PD } \\
\hline 1 & 2 & 0,20 & ${ }^{*} D_{\text {Pouvoir de discrimination parait }}$ \\
\hline 2 & 3 & 0,05 & 0,20 \\
\hline 3 & 4 & 0,20 & 0,20 \\
\hline 4 & 7 & 0,05 & 0,10 \\
\hline 5 & 8 & 0,10 & 0,05 \\
\hline
\end{tabular}

On observe que les PD de la dernière colonne du tableau ci-haut sont en ordre décroissant et que les scores du facteur en ordre croissant. Cela serait la réalité si le facteur testé ici avait un pouvoir discriminant parfait. L'étape suivante consiste à calibrer les trois courbes, soit la courbe du SAR, la courbe avec aucun pouvoir explicatif et la courbe avec pouvoir discriminant parfait. Pour ce faire, pour chaque 
observation, la proportion des observations avec un score plus petit ou égal au score de l'observation est représentée sur l'axe des X et la proportion des défauts résultant est tracée sur l'axe des Y. Les points suivants en résultent:

\section{TABLEAU 2 Détermination des points $(X, Y)$ de l'échantillon fictif $d u$ Tableau 1 pour un ensemble de scores équidistant sur l'intervalle [1,10]}

Les observations $S A R$ sont les paires d'observations réelles. Les paires d'observations sans pouvoir sont construites avec l'équation $X=Y$. Les paires d'observations avec pouvoir parfait sont construites en ordonnant les $X$ et les $Y$ en ordre croissant et en agencent les $X$ et les $Y$ ayant la même position. Tous les scores de 1 à 10 sont conservés.

\begin{tabular}{|c|c|c|c|c|c|c|}
\hline SCORE & $\mathrm{X}_{\mathrm{SAR}}$ & $\mathrm{Y}_{\mathrm{SAR}}$ & $\mathrm{X}_{\text {Sans pouvoir }}$ & $Y_{\text {Sans pouvoir }}$ & $X_{\text {Pouvoir parfait }}$ & $Y_{\text {Pouvoir parfait }}$ \\
\hline 1 & 0,00 & 0,00 & 0,00 & 0,00 & 0,00 & 0,00 \\
\hline 2 & 0,20 & 0,33 & 0,20 & 0,20 & 0,20 & 0,33 \\
\hline 3 & 0,40 & 0,42 & 0,40 & 0,40 & 0,40 & 0,67 \\
\hline 4 & 0,60 & 0,75 & 0,60 & 0,60 & 0,60 & 0,83 \\
\hline 5 & 0,60 & 0,75 & 0,60 & 0,60 & 0,60 & 0,83 \\
\hline 6 & 0,60 & 0,75 & 0,60 & 0,60 & 0,60 & 0,83 \\
\hline 7 & 0,80 & 0,83 & 0,80 & 0,80 & 0,80 & 0,92 \\
\hline 8 & 1,00 & 1,00 & 1,00 & 1,00 & 1,00 & 1,00 \\
\hline 9 & 1,00 & 1,00 & 1,00 & 1,00 & 1,00 & 1,00 \\
\hline 10 & 1,00 & 1,00 & 1,00 & 1,00 & 1,00 & 1,00 \\
\hline
\end{tabular}

À partir de ces résultats on peut obtenir les points reliés à notre échantillon en ne gardant que les paires $(\mathrm{X}, \mathrm{Y})$ uniques.

\section{TABLEAU 3 Ensemble des $(X, Y)$ de l'échantillon fictif du tableau 1 en ne gardant que les paires uniques}

Les observations SAR sont les paires d'observations réelles. Les paires d'observations sans pouvoir sont construites avec l'équation $X=Y$. Les paires d'observations avec pouvoir parfait sont construites en ordonnant les $X$ et les $Y$ en ordre croissant et en agençant les $X$ et les $Y$ ayant la même position. Seulement les paires $(X, Y)$ avec des scores présents dans le tableau 1 sont conservées.

\begin{tabular}{|c|c|c|c|c|c|}
\hline $\mathrm{X}_{\mathrm{SAR}}$ & $\mathrm{Y}_{\mathrm{SAR}}$ & $X_{\text {Sans pouvoir }}$ & $Y_{\text {Sans pouvoir }}$ & $X_{\text {Pouvoir parfait }}$ & $Y_{\text {Pouvoir parfait }}$ \\
\hline 0,00 & 0,00 & 0,00 & 0,00 & 0,00 & 0,00 \\
\hline 0,20 & 0,33 & 0,20 & 0,20 & 0,20 & 0,33 \\
\hline 0,40 & 0,42 & 0,40 & 0,40 & 0,40 & 0,67 \\
\hline 0,60 & 0,75 & 0,60 & 0,60 & 0,60 & 0,83 \\
\hline 0,80 & 0,83 & 0,80 & 0,80 & 0,80 & 0,92 \\
\hline 1,00 & 1,00 & 1,00 & 1,00 & 1,00 & 1,00 \\
\hline
\end{tabular}


Le graphique du SAR peut maintenant être tracé.

\section{FIGURE 4 La courbe réelle du SAR, la courbe sans pouvoir explicatif} et la courbe avec pouvoir parfait de l'échantillon fictif du tableau 1

La courbe verte représente les paires $(X, Y)$ des observations avec un pouvoir explicatif parfait. La courbe bleue représente les paires $(X, Y)$ des observations réelles. La courbe rouge représente les paires $(X, Y)$ des observations sans pouvoir discriminant.

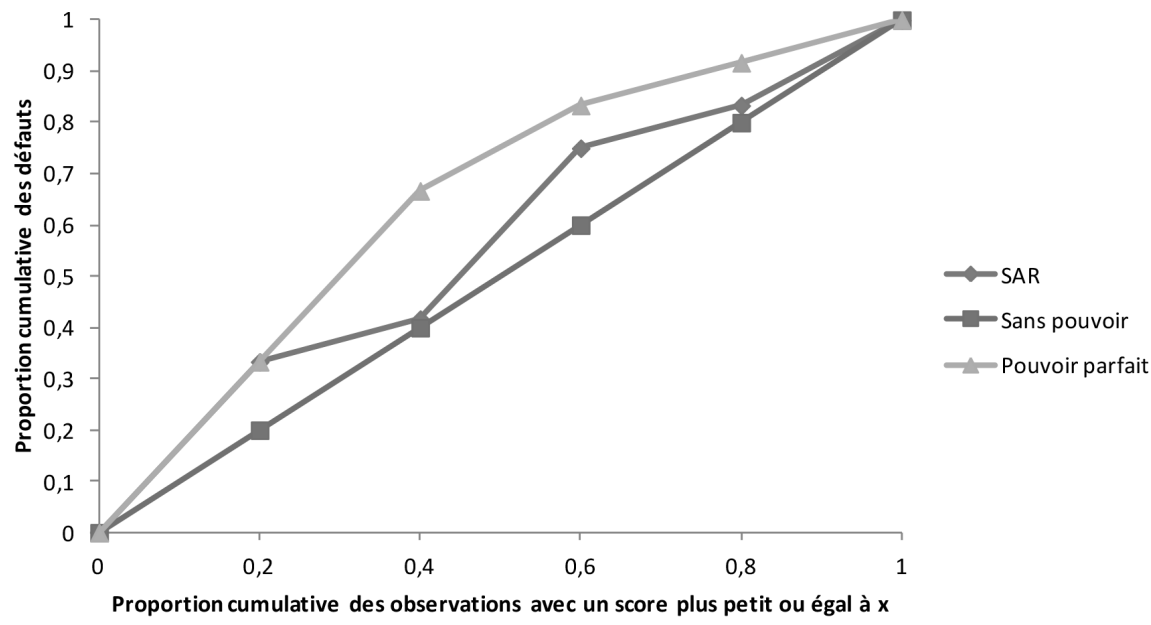

Il est maintenant possible de calculer le ratio de l'aire entre la courbe "SAR» et "Sans pouvoir» sur l'aire entre la courbe «Pouvoir parfait» et "Sans pouvoir». Dans le cas présent, les aires sont respectivement 0,067 et 0,150 , ce qui donne un SAR de 0,444. Le SAR d'un facteur possédant un facteur de discrimination parfait serait de 1. À l'inverse, un facteur avec un pouvoir de discrimination nul aurait un SAR de 0.

Afin d'interpréter le SAR, un parallèle avec une autre mesure doit être fait. Il s'agit de l'aire sous la courbe de caractéristique du récepteur opérant (ASCCRO) qui est en fait une simple transformation du SAR. Pour passer de l'ASCCRO au SAR, il faut appliquer la transformation suivante:

$$
\mathrm{SAR}=\frac{\text { ASCCRO }_{\text {SAR }}-0,5}{\text { ASCCRO }_{\text {Pouvoir parfait }}-0,5}
$$

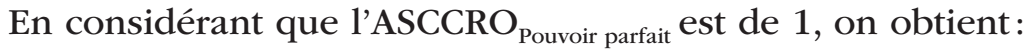

$$
\mathrm{SAR}=\frac{\text { ASCCRO }_{\mathrm{SAR}}-0,5}{0,5}
$$


Finalement, en simplifiant l'équation ci-dessus, on obtient:

$$
\mathrm{SAR}=2 * \mathrm{ASCCRO}_{\mathrm{SAR}}-1
$$

La règle d'interprétation de l'ASCCRO est tirée du système traditionnel de pointage académique:

- 0,90-1,00 = Excellent (A)

- $0,80-0,90=$ Bien $(B)$

- $0,70-0,80$ = Passable $(\mathrm{C})$

- $0,60-0,70=$ Faible $(\mathrm{D})$

- $0,50-0,60$ = Échec $(F)$

Si l'on transforme cette règle d'interprétation pour le SAR, on obtient:

- $0,80-1,00=$ Excellent (A)

- $0,60-0,80=$ Bien $(B)$

- $0,40-0,60=$ Passable $(\mathrm{C})$

- 0,20-0,40 = Faible (D)

- $0,00-0,20$ = Échec $(F)$

On peut donc interpréter un SAR de 0,444 comme ayant une note de $\mathrm{C}$ et donc comme étant passable.

\section{PréSentation des ÉtAPeS de dÉVELOPPEMENT DU MODĖLE}

Selon Erlenmaier (2011), les étapes principales du développement d'un modèle économétrique de type notation de crédit fantôme sont les suivantes:

1. Calibrage des notations externes

2. Construction de l'échantillon de données externes

3. Analyse univariée des facteurs

4. Analyse multivariée des facteurs

5. Analyse d'impact

6. Documentation 
Afin d'être transparent sur la méthode de développement du modèle, une étape intermédiaire, nommée "Traitement des données", est ajoutée entre les étapes 2 et 3. De plus, la portion "Analyse d'impact» n'est pas traitée dans cet essai car le but de ce dernier se limite à déterminer les facteurs et leur poids. La structure des étapes modifiée est comme suit:

1. Calibration des notations externes

2. Construction de l'échantillon de données externes

3. Traitement des données

4. Analyse univariée des facteurs

5. Analyse multivariée des facteurs

6. Documentation

Dans cette section, chacune des étapes est expliquée.

\subsection{Calibration des notations externes}

Comme un modèle de type notation de crédit fantôme est basé sur les cotes de crédit externes, il est important d'inclure le plus de cotes externes à l'échantillon. Pour ce faire, il faut trouver une méthode pour relier entre elles les cotes de crédit des différentes agences de notation.

Comme les probabilités de défaut varient en fonction de la cote de crédit et en fonction de la séniorité de la dette, il est souhaitable de comparer les cotes des différents émetteurs à travers une seule catégorie de la dette afin de faire abstraction de l'effet des différentes séniorités. Il est donc primordial d'avoir une méthode permettant de comparer les cotes de crédit de différentes séniorités. Il est également nécessaire de pouvoir transformer les cotes de crédit en probabilité de défaut.

\subsection{Construction de l'échantillon de données externes}

Une fois les méthodologies de calibration de cotes de crédit externe trouvées, il faut maintenant construire un échantillon. Toujours selon Erleinmaier (2011), les quatre questions qu'il faut se poser lors de la construction des échantillons sont:

1. Quels types d'échantillon sont nécessaires?

2. Comment peut-on construire ces échantillons? 
3. Est-ce que des informations ont plus de poids de d'autre?

4. Quelle est la structure de corrélation de l'échantillon?

\subsection{Traitement des données}

Les données recueillies à l'étape précédente sont certainement hétérogènes et ont besoin d'être nettoyées et travaillées afin de les rendre plus uniformes. À cette étape les données aberrantes sont éliminées. Des transformations, tel que des changements de domaine, pourraient être appliquées aux données à cette étape afin de minimiser l'effet des données extrêmes et de rendre comparables entre eux les coefficients de régression.

\subsection{Analyse univariée des facteurs}

À cette étape le pouvoir explicatif des facteurs sur les PD est testé individuellement. Le SAR et la significativité du $\beta$ de la régression $\log (\mathrm{PD})=\alpha+\beta \mathrm{X}+\epsilon$ sont les deux critères de sélection à cette étape. Le SAR est un critère couramment utilisé dans l'évaluation de modèle de notation de crédit et c'est pour cette raison qu'il est inclu dans nos critères de sélection. La significativité du $\beta$, tant qu'à elle, donne une bonne idée de la signification statistique du facteur. Suite à cette étape, une portion des facteurs sera éliminée.

\subsection{Analyse multivariée des facteurs}

À cette étape, le pouvoir explicatif combiné des différents facteurs retenus à l'analyse univariée est testé. Il est donc possible de prendre en considération l'interaction qu'il pourrait y avoir entre les différents facteurs. Les combinaisons de facteurs avec les pouvoirs explicatifs combinés les plus forts sont retenus.

\subsection{Documentation}

Pour des raisons de conformité et de transparence, les étapes et méthodes utilisées pour le développement du nouveau modèle doivent être documentées. Cet essai fera guise de documentation. 


\section{6. ÉTAPES DE DÉVELOPPEMENTS DU MODÈLE}

Dans cette section, les étapes du développement du modèle sont présentées. Les étapes sont présentées dans l'ordre suivant: la transformation des cotes de crédit en probabilité de défaut; la construction de l'échantillon externe ainsi que l'analyse de ce dernier; les transformations appliquées aux données brutes de l'échantillon; les étapes et les résultats de l'analyse univariée et de l'analyse multivariée; la transformation finale des probabilités de défaut extraite des modèles en cote de crédit; puis, la sélection du modèle final.

Comme les données sur les facteurs qualitatifs sont seulement disponibles pour quelques financements de projet, le nombre de données pour lequel nous avons de l'information qualitative est insuffisant pour développer un modèle économétrique significatif. Afin d'éliminer le biais potentiel résultant d'un échantillon trop petit, l'analyse des données quantitatives et l'analyse des données qualitatives sont faites séparément.

\subsection{Transformations des notations externes}

Afin de ramener les cotes de crédit des différentes agences de crédit sur une échelle numérique commune, la table 4 est utilisée. Cette dernière est développée à partir du tableau de correspondance des cotes de crédit de la banque TD ${ }^{1}$.

TABLEAU 4 Table de conversion des cotes de crédit des grandes agences de notation DBRS, Moody's et S\&P développée à partir du tableau de correspondance des cotes de crédit de la banque TD

\begin{tabular}{c|c|c|c}
\hline ÉCHELLE NUMÉRIQUE & \multicolumn{2}{|c|}{ MOODY'S } & DBPS \\
\hline 1 & Aaa & AAA & AAA \\
\hline 2 & Aa1 & AA+ & AAH \\
\hline 3 & Aa2 & AA & AA \\
\hline 4 & Aa3 & AA & AAL \\
\hline 5 & A1 & A & AH \\
\hline 6 & A2 & A & A \\
\hline 7 & A3 & A & AL \\
\hline
\end{tabular}




\begin{tabular}{|c|c|c|c|}
\hline ÉCHELLE NUMÉRIQUE & MOODY'S & S\&P & DBRS \\
\hline 8 & Baa1 & $\mathrm{BBB}+$ & BBBH \\
\hline 9 & Baa2 & BBB & BBB \\
\hline 10 & Baa3 & BBB- & BBBL \\
\hline 11 & Ba1 & $\mathrm{BB}+$ & $\mathrm{BBH}$ \\
\hline 12 & $\mathrm{Ba} 2$ & BB & BB \\
\hline 13 & Ва3 & BB- & BBL \\
\hline 14 & B1 & $\mathrm{B}+$ & $\mathrm{BH}$ \\
\hline 15 & B2 & B & B \\
\hline 16 & B3 & B- & $\mathrm{BL}$ \\
\hline 17 & Caa1 & $\mathrm{CCC}+$ & $\mathrm{CCCH}$ \\
\hline 18 & Caa2 & CCC & $\mathrm{CCC}$ \\
\hline 19 & Caa3 & $\mathrm{CCC}-$ & $\mathrm{CCCL}$ \\
\hline 20 & $\mathrm{Ca}$ & CC & CC \\
\hline 21 & C & C & C \\
\hline
\end{tabular}

Afin de ramener les cotes de crédit de différentes séniorités à une même séniorité (Senior Unsecured) l'algorithme de Moody's "Moody's Senior Ratings Algorithm \& Estimated Senior Ratings" présenté dans Gupta (2009) est utilisé. Les motivations derrière l'utilisation de cet algorithme sont les suivantes:

1) L'échelle numérique utilisée plus haut est sur 21 cotes, tout comme celle de Moody's.

2) Moody's est l'une des agences de notation les plus importante.

3) Les probabilités de défaut utilisées sont calculées à partir de la base de données de défaut et de recouvrement de Moody's.

4) Seulement l'algorithme de Moody's était disponible.

L'algorithme consiste à un nombre de crans qu'il faut appliquer à une cote non senior non garantie afin d'obtenir la cote senior non garantie (cote de l'émetteur). 
TABLEAU 5 Algorithme Moody's tiré de Gupta (2009) pour obtenir la cote senior non garantie à partir de la cote d'une obligation avec une autre séniorité

\begin{tabular}{|c|c|c|c|c|c|c|c|}
\hline $\begin{array}{c}\text { COTE DE } \\
\text { L'OBLIGATION }\end{array}$ & $\begin{array}{l}\text { SENIOR } \\
\text { GARANTIE }\end{array}$ & $\begin{array}{l}\text { COTE DE } \\
\text { LA FAMILLE } \\
\text { DE SOCIÉTEÉS }\end{array}$ & $\begin{array}{l}\text { SENIOR NON } \\
\text { GARANTIE / } \\
\text { COTE DE } \\
\text { L'ÉMETTEUR }\end{array}$ & $\begin{array}{l}\text { SENIOR } \\
\text { SUBOR- } \\
\text { DONNÉE }\end{array}$ & $\begin{array}{l}\text { JUNIOR } \\
\text { SUBOR- } \\
\text { DONNÉE }\end{array}$ & $\begin{array}{l}\text { SUBOR- } \\
\text { DONNÉE }\end{array}$ & $\begin{array}{l}\text { EMPRUNT } \\
\text { BANCAIRE } \\
\text { SYNDIQUÉ }\end{array}$ \\
\hline Aaa & -1 & 0 & 0 & 0 & 0 & 0 & -2 \\
\hline Aa1 & -1 & 0 & 0 & +1 & +1 & +1 & -2 \\
\hline Aa2 & -1 & 0 & 0 & +1 & +1 & +1 & -2 \\
\hline Aa3 & -1 & 0 & 0 & +1 & +1 & +1 & -2 \\
\hline $\mathrm{A} 1$ & -1 & 0 & 0 & +1 & +1 & +1 & -2 \\
\hline A2 & -1 & 0 & 0 & +1 & +1 & +1 & -2 \\
\hline A3 & -1 & 0 & 0 & +1 & +1 & +1 & -2 \\
\hline Baa1 & -1 & 0 & 0 & +1 & +1 & +1 & -2 \\
\hline Baa2 & -1 & 0 & 0 & +1 & +1 & +1 & -2 \\
\hline Baa3 & -1 & 0 & 0 & +1 & +1 & +1 & -2 \\
\hline Ba1 & -1 & -1 & 0 & +1 & +1 & +1 & -2 \\
\hline $\mathrm{Ba} 2$ & -2 & -1 & 0 & +1 & +1 & +1 & -2 \\
\hline Ba3 & -2 & -1 & 0 & +1 & +1 & +1 & -2 \\
\hline B1 & -2 & -1 & 0 & +1 & +1 & +1 & -2 \\
\hline B2 & -2 & -1 & 0 & +2 & +2 & +2 & -2 \\
\hline B3 & -2 & -1 & 0 & +2 & +2 & +2 & -2 \\
\hline Caa1 & -2 & -1 & 0 & +2 & +2 & +2 & -2 \\
\hline Caa2 & -2 & -1 & 0 & +2 & +2 & +2 & -2 \\
\hline Caa3 & -2 & -1 & 0 & +2 & +2 & +2 & -2 \\
\hline $\mathrm{Ca}$ & -1 & -1 & 0 & +2 & +2 & +2 & -1 \\
\hline C & 0 & 0 & 0 & +2 & +2 & +2 & 0 \\
\hline
\end{tabular}

*Algorithme tiré de Moody's Senior Ratings Algorithm \& Estimated Senior Ratings

Pour ce qui est de transformer les cotes de crédit en probabilité de défaut, la matrice de transition annuelle des grandes sociétés est utilisée. Cette matrice est créée à partir de la base de données de défaut et de recouvrement de Moody's sur un historique allant de 1999 à 2014. Les probabilités de défaut par cote de risque tirées de la table de transition sont présentées dans le tableau 6. Les motivations derrière l'utilisation de cette dernière se trouvent dans une annexe disponible sur demande. 
TABLEAU 6 Table de conversion des cotes de crédit Moody's en probabilité de défaut (PD) fait à partir de la base de données de défauts et de recouvrement de Moody's sur un historique allant de 1999 à 2014 et utilisant les données des grandes entreprises présentes dans la base de données

\begin{tabular}{l|c} 
Aaa & $0,00010 \%$ \\
\hline Aa1 & $0,00033 \%$ \\
\hline Aa2 & $0,00110 \%$ \\
\hline Aa3 & $0,00367 \%$ \\
\hline A1 & $0,01220 \%$ \\
\hline A2 & $0,04056 \%$ \\
\hline A3 & $0,07012 \%$ \\
\hline Baa1 & $0,18919 \%$ \\
\hline Baa2 & $0,21341 \%$ \\
\hline Baa3 & $0,27343 \%$ \\
\hline Ba1 & $0,40182 \%$ \\
\hline Ba2 & $0,68586 \%$ \\
\hline Ba3 & $0,98132 \%$ \\
\hline B1 & $1,29893 \%$ \\
\hline B2 & $2,83666 \%$ \\
\hline B3 & $3,92112 \%$ \\
\hline Caa1 & $6,53316 \%$ \\
\hline Caa2 & $16,34589 \%$ \\
\hline Caa3 & $30,13595 \%$ \\
\hline Ca-C & $45,57029 \%$ \\
\hline & \\
\hline
\end{tabular}

\subsection{Construction de l'échantillon de données externes}

\subsubsection{Données quantitatives}

Maintenant que les transformations des notations externes sont traitées, on peut s'attarder à la construction de l'échantillon de données externes. Avant de pouvoir construire la base de données des informations financières, il faut choisir quels ratios financiers sont testés dans 
la construction du modèle. Le tableau 7 contient les ratios financiers utilisés par les agences de notations pour noter des financements de projet ainsi que le niveau d'accessibilité et la source de chacun. Tous les ratios présents dans le tableau se retrouvent également dans Matthies (2013). Le niveau d'accessibilité est déterminé de la façon suivante:

- 1 = Le ratio est calculé directement avec les états financiers et les informations sont toujours claires et disponibles.

- 2 = Le calcul du ratio nécessite des calculs supplémentaires et les informations nécessaires au calcul de ce dernier ne sont pas toujours claires et disponibles.

- 3 = Le calcul du ratio nécessite des projections financières

\section{TABLEAU 7 Table des ratios financiers utilisés par les agences de notations pour noter des financements de projet avec leur niveau d'accessibilité et la source leur correspondant}

Le niveau d'accessibilité de 1 est attribué quand le ratio est calculé directement avec les états financiers et que les informations sont toujours claires et disponibles. Le niveau d'accessibilité de 2 est attribué quand le calcul du ratio nécessite des calculs supplémentaires et les informations nécessaires au calcul de ce dernier ne sont pas toujours claires et disponibles dans l'état financier. Le niveau d'accessibilité de 3 est attribué quand le calcul du ratio nécessite des projections financières.

\begin{tabular}{|c|c|c|}
\hline NOM DU RATIO & $\begin{array}{c}\text { NIVEAU } \\
\text { D'ACCESSIBILITÉ* }\end{array}$ & SOURCES \\
\hline $\begin{array}{l}\text { Ratio de couverture du service de la dette } \\
\text { (DSCR) }\end{array}$ & 1 & $\begin{array}{l}\text { Caranci (2011), Deluz (2010), Lendberg (2014) } \\
\text { et Selting (2014) }\end{array}$ \\
\hline $\begin{array}{l}\text { Ratio de marge brute d'autofinancement } \\
\text { sur dette (FFO/Debt) }\end{array}$ & 2 & Deluz (2010) \\
\hline Ratio des flux de trésorerie sur la dette & 1 & Caranci (2011) \\
\hline $\begin{array}{l}\text { Ratio des flux de trésorerie moins les } \\
\text { dépenses en immobilisations sur la dette }\end{array}$ & 2 & Caranci (2011) \\
\hline Ratio de l'EBITDA sur charges d'intérêts & 1 & Caranci (2011) et Fic (2014) \\
\hline $\begin{array}{l}\text { Coefficient de couverture sur la durée } \\
\text { du prêt (LLCR) }\end{array}$ & 3 & $\begin{array}{l}\text { Delfour (2009), Caranci (2011) } \\
\text { et Selting (2014) }\end{array}$ \\
\hline $\begin{array}{l}\text { Coefficient de couverture sur la durée } \\
\text { du projet (PLCR) }\end{array}$ & 3 & $\begin{array}{l}\text { Delfour (2009), Lendberg (2014) } \\
\text { et Selting (2014) }\end{array}$ \\
\hline $\begin{array}{l}\text { Coefficient de couverture sur la durée } \\
\text { de la concession (CLCR) }\end{array}$ & 3 & Deluz (2010) \\
\hline $\begin{array}{l}\text { Ratio de la dette par rapport aux } \\
\text { capitaux propre }\end{array}$ & 1 & Caranci (2011), Deluz (2010) et Delfour (2009) \\
\hline Ratio de la dette par rapport à l'EBITDA & 1 & Fic (2014) \\
\hline
\end{tabular}


Comme il est pratiquement impossible d'obtenir les projections financières de toutes les contreparties de l'échantillon, les ratios avec un niveau d'accessibilité de 3 ont été éliminés. Les ratios avec un niveau d'accessibilité de 2 ont également été éliminés car l'information nécessaire à leur calcul n'est pas disponible dans tous les états financiers. La liste résultant des ratios à tester est présentée dans le tableau 8. On retrouve dans ce tableau le ratio de couverture du service de la dette (DSCR), le ratio des flux de trésorerie sur la dette, le ratio de la dette par rapport aux bénéfices avant intérêts, impôts et amortissement, le ratio de la dette par rapport aux capitaux propre et le ratio des bénéfices avant intérêts, impôts et amortissement sur charges d'intérêts. À partir de maintenant, nous allons référer aux bénéfices avant intérêts, impôts et amortissement et aux bénéfices avant intérêts, impôts comme étant l'EBITDA et l'EBIT.

\section{TABLEAU 8 Ratios financiers résultant de l'élimination des ratios avec des niveaux d'accessibilité égaux à 2 et 3 du Tableau 7 , de leur définition et du signe attendu de leur relation avec les probabilités de défaut}

Un signe attendu négatif suppose que la relation qui existe entre le ratio et la probabilité de défaut est négative. Un signe attendu positif suppose le contraire.

\begin{tabular}{l|l|c}
\multicolumn{2}{c}{ RATIO } & \multicolumn{2}{c}{ DÉFINITION } \\
$\begin{array}{l}\text { Ratio de couverture du service de la dette } \\
\text { (DSCR) }\end{array}$ & $\begin{array}{l}\text { EBITDA / (Charges d'intérêts + Paiements } \\
\text { de capital) }\end{array}$ & \\
\hline Ratio des flux de trésorerie sur la dette & $\begin{array}{l}\text { Flux monétaires des opérations / Dette } \\
\text { portant intérêt }\end{array}$ & - \\
\hline Ratio de la dette par rapport à l'EBITDA & Dette portant intérêt / EBITDA & - \\
\hline $\begin{array}{l}\text { Ratio de la dette par rapport aux capitaux } \\
\text { propre }\end{array}$ & Dette portant intérêt / Capitalisation totale & + \\
\hline Ratio de l'EBITDA sur charges d'intérêts & EBITDA / Charges d'intérêts & - \\
\hline
\end{tabular}

D'autres ratios utilisés par l'agence de notation DBRS tirés de Holman (2012) sont également inclus dans la liste des ratios à tester. Ces derniers sont présentés dans le tableau 9. 
TABLEAU 9 Table des ratios financiers ratios utilisés par DBRS tirés de Holman (2012), de leur définition et du signe attendu de leur relation avec les probabilités de défaut

Un signe attendu négatif suppose que la relation qui existe entre le ratio et la probabilité de défaut est négative. Un signe attendu positif suppose le contraire.

\begin{tabular}{|c|c|c|}
\hline RATIO & DÉFINITION & SIGNE ATTENDU \\
\hline Ratio de couverture des intérêts & EBIT / Charges d'intérêts & - \\
\hline Rendement de l'actif & Résultat net / Total des Actifs & - \\
\hline Marge EBIT & EBIT / Revenus Totaux & - \\
\hline Marge EBITDA & EBITDA / Revenus Totaux & - \\
\hline Ratio du fonds de roulement & Actifs courants / Passifs courants & - \\
\hline Ratio de capacité de gain de base & EBIT / Actifs & - \\
\hline Ratio de capacité de gain de base & EBITDA / Actifs & - \\
\hline
\end{tabular}

Dans les tableaux ci-haut on retrouve également le signe attendu de la relation entre la PD et le ratio. Par exemple, pour le DSCR, on s'attend à ce qu'une grande valeur de DSCR corresponde à une faible PD. La relation inverse est attendue pour un ratio comme le ratio de la dette par rapport à l'EBITDA.

À l'aide de ce tableau, un gabarit d'éléments d'un état financier nécessaires au calcul des ratios a été créé. Dans le cas où un projet est en construction, les projections financières sont utilisées afin de remplir le gabarit. Un exemple de ce gabarit rempli est présenté au tableau 10.

TABLEAU 10 Exemple abrégé d'une ligne du gabarit créé avec les composantes des ratios financiers sélectionnés présenté dans les tableaux 8 et 9

\begin{tabular}{|c|c|c|c|c|c|c|c|c|c|c|c|}
\hline NOM & DEVISE & ANNEE_EF & & TOTAL_REV & RESUL- & T_NET & CHARGES_INT & EBITDA & EBIT & CFO & \\
\hline$A B C D$ & CAD & 2014 & $\ldots$ & 1000 & 10 & 0 & 200 & 500 & 400 & 600 & $\ldots$ \\
\hline \multicolumn{2}{|c|}{ CURR_LT_DT } & \multicolumn{2}{|c|}{ DETTE_INT } & \multicolumn{2}{|c|}{ ACTIFS_COURANTS } & PAS: & FS_COURANTS & \multicolumn{2}{|l|}{$\mathrm{CP}$} & \multicolumn{2}{|c|}{ ACTIFS } \\
\hline & 200 & \multicolumn{2}{|c|}{10000} & \multicolumn{2}{|l|}{200} & & 300 & \multicolumn{2}{|c|}{20000} & \multicolumn{2}{|c|}{30000} \\
\hline
\end{tabular}


L'étape suivante consiste à remplir le gabarit avec des données financières des financements de projet cotés afin de pouvoir créer l'échantillon de développement et de validation. Par contre, comme les financements de projet sont souvent des entités privées, leurs données financières sont difficilement accessibles. De plus, une grande quantité de ces derniers n'ont pas de cote de crédit externe. Il faut donc trouver des projets qui sont à la fois cotés et dont les états financiers sont disponibles. Ces deux conditions que doivent respecter les projets de l'échantillon font en sorte que l'univers des financements de projet pouvant être utilisé dans le développement du modèle est grandement réduit. Voici donc les étapes de construction de la base de données financières de financements de projet.

1) Création d'une liste de projets qui ont une cote de crédit externe.

2) Création de l'historique des cotes de crédit externes disponibles.

3) Création d'une liste de projets avec des informations financières (états financiers) disponibles.

4) Création de l'historique des informations financières disponibles.

5) Création de la liste finale des année/projet pour lesquels la cote de crédit et les informations financières sont disponibles.

6) Remplir le gabarit présenté précédemment.

Voici donc les statistiques de l'échantillon créé:

TABLEAU 11 Tableau du nombre de contreparties et du nombre de contrepartie-années présentes dans l'échantillon

Une contrepartie-année correspond à une année d'état financier pour un financement de projet. Par exemple, l'état financier 2015 du projet X correspond à une contrepartie-année et l'état financier 2014 du même projet $\mathrm{X}$ correspond à une autre contrepartie-année.

NOMBRE DE CONTREPARTIES

119
NOMBRE DE CONTREPARTIE-ANNÉE

599

Erlenmaier (2011) souligne que les échantillons de développement et de validation doivent être représentatifs du portefeuille à coter. La création de l'échantillon de développement et de l'échantillon de validation à partir de l'échantillon coté est faite de façon à ce que la répartition géographique et la répartition par secteur soient semblables à celles de l'échantillon interne à coter. 
La figure ci-dessous présente les statistiques de l'échantillon interne non coté, de l'échantillon coté de développement et de l'échantillon coté de validation. On remarque que l'échantillon interne non-coté a une très forte concentration au Canada et aux États-Unis. Comme les deux autres échantillons n'ont pas une assez grande concentration dans ces deux pays, l'hypothèse que l'économie de l'Angleterre se rapproche assez de celle du Canada et des États-Unis est faite. Cette hypothèse repose sur le fait que les trois pays font partie des 15 pays les plus développés dans le monde (Jahan, 2015). On remarque également que les trois échantillons contiennent des projets australiens.

On note également que la majorité des projets dans les trois échantillons sont des projets d'énergie. Ensuite, les PPP/PFI et les projets d'infrastructure occupent les deuxième et troisième places dans les trois échantillons. Il y a donc trois échantillons comparables.

\section{FIGURE 5 Comparaison de statistiques descriptives de l'échantillon interne non coté, de l'échantillon coté de développement et de l'échantillon coté de validation}

Pour chaque sous échantillon, la répartition par pays et par secteur est illustrée. Les trois sous échantillons ont des distributions par pays et par secteur semblable. Cette figure est construite à partir de l'échantillon de développement du modèle et de l'échantillon de projets sans cote de crédit pour lesquels le modèle doit calculer une cote de crédit.

\begin{tabular}{|c|c|c|}
\hline \multicolumn{2}{|l|}{ Externe Coté Validation } & Externe Coté Développement \\
\hline PAYS & $\%$ & PAYS \\
\hline Canada/États-Unis/Royaume-Uni & $64 \%$ & Canada/États-Unis/Royaume-Uni \\
\hline Australie & $12 \%$ & Australie \\
\hline Mexique & $4 \%$ & Mexique \\
\hline Autre & $20 \%$ & Autre \\
\hline SECTEUR & $\%$ & SECTEUR \\
\hline Projets d'énergie & $32 \%$ & Projets d'énergie \\
\hline $\mathrm{PPP} / \mathrm{PFI}$ & $28 \%$ & $\mathrm{PPP} / \mathrm{PFI}$ \\
\hline Projets d'infrastructure & $12 \%$ & Projets d'infrastructure \\
\hline Ressources Naturelles & $12 \%$ & Ressources Naturelles \\
\hline Produits chimiques et gaz naturel liquéfié & $8 \%$ & Produits chimiques et gaz naturel liquéfié \\
\hline Générique & $8 \%$ & Générique \\
\hline
\end{tabular}

$\begin{array}{ccc} & \text { Interne Non-Coté } & \\ \% & \text { PAYS } & \% \\ 83 \% & \text { Canada } & 94 \% \\ 3 \% & \text { États-Unis } & 5 \% \\ 2 \% & \text { Australie } & 1 \% \\ 12 \% & & \\ & & \\ \% & \text { SECTEUR } \\ 37 \% & \text { Projets d'énergie } & 56 \% \\ 25 \% & \text { PPP/PFI } & 25 \% \\ 16 \% & \text { Projets d'infrastructure } & 12 \% \\ 9 \% & \text { Ressources Naturelles } & 5 \% \\ 8 \% & \text { Produits chimiques et gaz naturel liquéfié } & 1 \% \\ 5 \% & \text { Générique } & 1 \%\end{array}$

Les cotes de crédit de l'échantillon coté de développement et de l'échantillon coté de validation devraient être normalement distribuées autour de la cote moyenne. On peut voir dans les histogrammes suivants la répartition de la cote senior non adossée des échantillons autour d'une moyenne de BBB. Gevero (2014) et Deluz (2010) affirment que la moyenne des cotes de crédit sénior non adossées des projets de financements est de BBB-(Baa3). 
On remarque une distribution en forme de cloche (distribution normale) autour de la cote alphanumérique moyenne BBB+.

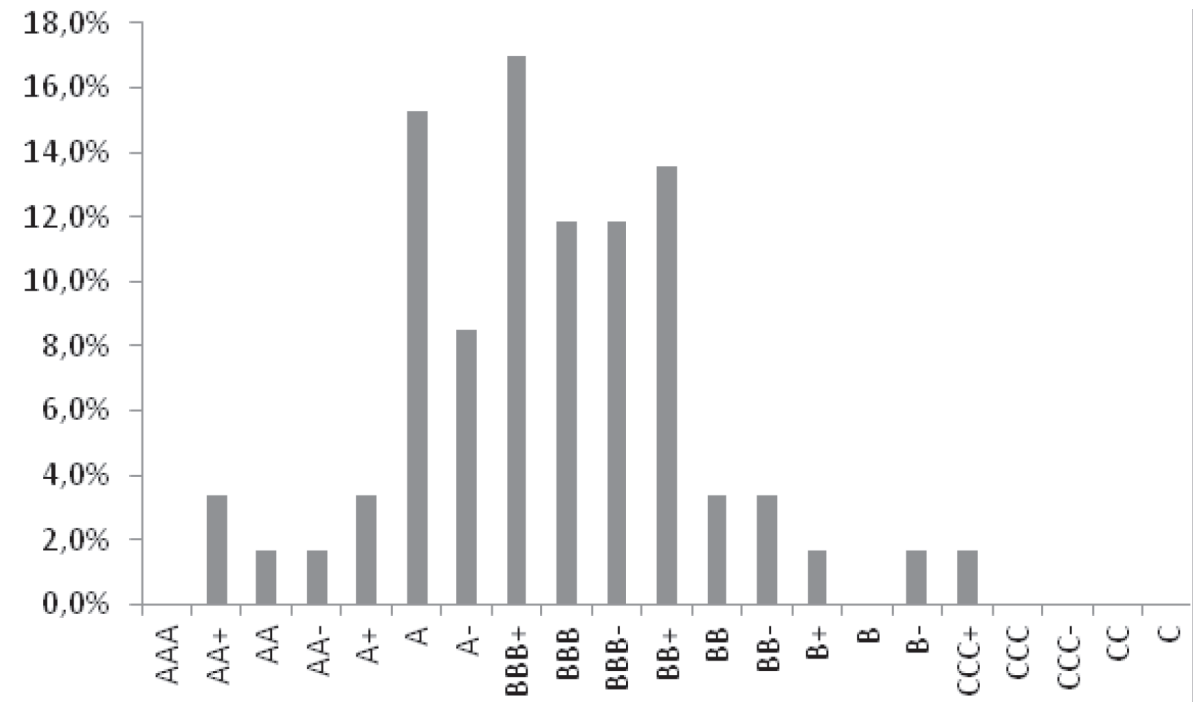

\section{FIGURE 7 Histogramme de distribution des cotes de crédit alpha de l'échantillon coté de validation}

Le même échantillon que la figure 6 est utilisé, par contre les cotes de crédit sont regroupées par lettre. Par exemple, les cotes BBB+, BBB et BBB- sont toutes regroupées dans BBB. D'où l'appellation de cote de crédit alpha au lieu de cotes de crédit alphanumérique. On remarque une distribution en forme de cloche (distribution normale) autour de la cote alpha moyenne BBB.

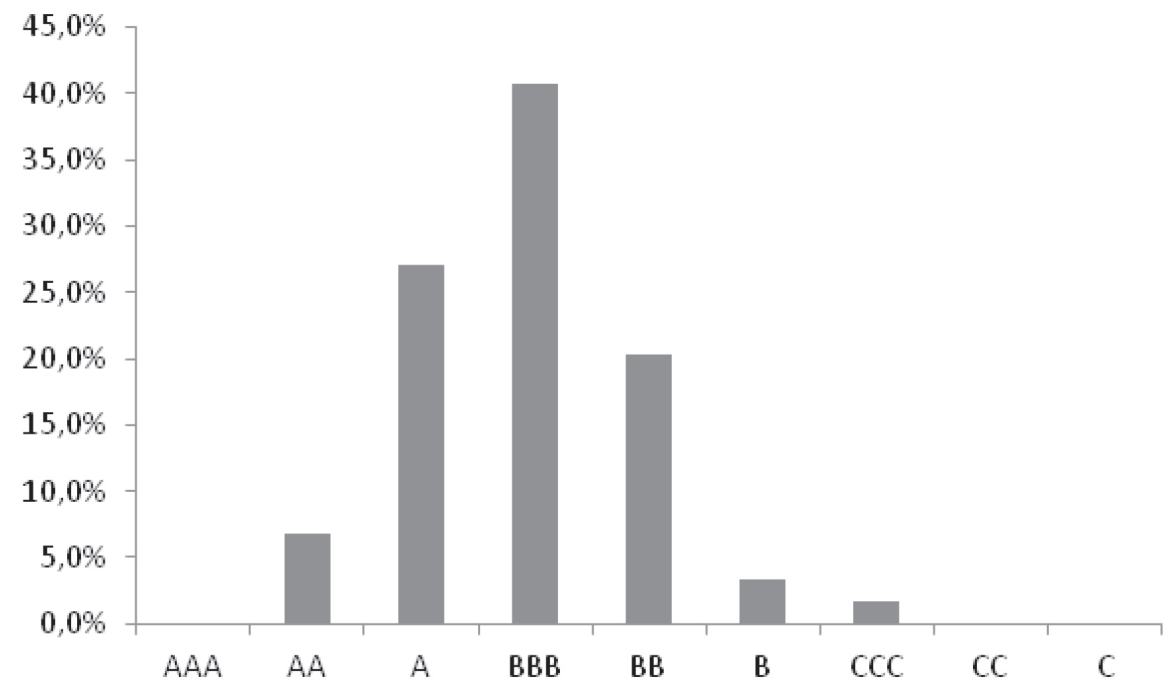


On remarque une distribution en forme de cloche (distribution normale) autour de la cote alphanumérique moyenne A-.

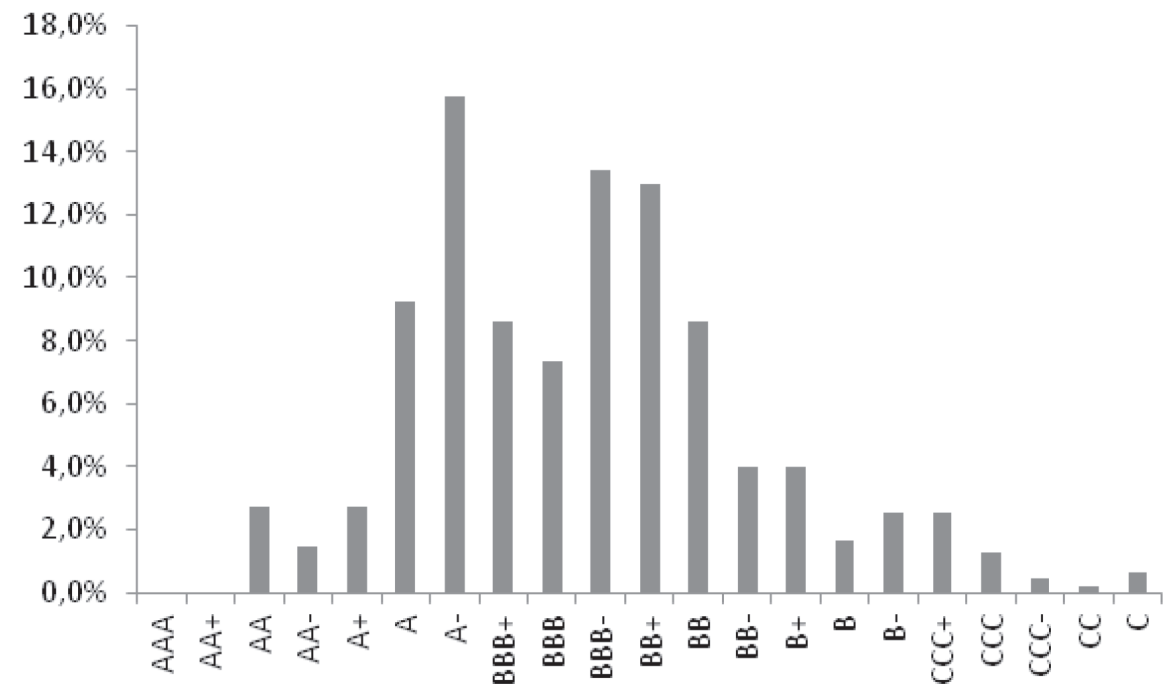

FIGURE 9 Histogramme de distribution des cotes de crédit alpha de l'échantillon coté de développement

Le même échantillon que la figure 8 est utilisé, par contre les cotes de crédit sont regroupées par lettre. Par exemple, les cotes BBB+, BBB et BBB- sont toutes regroupées dans BBB. D'où l'appellation de cote de crédit alpha au lieu de cotes de crédit alphanumériques. On remarque une distribution en forme de cloche (distribution normale) autour de la cote alpha moyenne BBB.

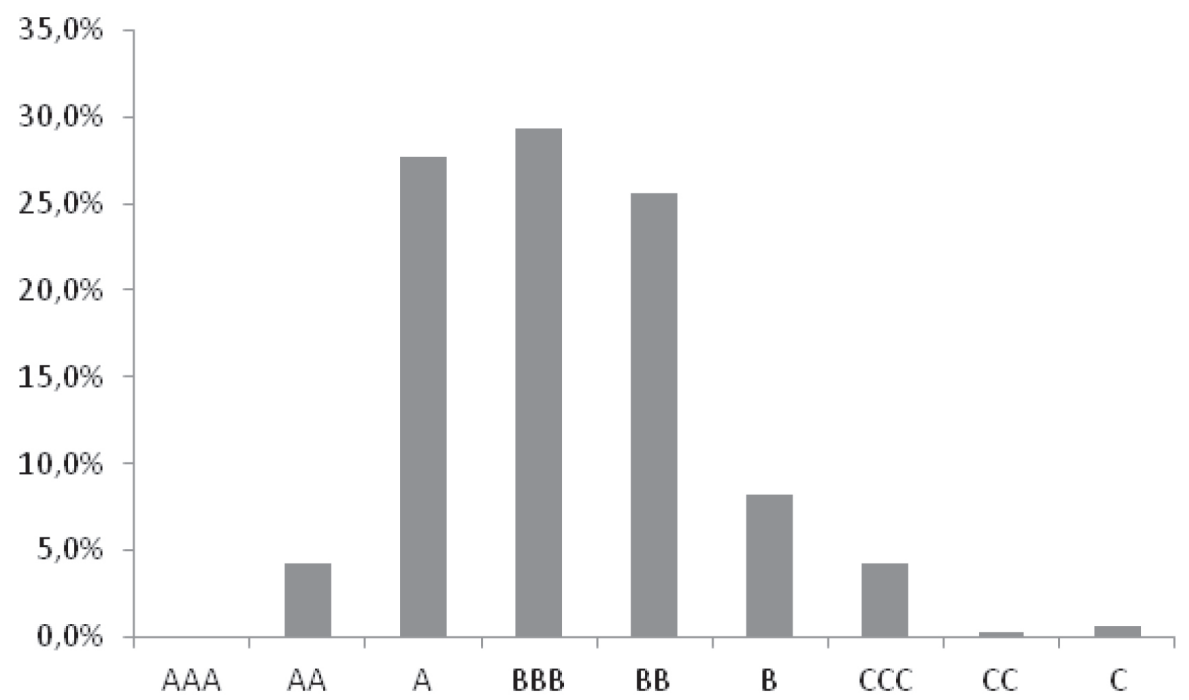


En plus des variables propres à chaque projet, des variables macroéconomiques ont également été testées. Voici donc ces variables macroéconomiques dont les séries chronologiques proviennent du site de la World Bank:
1) Taux préférentiel
2) Taux d'intérêt réel
3) Inflation basée sur le déflateur du PIB
4) Croissance du PIB

Source: http://data.worldbank.org/

Crăciun (2011) stipule que les deux variables macroéconomiques les plus importantes à considérer lors de l'appréciation du risque des projets d'infrastructure sont l'inflation et les taux d'intérêt. Ces deux facteurs macroéconomiques sont respectivement représentés par l'inflation basée sur le déflateur du PIB et le taux préférentiel. Le taux d'intérêt réel est dans la liste car il représente à la fois les taux d'intérêt et l'inflation. Finalement, Verdouw (2015) stipule que la croissance du PIB est un facteur qui influence les investissements en financement de projets. Le nombre de facteurs macroéconomique est limité à quatre car il faut garder en tête que le but de cet essai n'est pas de faire une étude économétrique de l'influence des facteurs macroéconomiques sur les probabilités de défaut des financements de projet, mais bien de développer un modèle de notation de crédit pour ce dernier.

Selon Erlenmaier (2011), la dernière étape de la construction de l'échantillon est d'analyser la structure de corrélation des facteurs. Ceci est fait à la fin de la section 6.5.4, soit l'analyse univariée des données.

\subsubsection{Données qualitatives}

Tout comme pour la sélections des facteurs quantitatifs, une revue de la documentation de notation de financement de projet de Fitch, S\&P, Moody's et DBRS est faite. Les facteurs qualitatifs d'un modèle doivent être clairement définis et encadrés afin que d'une personne à l'autre, les résultats soient les mêmes. Dans cette optique, la simplicité des définitions des facteurs et une échelle d'évaluation bien divisée sont deux éléments essentiels de bons facteurs qualitatifs. À la figure 10, une synthèse des facteurs qualitatifs retrouvés dans les documentations de notation des agences citées plus haut est faite. 
FIGURE 10 Synthèse des descriptions des facteurs qualitatifs utilisés dans les documentations méthodologiques de notation de crédit des agences de notation Fitch, S\&P, Moody's et DBRS

On y retrouve les facteurs qualitatifs et leur description respective.

FACTEUR

Risque de commanditaire

Des commanditaires expérimentés et de grande qualité de crédit réduisent la probabilité de défaut du projet. Un sponsor détenteur d'une proportion importante de l'équité totale du projet est susceptible d'y apporter son support en cas de problème. Un commanditaire peut être le parent d'une contrepartie clé impliquée dans le projet (ex: contracteur, opérateur, ...). En cas de détresse de sa filiale, le parent peut garantir une portion de ses engagements, réduisant ainsi la probabilité de défaut du projet. Ce facteur de risque mesure la qualité globale des sponsors les plus influents du projet et leur propension à apporter un support au projet.

Risque de construction

Un contracteur réputé jumelé à une technologie fiable ainsi qu'un contrat de construction de qualité supérieure présentant des protections contre les délais, dépassement de coûts et défaut de performance réduisent la probabilité de défaut du projet. Ce facteur de risque mesure la qualité globale des contracteurs/technologie/ contrat de la phase de construction du projet.

Risque d'opération $\quad$ Un opérateur réputé jumelé à une technologie fiable ainsi que l'existence de clauses de transfert de risque aux contrats d'opération réduisent la probabilité de défaut du projet. Ce facteur de risque mesure la qualité globale des opérateurs/technologie/contrats de la phase d'opération du projet.

Risque de revenu

La qualité de crédit de l'acheteur réduit la probabilité de défaut du projet. Les flux monétaires d'un projet sont exposés au risque d'inadéquations entre les revenus et les coûts qui origine de l'incertitude autour des prix et des volumes. L'incertitude autour des flux monétaires augmente la probabilité de défaut du projet. Ce facteur de risque mesure le risque des revenus du projet.

Risque souverain et politique

La qualité de l'environnement politique, réglementaire et économique sont des éléments qui réduisent la probabilité de défaut du projet au même titre que la qualité de crédit du pays hôte du projet. Ce facteur de risque mesure la qualité générale du pays et de l'environnement politique du projet. Des exemples d'événements à risque sont: un changement de régulation, un changement au niveau des taxes, une expropriation, une nationalisation, une guerre civile, une destruction d'actif, etc.

Risque d'industrie et de non-compétitivité

Risque de liquidité

Risque

de caractéristiques de financement du projet
La position compétitive d'un projet et la viabilité de l'industrie dans laquelle il œuvre réduisent la probabilité de défaut. Ce facteur mesure la compétitivité du projet visà-vis de ses pairs et la santé financière de l'industrie dans laquelle il œuvre dans la conjoncture actuelle et prospective.

Les provisions peuvent réduire la probabilité de défaut du projet à titre de sources de liquidité. Une réserve pour le service de la dette (DSR) de 6 mois est une caractéristique de liquidité standard pour un financement de projet. Ce standard peut changer pour des projets avec une forte variabilité des flux monétaires, qui eux nécessitent un niveau de DSR plus élevé. Ce facteur de risque mesure le niveau de conformité du DSR du projet face au DSR de référence («benchmark») de projets similaires.

Les financements de projet doivent respecter certains «standards » communs quant aux caractéristiques de financement d'influence sur la probabilité de défaut. Ces «standards» sont les suivants:

1) Un ensemble de mesures de sécurité qui donne accès à tous les actifs tangibles, les comptes et les revenus aux détenteurs de dette en cas de défaut du projet.

2) Un test de distribution des dividendes robuste et qui tient compte de la performance actuelle et des besoins futurs du projet.

Ce facteur mesure le niveau de conformité du projet face aux «standards » des caractéristiques de financements de projet. 
À la figure 11, les échelles d'évaluation des facteurs présentés à la figure 10 sont présentées. Les échelles des différentes agences n'avaient pas toutes le même nombre d'échelons. Pour fin de simplicité, toutes les échelles des différentes agences de notation sont ramenées sur une échelle commune à 3 échelons.

\section{FIGURE 11 Échelle d'évaluation des facteurs qualitatifs simplifiés des agences de notation Fitch, S\&P, Moody's et DBRS}

On y retrouve les facteurs qualitatifs la description de chaque échelon de leur échelle d'évaluation.

\begin{tabular}{|c|c|c|c|}
\hline \multicolumn{2}{|r|}{1} & 2 & 3 \\
\hline $\begin{array}{l}\text { Risque de } \\
\text { commanditaire }\end{array}$ & $\begin{array}{l}\text { Commanditaire avec une } \\
\text { faible expérience dans des } \\
\text { projets similaires OU } \\
\text { Qualité de crédit de type } \\
\text { spéculative (Ou les états } \\
\text { financiers ne sont pas } \\
\text { disponibles) OU } \\
\text { Engagement faible dans le } \\
\text { projet au niveau de l'équité } \\
\text { et/ou d'un support d'affaires } \\
\text { et de crédit faible (ex: entité } \\
\text { parente au contracteur/ } \\
\text { opérateur) }\end{array}$ & $\begin{array}{l}\text { Commanditaire avec une } \\
\text { expérience moyenne dans des } \\
\text { projets similaires ET } \\
\text { Qualité de crédit de type non- } \\
\text { spéculative ET } \\
\text { Engagement moyen dans le } \\
\text { projet au niveau de l'équité } \\
\text { et/ou d'un support d'affaires } \\
\text { et de crédit moyen (ex: entité } \\
\text { parente au contracteur/ } \\
\text { opérateur) }\end{array}$ & $\begin{array}{l}\text { Commanditaire avec une forte } \\
\text { expérience dans des projets } \\
\text { similaires ET } \\
\text { Qualité de crédit de type non- } \\
\text { spéculative ET } \\
\text { Engagement fort dans le projet } \\
\text { au niveau de l'équité et/ou } \\
\text { d'un support d'affaires et de } \\
\text { crédit fort (ex: entité parente } \\
\text { au contracteur/opérateur) }\end{array}$ \\
\hline $\begin{array}{l}\text { Risque de } \\
\text { construction } \\
\text { (0 si le projet } \\
\text { n'est plus en } \\
\text { construction) }\end{array}$ & $\begin{array}{l}\text { Technologie nouvelle ou } \\
\text { processus de construction } \\
\text { complexe OU } \\
\text { Contracteur avec une faible } \\
\text { expérience dans des projets } \\
\text { similaires OU } \\
\text { Qualité de crédit du } \\
\text { contracteur de type } \\
\text { spéculative OU } \\
\text { Contracteur irremplaçable OU } \\
\text { Contrat de construction } \\
\text { de qualité faible avec des } \\
\text { protections partielles ou } \\
\text { absentes contre les délais, } \\
\text { dépassements de coûts et } \\
\text { défauts de performance }\end{array}$ & $\begin{array}{l}\text { Technologie ayant fait ses } \\
\text { preuves et/ou processus de } \\
\text { construction simple ET } \\
\text { Contracteur avec une } \\
\text { expérience moyenne dans des } \\
\text { projets similaires ET } \\
\text { Qualité de crédit du } \\
\text { contracteur de type non- } \\
\text { spéculative ET } \\
\text { Contracteur remplaçable ET } \\
\text { Contrat de construction de } \\
\text { qualité moyenne avec des } \\
\text { protections raisonnables } \\
\text { contre les délais, } \\
\text { dépassements de coûts et } \\
\text { défauts de performance }\end{array}$ & $\begin{array}{l}\text { Technologie ayant fait ses } \\
\text { preuves et/ou processus de } \\
\text { construction simple ET } \\
\text { Contracteur avec une forte } \\
\text { expérience dans des projets } \\
\text { similaires ET } \\
\text { Qualité de crédit du } \\
\text { contracteur de type non- } \\
\text { spéculative ET } \\
\text { Contracteur remplaçable ET } \\
\text { Contrat de construction de } \\
\text { qualité supérieure (EPC) avec } \\
\text { des protections fortes contre } \\
\text { les délais, dépassements } \\
\text { de coûts et défauts de } \\
\text { performance }\end{array}$ \\
\hline
\end{tabular}




\begin{tabular}{|c|c|c|c|}
\hline \multicolumn{2}{|r|}{1} & 2 & 3 \\
\hline $\begin{array}{l}\text { Risque } \\
\text { d'opération }\end{array}$ & $\begin{array}{l}\text { Technologie nouvelle ou } \\
\text { processus d'opération } \\
\text { complexe qui résulte } \\
\text { en des caractéristiques } \\
\text { d'opération et de performance } \\
\text { incertaines OU } \\
\text { Opérateur avec une faible } \\
\text { expérience dans des projets } \\
\text { similaires OU } \\
\text { Qualité de crédit de } \\
\text { l'opérateur de type } \\
\text { spéculative OU } \\
\text { Opérateur irremplaçable OU } \\
\text { Contrat d'opération de qualité } \\
\text { faible avec des protections } \\
\text { partielles/absentes sur les } \\
\text { paramètres clés de la phase } \\
\text { d'opération }\end{array}$ & $\begin{array}{l}\text { Technologie ayant fait ses } \\
\text { preuves et/ou processus } \\
\text { d'opération moyennement } \\
\text { complexe qui résulte } \\
\text { en des caractéristiques } \\
\text { d'opération et de performance } \\
\text { moyennement prévisibles ET } \\
\text { Opérateur avec une } \\
\text { expérience moyenne dans des } \\
\text { projets similaires ET } \\
\text { Qualité de crédit de } \\
\text { l'opérateur de type non- } \\
\text { spéculative ET } \\
\text { Opérateur remplaçable ET } \\
\text { Contrat d'opération de } \\
\text { qualité moyenne avec des } \\
\text { protections raisonnables sur } \\
\text { les paramètres clés de la } \\
\text { phase d'opération }\end{array}$ & $\begin{array}{l}\text { Technologie ayant fait ses } \\
\text { preuves et/ou processus } \\
\text { d'opération simple qui résulte } \\
\text { en des caractéristiques } \\
\text { d'opération et de performance } \\
\text { fortement prévisibles ET } \\
\text { Opérateur avec une forte } \\
\text { expérience dans des projets } \\
\text { similaires ET } \\
\text { Qualité de crédit de l'opérateur } \\
\text { de type non- spéculative ET } \\
\text { Opérateur remplaçable ET } \\
\text { Contrat d'opération de } \\
\text { qualité supérieure avec des } \\
\text { protections fortes sur les } \\
\text { paramètres clés de la phase } \\
\text { d'opération }\end{array}$ \\
\hline Risque de revenu & $\begin{array}{l}\text { Qualité de crédit de l'acheteur } \\
\text { de type spéculative OU } \\
\text { Acheteur irremplaçable OU } \\
\text { Revenus faiblement } \\
\text { prévisibles OU } \\
\text { Inadéquations (revenus vs } \\
\text { coûts) substantielles sur } \\
\text { de longues périodes }\end{array}$ & $\begin{array}{l}\text { Notation à long terme de } \\
\text { l'acheteur }\{\text { Corporatif } \\
\text { [BBB-, } A+]\} \text { ET } \\
\text { Acheteur remplaçable ET } \\
\text { Revenus moyennement } \\
\text { prévisibles ET } \\
\text { Inadéquations (revenus vs } \\
\text { coûts) mineures et de très } \\
\text { courtes durées }\end{array}$ & $\begin{array}{l}\text { Notation à long terme } \\
\text { de l'acheteur \{Corporatif } \\
\text { [A+, AAA], Souverain/ sous- } \\
\text { souverain de type } \\
\text { non-spéculatif\} ET } \\
\text { Acheteur remplaçable ET } \\
\text { Revenus fortement } \\
\text { prévisibles ET } \\
\text { Inadéquations (revenus } \\
\text { vs coûts) absentes ou } \\
\text { très mineures }\end{array}$ \\
\hline $\begin{array}{l}\text { Risque souverain } \\
\text { et politique }\end{array}$ & $\begin{array}{l}\text { Notation à long terme du } \\
\text { pays hôte du projet de type } \\
\text { spéculatif OU } \\
\text { Juridiction, stabilité politique } \\
\text { et économique non fiable }\end{array}$ & $\begin{array}{l}\text { Notation à long terme du pays } \\
\text { hôte du projet [BBB-, } A+] \text { ET } \\
\text { Juridiction, stabilité politique } \\
\text { et économique fiable }\end{array}$ & $\begin{array}{l}\text { Notation à long terme du pays } \\
\text { hôte du projet }[A+, A A A] \text { ET } \\
\text { Juridiction, stabilité politique } \\
\text { et économique très fiable }\end{array}$ \\
\hline $\begin{array}{l}\text { Risque } \\
\text { d'industrie } \\
\text { et de non- } \\
\text { compétitivité }\end{array}$ & $\begin{array}{l}\text { Avantage compétitif faible/ } \\
\text { absent OU } \\
\text { Industrie nouvelle, } \\
\text { perspectives anticipées } \\
\text { négatives }\end{array}$ & $\begin{array}{l}\text { Avantage compétitif moyen, } \\
\text { protégé par des barrières } \\
\text { à l'entrée ET } \\
\text { Industrie établie, perspectives } \\
\text { anticipées stables }\end{array}$ & $\begin{array}{l}\text { Situation de monopole/ } \\
\text { avantage compétitif fort } \\
\text { protégé par des barrières } \\
\text { à l'entrée significatives ET } \\
\text { Industrie établie, perspectives } \\
\text { anticipées positives }\end{array}$ \\
\hline $\begin{array}{l}\text { Risque } \\
\text { de liquidité }\end{array}$ & $\begin{array}{l}\text { DSR du projet est } \\
\text { significativement inférieure } \\
\text { à la DSR de référence de } \\
\text { projets similaires }\end{array}$ & $\begin{array}{l}\text { DSR du projet est conforme } \\
\text { à la DSR de référence } \\
\text { de projets similaires }\end{array}$ & $\begin{array}{l}\text { DSR du projet est } \\
\text { significativement supérieure } \\
\text { à la DSR de référence } \\
\text { de projets similaires }\end{array}$ \\
\hline $\begin{array}{l}\text { Risque de } \\
\text { caractéristiques } \\
\text { de financement } \\
\text { du projet }\end{array}$ & $\begin{array}{l}\text { Les caractéristiques de } \\
\text { financement du projet sous- } \\
\text { performent les «standards» } \\
\text { énumérés }\end{array}$ & $\begin{array}{l}\text { Les caractéristiques de } \\
\text { financement du projet sont } \\
\text { conformes aux «standards» } \\
\text { énumérés }\end{array}$ & $\begin{array}{l}\text { Les caractéristiques de } \\
\text { financement du projet } \\
\text { surperforment les «standards» } \\
\text { énumérés }\end{array}$ \\
\hline
\end{tabular}


Afin de collecter les données, un gabarit semblable à celui des facteurs qualitatifs est créé. Le gabarit est ensuite rempli avec les définitions et les échelles présentées aux figures 10 et 11. Un exemple du gabarit rempli est présenté dans le tableau 12.

TABLEAU 12 Exemple d'une ligne du gabarit des facteurs qualitatifs rempli avec les définitions de la figure 10 et les échelles de la figure 11

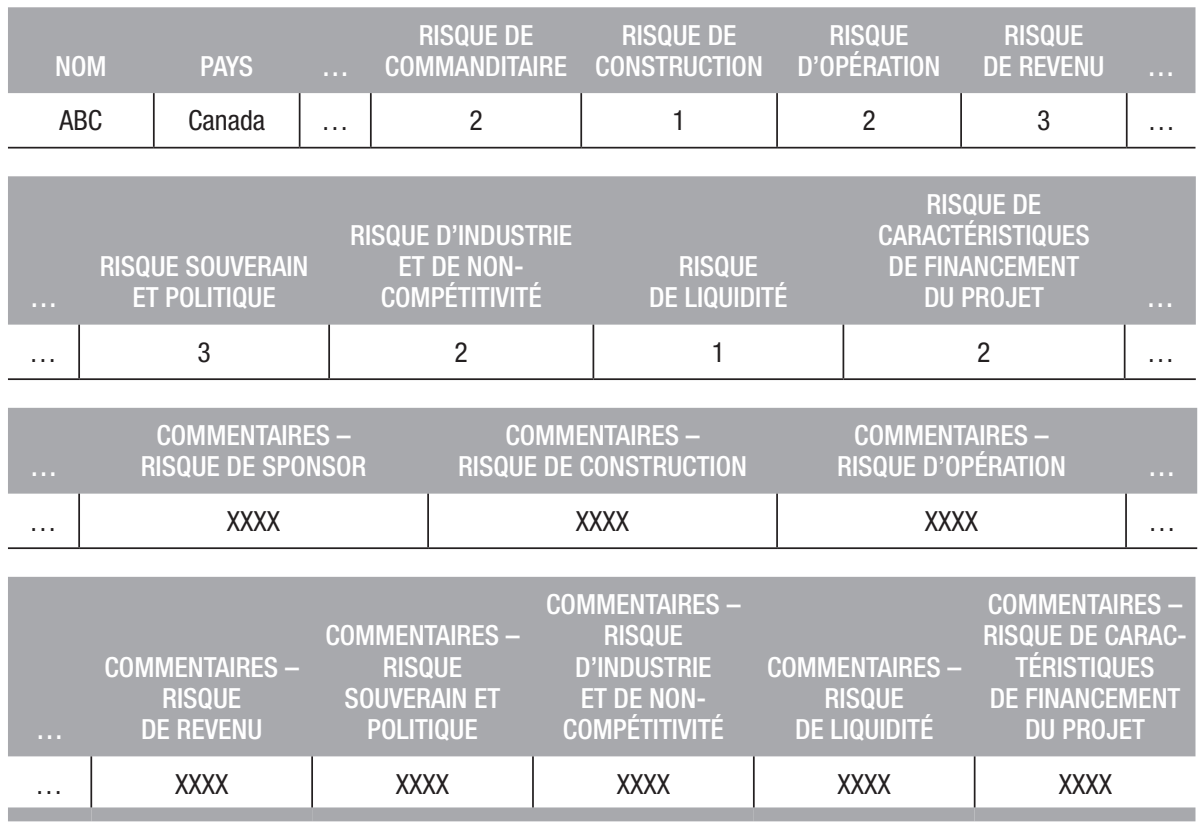

Après avoir rempli le gabarit, nous nous retrouvons avec l'échantillon de développement du modèle qualitatif et quantitatif qui a les caractéristiques suivantes :

\section{TABLEAU 13 Nombre de contreparties et de contreparties-année dans l'échantillon de développement du modèle qualitatif et quantitatif par pays}

Une contrepartie-année est équivalent à un état financier pour un financement de projet. La majorité des contreparties et des contreparties-année sont au Canada.

\begin{tabular}{l|c|c}
\multicolumn{1}{c|}{ PAYS } & NOMBRE DE CONTREPARTIES & NOMBRE DE CONTREPARTIE-ANNÉE \\
Canada & 27 & 86 \\
\hline Mexico & 2 & 6
\end{tabular}


TABLEAU 14 Nombre de contreparties et de contreparties-année dans l'échantillon de développement du modèle qualitatif et quantitatif par secteur

Une contrepartie-année est équivalente à un état financier pour un financement de projet. Les secteurs des produits chimiques et gaz naturel liquéfié, des ressources naturelles et génériques ne sont pas présents dans l'échantillon. Seulement les secteurs de l'énergie, de l'infrastructure et des PPP/PFI sont présents. Les trois secteurs présents sont environ représentés à proportion égales dans l'échantillon.

\begin{tabular}{|c|c|c|}
\hline SECTEUR & NOMBRE DE CONTREPARTIES & NOMBRE DE CONTREPARTIE-ANNÉE \\
\hline Énergie & 9 & 34 \\
\hline Infrastructure & 7 & 31 \\
\hline PPP/PFI & 13 & 27 \\
\hline
\end{tabular}

On remarque que l'échantillon est principalement composé de projets canadiens. De plus, seulement trois secteurs sur six sont représentés dans l'échantillon. Par contre, il y a une bonne répartition des projets entre les différents secteurs. Le score de 1 à 3 donné à chaque facteur quantitatif pour chaque projet est considéré constant dans le temps pour le développement du modèle.

Avant d'aller plus loin dans le développement du modèle, les histogrammes de distributions des facteurs qualitatifs de l'échantillon sont observés. Les histogrammes sont présentés dans les figures 12 à 19 ci-dessous. On remarque que la plupart des facteurs n'ont que des observations dans deux des trois scores possibles. Une autre observation est que la plupart des facteurs ont une très forte concentration dans un seul score. Il était connu que l'analyse purement économétrique allait être difficile pour la partie qualitative du modèle dû au petit nombre d'observations disponibles, mais les deux points mentionnés ci-haut la rendent pratiquement impossible. 
FIGURE 12 Histogramme de distribution dans l'échelle du facteur qualitatif «Risque de commanditaire» présenté dans la figure 11

L'échelon 1 représente le pire score de l'échelle d'un point de vue de qualité de crédit et l'échelon 3 le meilleur. II y a des observations dans les trois échelons de l'échelle.

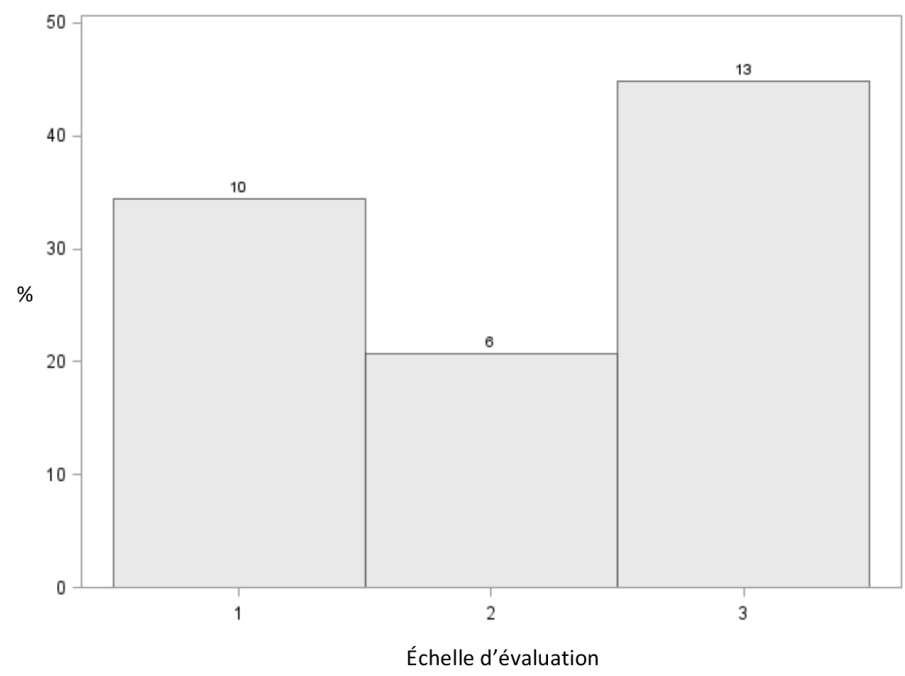

FIGURE 13 Histogramme de distribution dans l'échelle du facteur qualitatif «Risque de construction» présenté dans la figure 11

L'échelon 1 représente le pire score de l'échelle d'un point de vue de qualité de crédit et l'échelon 3 le meilleur. L'échelon 4 représente les projets qui ne sont plus en construction. II n'y a pas d'observations dans les quatre échelons de l'échelle et la majorité des projets ne sont plus en construction.

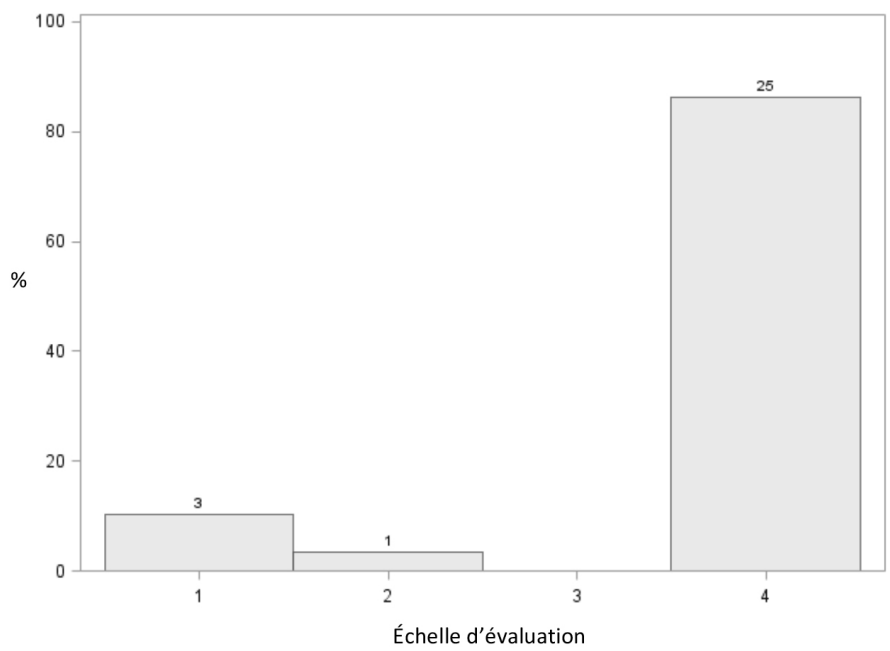




\section{FIGURE 14 Histogramme de distribution dans l'échelle du facteur qualitatif «Risque d'opération» présenté dans la figure 11}

L'échelon 1 représente le pire score de l'échelle d'un point de vue de qualité de crédit et l'échelon 3 le meilleur. La majorité des observations sont dans l'échelon 3 de l'échelle. Les projets ont donc tous un risque d'opération faible.

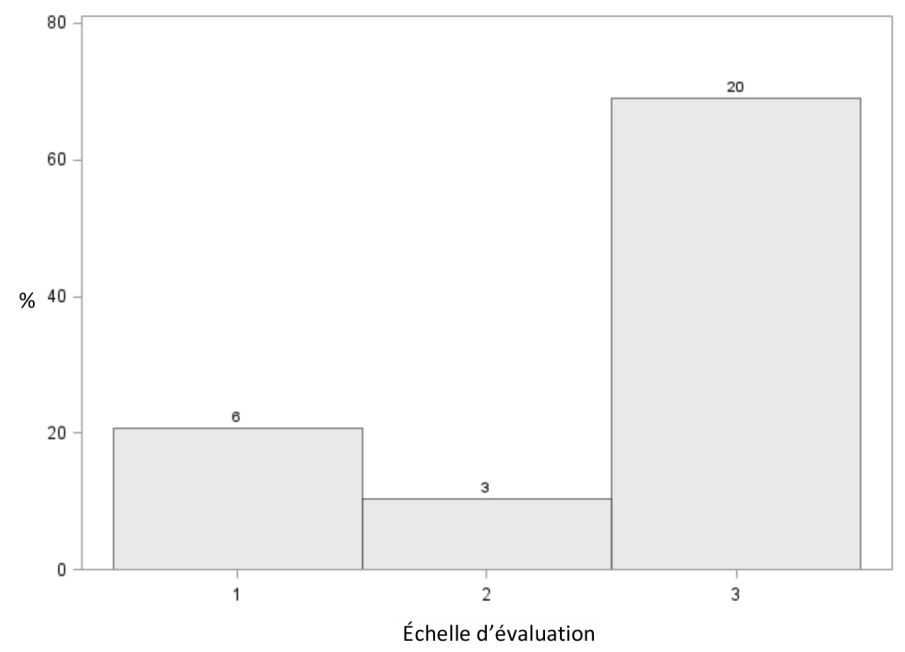

\section{FIGURE 15 Histogramme de distribution dans l'échelle du facteur qualitatif «Risque de revenu » présenté dans la figure 11}

L'échelon 1 représente le pire score de l'échelle d'un point de vue de qualité de crédit et l'échelon 3 le meilleur. II y a des observations dans les trois échelons de l'échelle. Par contre, une forte majorité des observations se trouve dans les deux échelons les plus forts de l'échelle. Près de $80 \%$ des projets ont un risque de revenu de modéré à faible.

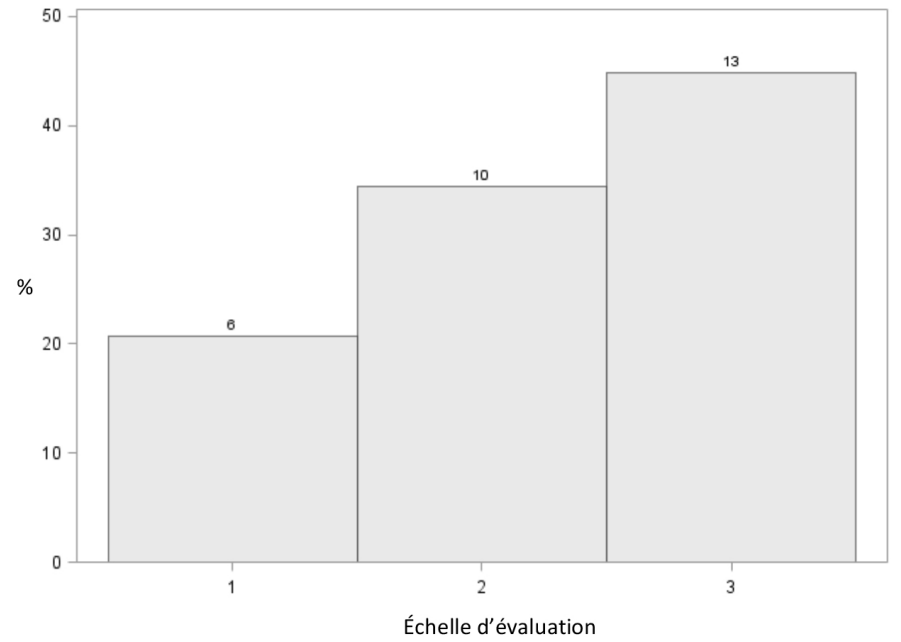


FIGURE 16 Histogramme de distribution dans l'échelle du facteur qualitatif «Risque souverain et politique» présenté dans la figure 11

L'échelon 1 représente le pire score de l'échelle d'un point de vue de qualité de crédit et l'échelon 3 le meilleur. II n'y a pas d'observations dans les trois échelons de l'échelle et plus de $93 \%$ des projets ont un risque souverain et politique faible.

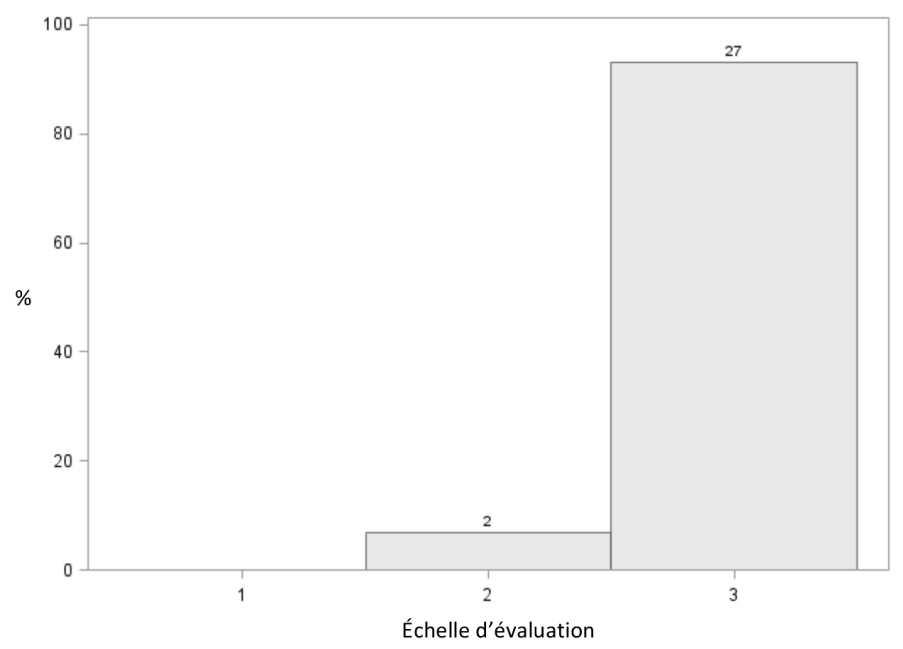

\section{FIGURE 17 Histogramme de distribution dans l'échelle du facteur qualitatif «Risque d'industrie et de non-compétitivité» présenté dans la figure 11}

L'échelon 1 représente le pire score de l'échelle d'un point de vue de qualité de crédit et l'échelon 3 le meilleur. II n'y a pas d'observations dans les trois échelons de l'échelle et plus de $85 \%$ des projets ont un risque d'industrie et de non-compétitivité faible.

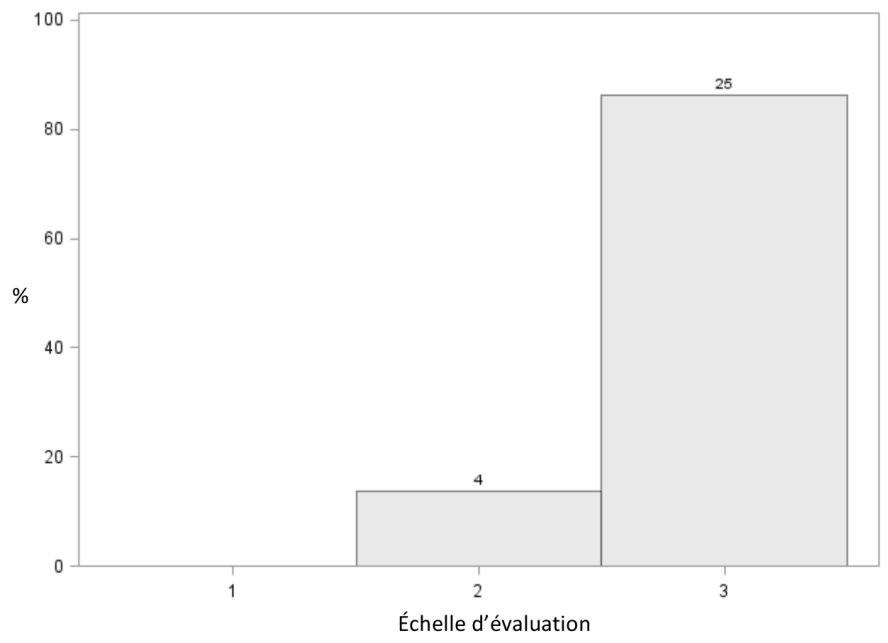


FIGURE 18 Histogramme de distribution dans l'échelle du facteur qualitatif «Risque de liquidité» présenté dans la figure 11

L'échelon 1 représente le pire score de l'échelle d'un point de vue de qualité de crédit et l'échelon 3 le meilleur. II n'y a pas d'observations dans les trois échelons de l'échelle et près de $83 \%$ des projets ont un risque de liquidité modéré.

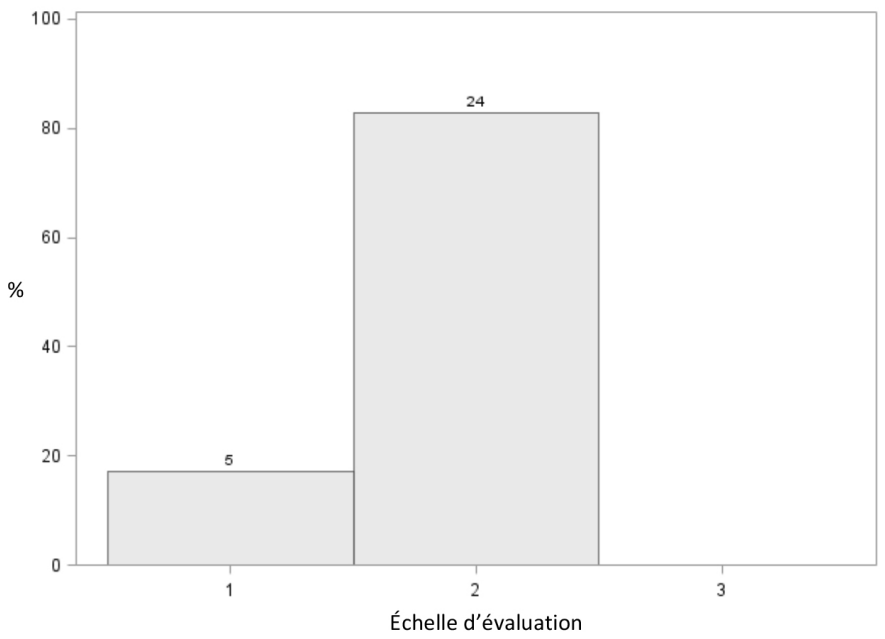

FIGURE 19 Histogramme de distribution dans l'échelle du facteur qualitatif «Risque de caractéristiques de financement du projet» présenté dans la figure 11

L'échelon 1 représente le pire score de l'échelle d'un point de vue de qualité de crédit et l'échelon 3 le meilleur. II y a des observations dans les 3 échelons de l'échelle. La majorité des projets ont un risque de caractéristiques de financement modéré.

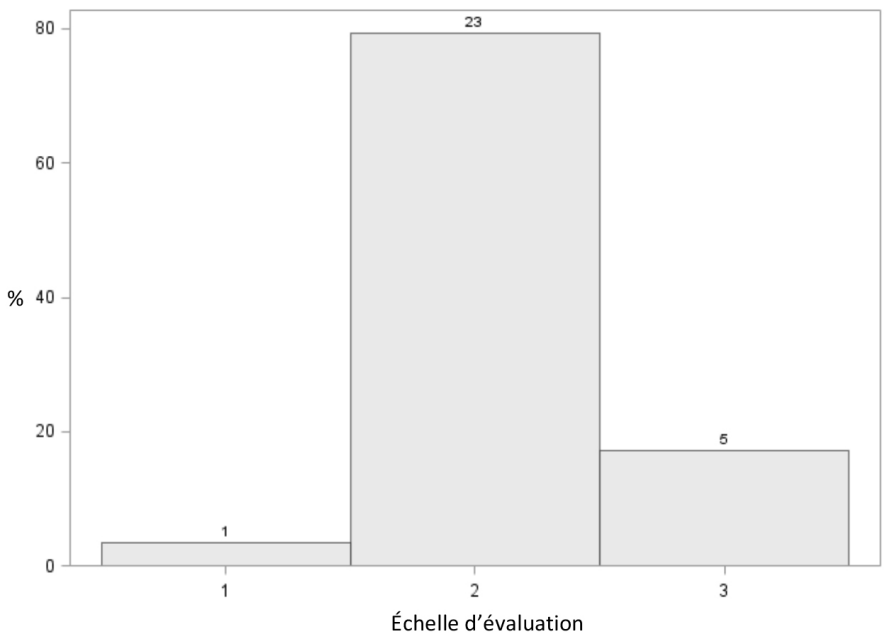




\subsection{Traitement des données}

Dans cette section, les traitements qui sont appliqués aux données sont présentés dans l'ordre suivant: ceux appliqués sur les cotes de crédit externes; ceux appliqués sur les variables financières propres à chaque projet; ceux appliqués sur les variables macroéconomiques : puis, ceux appliqués sur les facteurs qualitatifs.

\subsubsection{Traitement des cotes de crédit}

Grâce aux tableaux 1 et 2, chacune des cotes des agences de notation externes de différentes séniorités peuvent être ramenées à la cote de l'émetteur et à une échelle commune de 1 à 21 . Une fois chacune des cotes ramenées sur cette échelle, la moyenne des cotes numériques est calculée. Cette moyenne est ensuite arrondie à l'entier le plus proche et retransformée sur l'échelle alpha numérique de Moody's. De cette façon, chacune des années/projet n'a qu'une seule cote de crédit. Cette cote unique est par la suite transformée en probabilité de défaut (PD) avec la table de transition (Tableau 3) présentée plus tôt. On arrive donc ultimement à une PD par année/projet.

\subsubsection{Traitement des données financières}

Après avoir traité les cotes de crédit, il faut maintenant traiter les données financières de chaque année/projet. La première étape consiste à calculer les ratios financiers désirés. Une fois ces ratios calculés, les six déclinaisons suivantes sont calculées pour chacun des facteurs financiers à tester:

Moyenne du ratio sur une période maximale de trois ans:

$$
\text { Moy_3Y } r_{\text {Ratio }=r, \text { Année }=j}=\sum_{i=0}^{2} \frac{\text { Ratio }_{r, j-i} * \mathbf{I}_{j-i}}{\left(\mathrm{I}_{j}+\mathrm{I}_{j-1}+\mathbf{I}_{j-2}\right)}
$$

où

Moy_3Y $r_{r, j}=$ Moyenne du ratio $r$ à l'année $j$ sur une période maximale de trois ans

$\mathrm{I}_{j}=$ Variable indicatrice qui est égale à 1 si l'année $j$ est disponible et 0 sinon

Ratio $_{r, j}=$ Ratio financier $r$ de l'année $j$. 
Maximum du ratio sur une période maximale de trois ans:

Si les trois années sont disponibles,

$$
\text { Max_3Y } r_{\text {Ratio=r } r \text { Année }=j}=\max \left(\text { Ratio }_{r, j}, \text { Ratio }_{r, j-1}, \text { Ratio }_{r, j-2}\right)
$$

Si seulement deux années sont disponibles,

$$
\text { Max_3Y } r_{\text {Ratio }=r, \text { Année }=j}=\max \left(\text { Ratio }_{r, j}, \text { Ratio }_{r, j-1}\right)
$$

Si seulement une année est disponible,

$$
\text { Max_3Y } r_{\text {Ratio }=r, \text { Année }=j}=\text { Ratio }_{r, j}
$$

où

Max_3Y $r_{r, j}=$ Maximum du ratio $r$ à l'année $j$ sur une période maximale de trois ans

Ratio $_{r, j}=$ Ratio financier $r$ de l'année $j$.

Minimum du ratio sur une période maximale de trois ans:

Si les trois années sont disponibles,

$$
\text { Min_3Y } r_{\text {Ratio } r, \text { Année }=j}=\min \left(\text { Ratio }_{r, j}, \text { Ratio }_{r, j-1}, \text { Ratio }_{r, j-2}\right)
$$

Si seulement deux années sont disponibles,

$$
\text { Min_3Y } r_{\text {Ratio }=r, \text { Année }=j}=\min \left(\text { Ratio }_{r, j}, \text { Ratio }_{r, j-1}\right)
$$

Si seulement une année est disponible,

$$
\text { Min_3Y } r_{\text {Ratio }=r, \text { Année }=j}=\text { Ratio }_{r, j}
$$

où

Min $\_3 Y_{r, j}=$ Minimum du ratio $r$ à l'année $j$ sur une période maximale de trois ans

Ratio $_{r, j}=$ Ratio financier $r$ de l'année $j$.

Moyenne du logarithme du ratio sur une période maximale de trois ans (les ratios négatifs sont exclus du test):

$$
\text { Moy_Log_3Y } r_{\text {Ratio }=r, \text { Année }=j}=\sum_{i=0}^{2} \frac{\log \left(\text { Ratio }_{r, j-i}\right) * \mathrm{I}_{j-i}}{\left(\mathrm{I}_{j}+\mathrm{I}_{j-1}+\mathrm{I}_{j-2}\right)}
$$

où

Moy_Log_3Y $r_{r, j}=$ Moyenne du logarithme du ratio $r$ de l'année $j$ sur une période maximale de trois ans 
$\mathrm{I}_{j}=$ Variable indicatrice qui est égale à 1 si l'année $j$ est disponible et 0 sinon

Ratio $_{r, j}=$ Ratio financier $r$ de l'année $j$.

Moyenne du log-rendement sur une période maximale de deux ans:

Si les deux dernières années sont disponibles

Moy_Log_Rnd_3Y $r_{\text {Ratio }=r, \text { Année }=j}=\sum_{i=0}^{1} \frac{\log \left(\frac{\text { Ratio }_{r, j-i}}{\operatorname{Ratio}_{r, j-i-1}}\right) *\left(\mathbf{I}_{j-i} * \mathbf{I}_{j-i-1}\right)}{\left(\mathbf{I}_{j} * \mathbf{I}_{j-1}+\mathbf{I}_{j-1} * \mathbf{I}_{j-2}\right)}$ où

Moy_Log_Rnd_3Y $r_{r, j}=$ Moyenne du log-rendement du ratio $r$ de l'année $j$ sur une période maximale de deux ans

$\mathrm{I}_{j}=$ Variable indicatrice qui est égale à 1 si l'année $j$ est disponible et 0 sinon

Ratio $_{r, j}=$ Ratio financier $r$ de l'année $j$.

S'il y a seulement une année de disponible, la moyenne du secteur de la Moyenne du log-rendement sur une période maximale de deux ans est utilisée.

Moyenne du log-rendement au carré sur une période maximale de deux ans:

Si les deux dernières années sont disponibles

Moy_Log_Sqr_Rnd_3Y $r_{\text {Ratio }=r, \text { Année }=j}=\sum_{i=0}^{1} \frac{\log \left(\frac{\text { Ratio }_{r, j-i}}{\text { Ratio }_{r, j-i-1}}\right)^{2} *\left(\mathbf{I}_{j-i} * \mathbf{I}_{j-i-1}\right)}{\left(\mathbf{I}_{j} * \mathbf{I}_{j-1}+\mathbf{I}_{j-1} * \mathbf{I}_{j-2}\right)}$

où

Moy_Log_Sqr_Rnd_3Y $r_{r, j}=$ Moyenne du log-rendement au carré du ratio $r$ à l'année $j$ sur une période maximale de deux ans

$\mathrm{I}_{j}=$ Variable indicatrice qui est égale à 1 si l'année $j$ est disponible et 0 sinon

Ratio $_{r, j}=$ Ratio financier $r$ de l'année $j$ 
S'il y a seulement une année de disponible, la moyenne du secteur de la Moyenne du log-rendement au carré sur une période maximale de deux ans est utilisée.

Une fois les déclinaisons des ratios financiers calculées, ces dernières sont transformées pour les raisons suivantes (Rabobank, 2010):

- Les valeurs aberrantes dans les données peuvent avoir une grande influence injustifiée sur les résultats de la régression. L’application d'une transformation sur les facteurs avant la régression peut diminuer leur effet.

- Grâce à la transformation, tous les facteurs peuvent avoir le même domaine. Lorsque la régression est effectuée sur ces facteurs transformés, les pondérations de facteurs qui en résultent peuvent être comparées de manière plus intuitive.

- Certains facteurs ont d'abord une répartition du score qui est inversement proportionnelle à la solvabilité. Donc, pour ces facteurs, un score plus élevé correspond à une solvabilité plus faible. Le modèle final doit être intuitif: les scores de modèle supérieur doivent appartenir à des contreparties avec une meilleure solvabilité. Pour être en mesure d'observer ceci, tous les facteurs doivent être liés positivement à la solvabilité. Par conséquent, une transformation est nécessaire pour les facteurs qui sont inversement proportionnels à la solvabilité.

Ce sont ces facteurs transformés qui seront ultimement testés dans le modèle et une transformation logistique est utilisée. Le but de cette transformation est de ramener tous les facteurs sur le même domaine commun $[0,1]$. La formule de cette transformation est la suivante:

$$
f(x)=\frac{1}{\left(1+e \frac{\mu-x}{\sigma}\right)}
$$

où $x$ est le facteur non transformé, $\mu$ est la moyenne de la distribution logistique et $\sigma$ est l'écart-type de la distribution logistique. Dans la figure 20 , on peut voir une représentation graphique de la fonction de distribution logistique avec $\mu=0$ et $\sigma=1$. 
FIGURE 20 Graphique de fonction de répartition de la fonction de distribution logistique avec les paramètres $\mu=0$ et $\sigma=1$

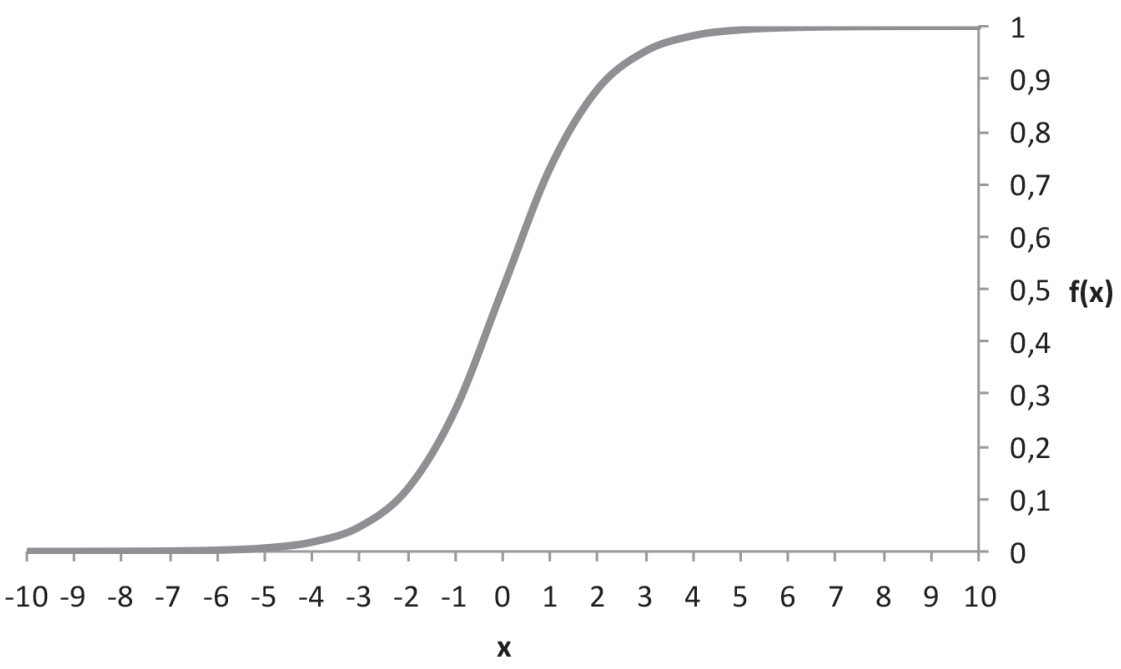

\subsubsection{Transformation logistique}

Les paramètres $a$ et $b$ ont été évalués de façon à ce que la fonction de distribution résultante de la logistique ait le meilleur ajustement avec la fonction de distribution empirique. L'estimation des paramètres est faite en excluant 5 pourcent des facteurs ayant les plus grandes et plus petites valeurs, pour un total de 10 pourcent, afin de minimiser l'effet des valeurs extrêmes. Les paramètres sont obtenus grâce à la procédure SAS LOGISTIC. Si le facteur a une relation positive avec les PD, la transformation $1-f(x)$ est appliquée. On peut voir à la figure 21 un exemple d'une transformation logistique du facteur Moyenne du ratio sur une période maximale de trois ans du service de couverture de la dette. La courbe bleue représente la distribution empirique et la courbe rouge la fonction de distribution de la loi logistique ajustée. 
FIGURE 21 Graphique des fonctions de répartition du facteur "Moyenne du ratio sur une période maximale de trois ans du service de couverture de la dette»

La fonction de répartition empirique du facteur est en bleu et la fonction de répartition logistique ajustée aux données du facteur est en rouge. Les paramètres $\mu=1,45867402$ et $\sigma=0,52746222$ sont obtenus grâce à la procédure SAS LOGISTIC.

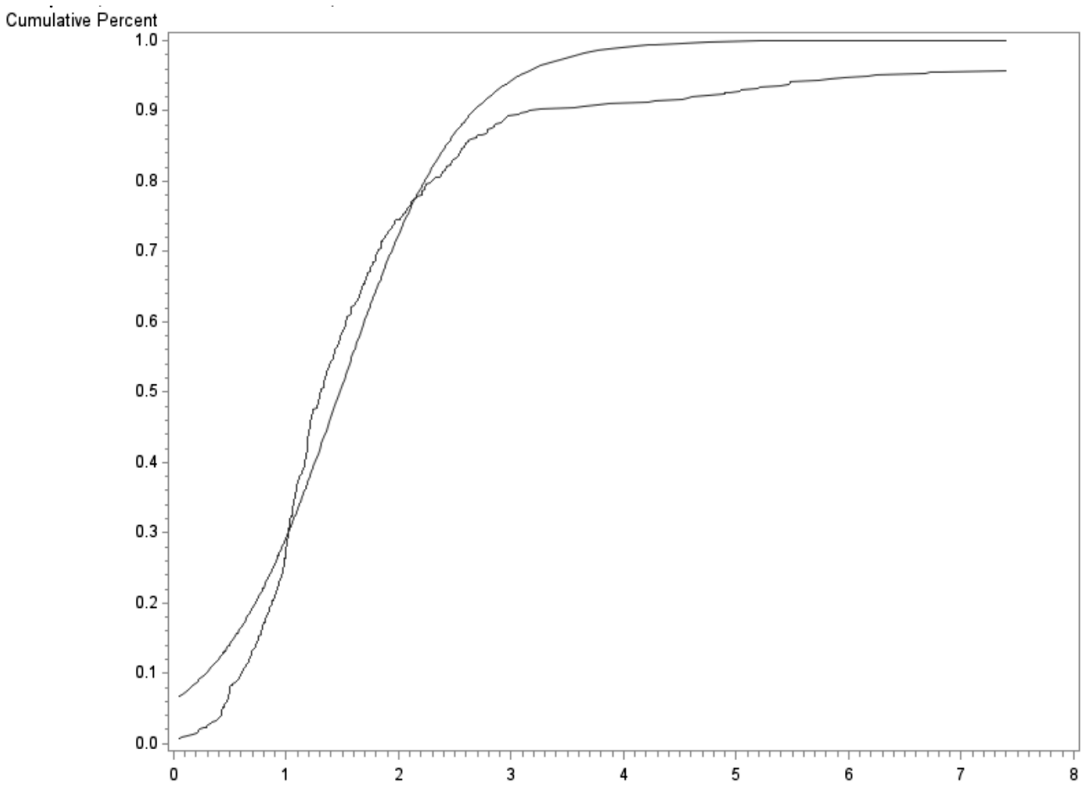

Moyenne du ratio sur une période maximale de trois ans du service de couverture de la dette

Afin de ne pas trop alourdir le texte, les noms utilisés dans le texte pour parler des facteurs transformés sont présenté dans le tableau suivant:

TABLEAU 15 Noms des facteurs complets avant transformation logistique et noms abrégés des facteurs après transformation logistique afin de ne pas alourdir le texte

\section{NOM DU FACTEUR NON TRANSFORMÉ}

Moyenne du ratio sur une période maximale de trois ans

Maximum du ratio sur une période maximale de trois ans

Minimum du ratio sur une période maximale de trois ans

Moyenne du logarithme du ratio sur une période maximale de trois ans

Moyenne du log-rendement sur une période maximale de deux ans

Moyenne du log-rendement au carré sur une période maximale de deux ans
NOM DU FACTEUR APRÈS TRANSFORMATION LOGISTIQUE

Moyenne

Maximum

Minimum

Moyenne du logarithme

Moyenne du log-rendement

Moyenne du log-rendement au carré 


\subsubsection{Test de segmentation}

Afin de voir s'il y a un effet industrie sur les ratios financiers avant de les transformer, les régressions suivantes sont effectuées:

$$
\text { Facteur }_{i}=\alpha_{\text {Secteur }, i}+\beta_{\text {Secteur }, i} \mathrm{I}_{\text {Secteur }, i}+\varepsilon_{\text {Secteur }, i}
$$

et

$$
\log \left(\mathrm{PD}_{j}\right)=\alpha_{i}+\beta_{i} \text { Facteur }_{i, j}+\varepsilon_{i}
$$

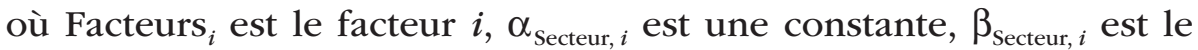
coefficient de régression, $\mathrm{I}_{\mathrm{Secteur}, i} \mathrm{une}$ variable indicatrice qui est égale à 1 si le secteur de l'observation $i$ est le secteur testé et 0 sinon, $\mathrm{PD}_{j}$ est la probabilité de défaut du financement de projet $j, \alpha_{i}$ est une constante et $\beta_{i}$ est le coefficient de régression. Cela est fait pour tous les secteurs et pour tous les facteurs. Seulement les moyennes sont testées. Les autres déclinaisons du facteur subissent le même traitement que celui sélectionné pour la moyenne.

On considère qu'un facteur ne nécessite pas de transformation logistique par secteur si les trois conditions suivantes sont respectées:

1) L'un de ses $\beta_{\text {Secteur, } i}$ est non significatif à $10 \%^{2}$

2) Son $\beta_{i}$ est significatif à $10 \%$

3) Son SAR sur une base individuel est supérieur à 0,3.

Les trois conditions précédentes sont motivées comme suit:

- La non-significativité d'un des $\beta$ des variables indicatrices de secteur donne un bon indice sur si le secteur influence le niveau du facteur.

- La significativité du $\beta$ du facteur sans variable indicatrice montre que ce dernier a un pouvoir explicatif tous secteurs confondus.

- Le SAR individuel donne un bon indice sur le pouvoir discriminant du facteur sur les probabilités de défaut.

Ces trois conditions réunies donnent un bon indice sur le pouvoir discriminant du facteur sans faire de distinction pour le secteur.

Les secteurs Énergie et PPP et infra sont testés. Il n'y a pas assez de données dans les autres secteurs pour faire des tests économétriques. Suite à cette analyse, les seuls facteurs qui ne doivent pas subir une transformation logistique par secteur sont la marge EBITDA et la marge EBIT. 
Les résultats du tableau sont obtenus en faisant les régressions : Facteurs ${ }_{i}=\alpha_{\text {Secteur }, i}+\beta_{\text {Secteur }, i} I_{\text {Secteur }, i}+\varepsilon_{\text {Secteur, }, i}$

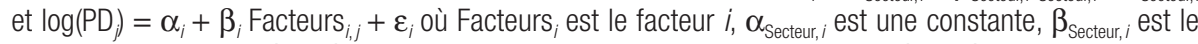
coefficient de la première régression, $I_{\text {Secteur, } i}$ une variable indicatrice qui est égale à 1 si le secteur de l'observation $i$ est le secteur testé et 0 sinon, $\mathrm{PD}_{j}$ est la probabilité de défaut du financement de projet $j, \alpha_{i}$ est une constante et $\beta_{i}$ est le coefficient de la deuxième régression. Les bêtas de la variable indicatrice de l'énergie des deux facteurs sont non significatifs à 10\%. Les bêtas individuels i sont significatifs à $10 \%$. Les deux SAR sont supérieurs à 0,3.

\begin{tabular}{|c|c|c|c|c|c|c|c|}
\hline FACTEUR & BETA ÉNERGIE & P VALUE & $\begin{array}{c}\text { BETA } \\
\text { PPP\&INFRA }\end{array}$ & P VALUE & SAR & BETA i & P VALUE \\
\hline Moyenne Marge EBIT & 0,0 & $84 \%$ & 0,1 & $0 \%$ & 0,42 & $-0,99$ & $0 \%$ \\
\hline Moyenne Marge EBITDA & 0,0 & $74 \%$ & 0,0 & $56 \%$ & 0,42 & $-0,66$ & $5 \%$ \\
\hline
\end{tabular}

À la lumière de ces résultats, les industries sont regroupées en trois sous-groupes, soient: Énergie, PPP et infra et Autres. Les transformations logistiques des facteurs sont faites par sous-groupe, sauf pour ce qui est de Marge EBIT et Marge EBITDA. Il est logique d'effectuer la transformation par sous-groupe de secteur car un certain niveau d'un ratio financier peut être bon dans une industrie et mauvais dans l'autre. Par exemple, un DSCR de 1,0 dans un PPP ne donne pas la même information qu'un DSCR de 1,0 dans un projet d'énergie. Dans l'échantillon, on remarque que les cotes de crédit des financements de projet dans le secteur des PPP et infra sont en moyenne supérieures aux cotes de crédit des projets dans d'autres secteurs. En effet, la cote moyenne du secteur PPP et infra est de BBB+ alors que celle du secteur Énergie et Autres sont respectivement $\mathrm{BB}+$ et $\mathrm{BBB}$-. Une variable indicatrice de secteur PPP et infra est donc incluse dans l'analyse multivariée.

\subsubsection{Traitements des variables macroéconomiques}

Pour les facteurs macroéconomiques, aucune transformation du type Moyenne du facteur sur une période maximale de trois ans n'est faite. Les facteurs macroéconomiques sont utilisés comme tels. Par contre, tout comme les facteurs financiers, une transformation logistique est appliquée à chacun des facteurs macroéconomiques.

\subsubsection{Traitements des données qualitatives}

Les scores des facteurs qualitatifs dans le gabarit sont sur une échelle de 1 à 3 où 1 est le pire score et 3 le meilleur score. Afin que les facteurs 
quantitatifs et le SCORE $E_{\text {Quant }}$ soient sur une échelle semblable, soit [0,1], les scores des facteurs qualitatifs sont ramenés sur une échelle $[0,1]$. Les scores de chacun des trois points de l'échelle qualitative sont attribués comme suit:

1) L'échantillon de développement qualitatif est divisé en trois tiers égaux de façon à ce que le premier tiers contienne les valeurs avec un centile plus petit ou égal à $33,33 \%$, le deuxième tiers les valeurs avec un centile plus petit ou égal à $66,66 \%$ mais plus grand que $33.33 \%$ et le troisième tiers les valeurs avec un centile plus grand que $66,66 \%$.

2) Ensuite, pour chacun des tiers, la valeur moyenne du score quantitatif est calculée. Ces valeurs sont les suivantes:

- premier tiers: 0,42;

- deuxième tiers : 0,59;

- troisième tiers : 0,82 .

Finalement, la valeur du score quantitatif moyen du premier tiers est attribuée au premier échelon de l'échelle qualitative, la valeur du score quantitatif moyen du deuxième tiers est attribuée au deuxième échelon de l'échelle qualitative et la valeur du score quantitatif moyen du troisième tiers est attribuée au troisième échelon de l'échelle qualitative.

\subsection{Analyse univariée des facteurs}

Maintenant que tous les facteurs sont correctement transformés, il est possible d'en faire l'analyse univariée. Dans cette section, le pouvoir explicatif de chacun des facteurs sur une base individuelle est analysé. Puis, la méthodologie et les résultats de l'analyse sont présentés.

\subsubsection{Critères de sélection des facteurs en analyse univariée}

Une fois les facteurs transformés, il faut établir des critères afin de sélectionner les facteurs qui ont le pouvoir explicatif le plus élevé. Un facteur est jugé avoir un pouvoir explicatif assez élevé pour passer à l'analyse multivariée lorsqu'il respecte les deux règles suivantes:

1) Le facteur a un $S A R>0,2$.

2) Le $\beta$ de la régression $\log (\mathrm{PD})=\alpha+\beta \mathrm{X}+\epsilon$ est significatif à $10 \%$ et de signe cohérent avec le signe attendu. 
Dans la régression du point $2, \alpha_{\mathrm{i}}$ est la constante de la régression du facteur $i, \beta_{i}$ est le bêta de la régression du facteur $i$ et $\mathrm{X}_{i}$ le facteur $i$.

Les deux conditions précédentes sont motivées comme suit:

- Comme il est démontré dans la section 4.4.3, un SAR inférieur à 0,2 est considéré comme un échec au niveau du pouvoir discriminant.

- La significativité du $\beta$ du facteur donne un bon indice sur le pouvoir explicatif de ce dernier.

\subsubsection{Résultats de l'analyse univariée des facteurs quantitatifs}

On retrouve dans le tableau 17 la liste des facteurs quantitatifs qui sont retenus par l'analyse univariée. Les résultats pour la liste complète des facteurs se trouvent à l'Annexe VI. Afin de conserver un aspect macroéconomique dans le modèle, le meilleur facteur des quatre facteurs macroéconomiques est conservé: il s'agit du taux directeur.

\section{TABLEAU 17 Facteurs quantitatifs retenus suite à l'analyse univariée de l'échantillon de développement}

Le SAR, le Beta de la régression et le $P$-Value du Beta de la régression sont présentés pour chaque facteur quantitatif retenu. Les $S A R$ sont $>0,2$ et les Betas sont tous significatif à $10 \%$. Les bêtas sont obtenus en faisant la régression $\log (\mathrm{PD})=\alpha_{i}+\beta_{i} X_{i}+\epsilon$ où $\alpha_{i}$ est la constante de la régression du facteur $i, \beta_{i}$ est le bêta de la régression du facteur $i$ et $X_{i}$ le facteur $i$.

\begin{tabular}{|c|c|c|c|c|}
\hline FACTEUR & $\begin{array}{l}\text { NOMBRE } \\
\text { D'OBSERVATION }\end{array}$ & SAR & BETA & P VALUE \\
\hline Moyenne du ratio de couverture du service de la dette & 481 & 0,41 & $-1,34$ & $0,00 \%$ \\
\hline Moyenne du ratio de dette sur EBITDA & 486 & 0,40 & $-0,96$ & $0,04 \%$ \\
\hline Moyenne du ratio de la dette par rapport aux capitaux propre & 486 & 0,28 & $-0,55$ & $3,29 \%$ \\
\hline Moyenne de la marge EBIT & 486 & 0,42 & $-0,64$ & $0,93 \%$ \\
\hline $\begin{array}{l}\text { Moyenne du logarithme du ratio de couverture du service } \\
\text { de la dette }\end{array}$ & 480 & 0,46 & $-1,22$ & $0,00 \%$ \\
\hline Moyenne du logarithme du ratio de couverture des intérêts & 473 & 0,41 & $-0,85$ & $0,06 \%$ \\
\hline Maximum du ratio de couverture du service de la dette & 481 & 0,41 & $-1,34$ & $0,00 \%$ \\
\hline Maximum du ratio des flux de trésorerie sur la dette & 480 & 0,34 & $-0,50$ & $8,75 \%$ \\
\hline Minimum du ratio de couverture du service de la dette & 481 & 0,39 & $-1,13$ & $0,00 \%$ \\
\hline $\begin{array}{l}\text { Moyenne du log-rendement au carré du ratio de couverture } \\
\text { de la dette }\end{array}$ & 469 & 0,12 & $-0,65$ & $2,64 \%$ \\
\hline Moyenne du log-rendement au carré de la marge EBIT & 442 & 0,43 & $-1,84$ & $0,00 \%$ \\
\hline Taux préférentiel & 461 & 0,17 & $-3,20$ & $0,00 \%$ \\
\hline
\end{tabular}


Afin de tester si l'inclusion d'une variable indicatrice du secteur PPP et infra serait bénéfique pour le modèle, nous faisons le même travail que précédemment mais en ajoutant cette dite variable. L'inclusion d'une variable indicatrice pour le secteur de l'énergie et l'inclusion des deux variables indicatrices sont également testées. La significativité des Betas ne change pas, mais les SAR sont supérieurs avec seulement la variable de PPP et l'infra. Voici donc les résultats. La régression prend la forme suivante: $\log (\mathrm{PD})=\alpha_{i}+\beta_{i} \mathrm{X}_{i}+\beta_{\mathrm{PPP} \& \text { Infra }} \mathrm{I}_{\mathrm{PPP} \& \text { Infra }}+\epsilon$ où $\alpha_{i}$ est la constante de la régression du facteur $i, \beta_{i}$ est le bêta de la régression du facteur $i, \mathrm{X}_{i}$ le facteur $i, \mathrm{I}_{\mathrm{PpP} \& \text { Infra }}$ une variable indicatrice du secteur PPP et infra et $\beta_{\text {PPP\&Infra }}$ le bêta de la variable indicatrice du secteur PPP et infra.

\section{TABLEAU 18 Facteurs quantitatifs retenus avec variable indicatrice du secteur PPP et infra de l'échantillon de développement}

Le SAR multivarié avec variable indicatrice du secteur PPP et infra, le Beta de la régression et le $P$-Value du Beta de la régression avec variable indicatrice du secteur PPP et infra sont présentés pour chaque facteur quantitatif retenu. Les Bêtas sont obtenus avec la régression: $\log (P D)=\alpha_{i}+\beta_{i} X_{i}+\beta_{\text {PpP\&lnfra }}$ lppp\&nnfra $_{\text {a }}+\epsilon$ où $\alpha_{i}$ est la constante de la régression du facteur $i, \beta_{i}$ est le bêta de la régression du facteur $i, X_{i}$ le facteur $i$, $I_{\text {PPP\&infra }}$ une variable indicatrice du secteur PPP et infra et $\beta_{\text {PPP\&lnfra }}$ le bêta de la variable indicatrice du secteur PPP et infra. Les SAR sont > 0,2 et les Betas sont tous significatifs à $10 \%$ sauf le facteur Moyenne de la marge EBIT.

\begin{tabular}{|c|c|c|c|c|}
\hline FACTEUR & $\begin{array}{l}\text { NOMBRE } \\
\text { D'OBSERVATION }\end{array}$ & $\begin{array}{l}\text { SAR } \\
\text { MULTI }\end{array}$ & BETA & P VALUE \\
\hline Moyenne du ratio de couverture du service de la dette & 481 & 0,69 & $-1,55$ & $0,00 \%$ \\
\hline Moyenne du ratio de dette sur EBITDA & 486 & 0,67 & $-1,12$ & $0,00 \%$ \\
\hline Moyenne du ratio de la dette par rapport aux capitaux propre & 486 & 0,60 & $-0,62$ & $0,60 \%$ \\
\hline Moyenne de la marge EBIT & 486 & 0,64 & $-0,34$ & $11,63 \%$ \\
\hline $\begin{array}{l}\text { Moyenne du logarithme du ratio de couverture du service } \\
\text { de la dette }\end{array}$ & 480 & 0,71 & $-1,42$ & $0,00 \%$ \\
\hline Moyenne du logarithme du ratio de couverture des intérêts & 473 & 0,67 & $-0,96$ & $0,00 \%$ \\
\hline Maximum du ratio de couverture du service de la dette & 481 & 0,70 & $-1,54$ & $0,00 \%$ \\
\hline Maximum du ratio des flux de trésorerie sur la dette & 480 & 0,66 & $-0,68$ & $0,86 \%$ \\
\hline Minimum du ratio de couverture du service de la dette & 481 & 0,67 & $-1,33$ & $0,00 \%$ \\
\hline $\begin{array}{l}\text { Moyenne du log-rendement au carré du ratio de couverture } \\
\text { de la dette }\end{array}$ & 469 & 0,55 & $-0,81$ & $0,15 \%$ \\
\hline Moyenne du log-rendement au carré de la marge EBIT & 442 & 0,65 & $-1,65$ & $0,00 \%$ \\
\hline Taux préférentiel & 461 & 0,69 & $-1,55$ & $0,00 \%$ \\
\hline
\end{tabular}


On remarque une nette augmentation du SAR du modèle. Il semble à première vue que l'ajout de la variable indicatrice augmente le pouvoir explicatif du modèle. De plus, tous les facteurs sélectionnés avant sont toujours significatifs à l'exception de la moyenne de la marge EBIT ${ }^{3}$.

\subsubsection{Résultats de l'analyse univariée des facteurs qualitatifs}

Malgré la petite taille de l'échantillon des facteurs qualitatifs, l'analyse univariée est tout de même faite et présentée dans cette section. Le tableau ci-dessous présente les résultats de cette analyse.

\section{TABLEAU 19 Résultats de l'analyse univariée des facteurs qualitatifs fait avec l'échantillon de développement du modèle qualitatif et quantitatif}

Seulement une année par contrepartie est considérée dans cette analyse. Seulement le facteur risque souverain et politique a un bêta significatif à $10 \%$. II est également le seul facteur à avoir un SAR plus grand que 0,30 et un $R^{2}$ plus grand que 0,10 .

\begin{tabular}{|c|c|c|c|c|c|}
\hline FACTEUR & $\begin{array}{c}\text { NOMBRE } \\
\text { D'OBSERVATIONS }\end{array}$ & SAR & BETA & P VALUE & $\mathbf{R}^{2}$ \\
\hline Risque de commanditaire & 29 & 0,10 & $-0,08$ & $61 \%$ & $1 \%$ \\
\hline Risque de construction & 29 & 0,02 & $-0,07$ & $63 \%$ & $1 \%$ \\
\hline Risque d'opération & 29 & 0,23 & $-0,10$ & $55 \%$ & $1 \%$ \\
\hline Risque de revenu & 29 & 0,11 & 0,02 & $92 \%$ & $0 \%$ \\
\hline Risque souverain et politique & 29 & 1,00 & $-1,25$ & $2 \%$ & $19 \%$ \\
\hline Risque d'industrie et de non-compétitivité & 29 & 0,13 & 0,09 & $82 \%$ & $0 \%$ \\
\hline Risque de liquidité & 29 & $-0,05$ & 0,13 & $72 \%$ & $0 \%$ \\
\hline Risque de caractéristiques de financement du projet & 29 & 0,19 & $-0,08$ & $81 \%$ & $0 \%$ \\
\hline
\end{tabular}

Suite à l'analyse des critères de sélection univariés des facteurs, le seul facteur qui semble avoir un pouvoir explicatif est le risque souverain et politique. Par contre, en regardant l'histogramme de distribution de ce facteur, on remarque que la majorité des observations ont un score de 3 pour ce dernier. À la lumière de l'analyse faite ci-haut, les facteurs qualitatifs ne semblent pas avoir un grand pouvoir explicatif. Par contre, les facteurs qualitatifs sont une partie importante de la notation de crédit. Ils se retrouvent dans la majorité des documentations de notation de crédit des grandes agences. Pour cette raison, les facteurs qualitatifs ne pouvaient pas être écartés du modèle. La section suivante présente l'analyse multivariée des facteurs. 


\subsection{Analyse multivariée des facteurs}

Maintenant l'analyse univariée terminée, on passe à l'analyse multivariée. À cette étape, des modèles comprenant plusieurs facteurs sont testés afin d'analyser la relation qu'ont les différents facteurs entre eux et d'identifier ceux qui ont le meilleur pouvoir explicatif. La méthode la plus simple est utilisée afin de tester les combinaisons de facteurs. Dans un premier temps, les facteurs qualitatifs sont écartés de l'analyse multivariée comme il a été démontré qu'une analyse purement statistique ne pouvait pas être faite sur ces derniers. L'approche utilisée consiste à tester toutes les combinaisons possibles de 2, 3, 4, 5, 6 et 7 facteurs quantitatifs inclus dans la liste finale de la section 6.4.2. La sélection des modèles pertinents se fait par après à l'aide de critères de sélections. L'un des critères de sélection est le SAR multivarié. Ce dernier est introduit dans la première partie de cette sous-section. Un autre critère de sélection des modèles est le Variance Inflation Factor (VIF) qui est introduit à la sous-section 6.5.2. Par la suite les autres critères discriminants et les résultats de l'analyse multivariée sont présentés.

\subsubsection{Introduction du SAR multivarié}

Tout comme dans l'analyse univariée, des régressions linéaires sont utilisées lors de l'analyse multivariée. Les régressions faites prennent la forme de l'équation 5 et ont la forme suivante:

$$
y=\alpha+\beta_{\text {Facteur } 1} x_{1}+\ldots+\beta_{\text {Facteur } n} x_{n}+\varepsilon .
$$

où $\alpha$ est la constante de la régression, $\mathrm{X}_{n}$ est la valeur du facteur étudié $n, \beta_{\text {Facteur } n}$ est le coefficients du facteur $n$ et $\varepsilon$ l'erreur d'estimation. Tel qu'expliqué à la section 4.3.1, les coefficients $\beta_{\text {Facteur } n}$ sont estimés en minimisant la somme des erreurs au carré. Dans le cas de l'approche utilisée, la variable dépendante de la régression multivariée est $\ln (\mathrm{PD})$. Ceci permet d'obtenir les PD estimées pour chaque observation $j$ pour chaque modèle $m$ à partir de l'équation 22 .

$$
\widehat{\mathrm{PD}}_{j, m}=e^{\hat{\alpha}_{m}+\hat{\beta}_{1, m} x_{1, j}+\ldots+\hat{\beta}_{n, m} x_{n, j}} .
$$

Comme $\widehat{\mathrm{PD}}_{j, m}$ est une estimation de $\mathrm{PD}_{j}$, il est logique de penser que si $\mathrm{PD}_{j}$ est élevée et que le modèle $m$ est le bon, alors $\widehat{\mathrm{PD}}_{j, m}$ sera également élevée. C'est donc dans cette optique que l'on utilise les $\widehat{\mathrm{PD}}_{j, m}$ comme facteurs afin de calculer le SAR multivarié. Le calcul du SAR multivarié se fait donc de la même façon que dans la section 4.4.3, mais on utilise l'estimation des PD au lieu des facteurs transformés sur $[0,1]$ dans le calcul. 


\subsubsection{Facteur d'inflation de la variance}

L'une des hypothèses structurelles de la régression linéaire est qu'il y a absence de colinéarité entre les variables explicatives. Il est donc important d'avoir une mesure afin de détecter ce phénomène. Le facteur d'inflation de la variance (VIF) est une mesure statistique qui permet de quantifier l'ampleur de la multicolinéarité dans une régression des moindres carrés ordinaires. On peut calculer le VIF du facteur $i$ dans une régression linéaire grâce à la formule

$$
\mathrm{VIF}_{i}=\frac{1}{1-\mathrm{R}_{i}^{2}}
$$

où $\mathbf{R}_{i}^{2}$ est le coefficient de détermination de l'équation

$$
\mathrm{X}_{i}=\beta_{0}+\beta_{1} \mathrm{X}_{1}+\beta_{2} \mathrm{X}_{2}+\ldots+\beta_{i-1} \mathrm{X}_{i-1}+\beta_{i+1} \mathrm{X}_{i+1}+\ldots+\beta_{n} \mathrm{X}_{n}+\varepsilon_{i} .
$$

Comme $\mathrm{R}_{i}^{2}$ représente la portion de la variance de $\mathrm{X}_{i}$ qui est expliquée par les autres facteurs, plus la dépendance linéaire entre les $\mathrm{X}_{i}$ et les autres variables est forte, plus la valeur $\mathrm{R}_{i}^{2}$ est grande et donc plus le VIF est élevé. Une valeur maximale de VIF doit donc être respectée afin de minimiser la présence de colinéarité entre les facteurs.

\subsubsection{Critères de sélection en analyse multivariée}

Afin de sélectionner les modèles les plus pertinents, plusieurs critères de sélections sont examinés. Les critères que doit respecter un modèle pour pouvoir être considéré comme bon sont les suivants:

1) Deux facteurs ayant un coefficient de corrélation de Spearman $>0,8$ sont fortement reliés entre eux et ne peuvent pas se retrouver dans le même modèle.

2) En statistique, il est généralement accepté que le seuil du VIF pour les problèmes de multicolinéarité soit de $2,5\left(\mathrm{R}_{i}^{2}<0,6\right)$ (Penn State Eberly College of Science). Tous les facteurs dans un modèle doivent donc avoir un $V I F$ inférieur à 2,5.

3) Les signes des $\beta$ dans la régression

$$
\log (\mathrm{PD})=\beta_{0}+\beta_{1} \mathrm{X}_{1}+\beta_{2} \mathrm{X}_{2}+\ldots+\beta_{7} \mathrm{X}_{7}+\varepsilon_{i}
$$

doivent être dans le sens attendu et significatif à $10 \%$.

4) Un SAR multivarié $>0,3$.

5) Un $R^{2}>0,10$. 
Falk (1992) recommande que la valeur du $\mathrm{R}^{2}$ soit plus grande ou égale à 0,10 pour être jugée adéquate. Tous les modèles qui répondent à ces critères sont étudiés afin de trouver ceux qui ont les meilleurs $\mathrm{R}^{2}$ et SAR multivariés. L'interprétation du coefficient de corrélation tirée d'Evans (1996) est présentée dans le tableau suivant.

\section{TABLEAU 20 Règle du pouce pour interpréter la hauteur du coefficient de corrélation tiré du texte d'Evans (1996)}

\begin{tabular}{|c|c|}
\hline GROSSEUR DE LA CORRÉLATION & INTERPRÉTATION \\
\hline 0,80 à $1,00(-0,80$ à $-1,00)$ & Corrélation positive (négative) très forte \\
\hline 0,60 à $0,79(-0,60$ à $-0,79)$ & Corrélation positive (négative) forte \\
\hline 0,40 à $0,59(-0,40$ à $-0,59)$ & Corrélation positive (négative) modérée \\
\hline 0,20 à $0,39(-0,20$ à $-0,39)$ & Corrélation positive (négative) faible \\
\hline 0,00 à $0,19(0,00$ à $-0,19)$ & Corrélation positive (négative) très faible à nulle \\
\hline
\end{tabular}

\subsubsection{Résultats de l'analyse multivariée des facteurs quantitatifs}

Avant d'examiner les modèles, nous débutons par l'analyse de la matrice de corrélation de Spearman afin de déterminer quels facteurs peuvent se retrouver ou non dans le même modèle (voir figure 22).

Suite à l'analyse de la matrice de corrélation, il est conclu que les combinaisons suivantes de facteurs ne peuvent pas se retrouver dans le même modèle.

- Moyenne du ratio de couverture du service de la dette \& Moyenne du logarithme du ratio de couverture de la dette

- Moyenne du ratio de couverture du service de la dette \& Maximum du ratio de couverture du service de la dette

- Moyenne du ratio de couverture du service de la dette \& Minimum du ratio de couverture du service de la dette

- Moyenne du logarithme du ratio de couverture de la dette \& Maximum du ratio de couverture du service de la dette

- Moyenne du logarithme du ratio de couverture de la dette \& Minimum du ratio de couverture du service de la dette 
FIGURE 22 Matrice de corrélation de Spearman de l'échantillon de développement des facteurs quantitatifs retenus lors de l'analyse univariée incluant la variable indicatrice du secteur PPP et infra

Les corrélations de Spearman supérieures à 0,8 sont en rouge. Les modèles ne peuvent donc pas contenir les combinaisons de facteurs suivantes: Moyenne du ratio de couverture du service de la dette \& Moyenne du logarithme du ratio de couverture de la dette; Moyenne du ratio de couverture du service de la dette \& Maximum du ratio de couverture du service de la dette; Moyenne du ratio de couverture du service de la dette \& Minimum du ratio de couverture du service de la dette ; Moyenne du logarithme du ratio de couverture de la dette \& Maximum du ratio de couverture du service de la dette et Moyenne du logarithme du ratio de couverture de la dette \& Minimum du ratio de couverture du service de la dette.

Moyenne du ratio de couverture du service de la dette Moyenne du ratio de dette sur EBITDA

Moyenne du ratio de la dette par rapport aux capitaux propre Moyenne de la marge EBIT

Moyenne du logarithme du ratio de couverture du service de la dette Moyenne du logarithme du ratio de couverture des intérêts Maximum du ratio de couverture du service de la dette Maximum du ratio des flux de trésorerie sur la dette Minimum du ratio de couverture du service de la dette Moyenne du log-rendement au carré du ratio de couverture de la dette Moyenne du log-rendement au carré de la marge EBIT Taux préférentiel

Variable Indicatrice du secteur PPP \& Infra

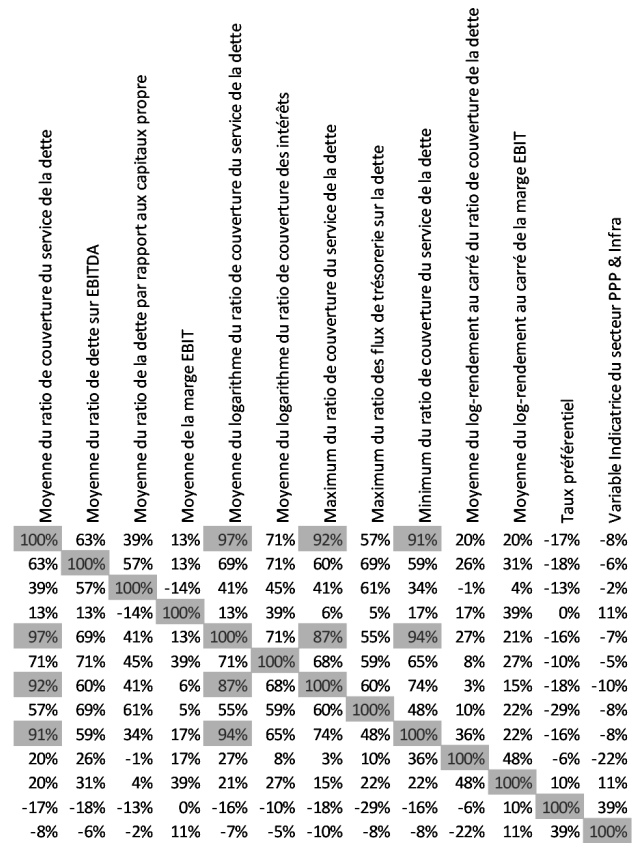

De plus, il est intéressant de constater que la variable indicatrice du secteur PPP et infra peut être dans le même modèle que tous les autres facteurs.

Suite à l'étude de tous les modèles de 2, 3, 4, 5, 6 et 7 facteurs, les quatre suivants sont sélectionnés. 


\section{TABLEAU 21 Modèles économétriques retenus $(Y=\log (P D))$ suite à l'analyse multivatriée de l'échantillon de développement}

Pour chaque modèle on retrouve la valeur du SAR multivarié du modèle et le $\mathrm{R}^{2}$ de la régression: $\log (P D)=\beta_{0}+\beta_{1} X_{1}+\beta_{2} X_{2}+\ldots+\beta_{7} X_{7}+\varepsilon_{i}$. Pour chaque facteur on retrouve son nom et sous son nom apparaît en ordre la valeur du Beta, la $P$-Value du Beta et le VIF du Beta. Tous les SAR multivariés sont supérieurs à 0,3. La relation entre chacun des facteurs et la probabilité de défaut est dans le sens attendu. Aucun VIF n'excède 2,5. Les $\mathrm{R}^{2}$ sont tous supérieurs à 0,10. Afin d'alléger le tableau, des noms abrégés sont utilisés dans le tableau ci-haut. Voici les noms abrégés et leurs définitions:

- PPP \& Infra = Variable indicatrice du secteur PPP et infra;

- DSCR = Moyenne du ratio de couverture du service de la dette;

- Log-rendement(DSCR ${ }^{2}=$ Moyenne du log-rendement au carré du ratio de couverture de la dette;

- Dette/EBITDA = Moyenne du ratio de dette sur EBITDA;

- Log-rendement(Marge EBIT) ${ }^{2}=$ Moyenne du log-rendement au carré de la marge EBIT;

- Marge EBIT = Moyenne de la marge EBIT;

- Dette/Capitaux propres = Moyenne du ratio de la dette par rapport aux capitaux propre.

\begin{tabular}{|c|c|c|c|c|c|c|c|c|}
\hline $\begin{array}{c}\mathrm{N}^{0} \mathrm{DE} \\
\text { MODÉLE }\end{array}$ & X1 & X2 & X3 & $X_{4}$ & X5 & X6 & SAR & $\mathrm{R}^{2}$ \\
\hline 1 & \begin{tabular}{|c} 
PPP \& INFRA \\
$-1,74813$ \\
$(<0,0001)$ \\
{$[1,05]$}
\end{tabular} & $\begin{array}{c}\text { DSCR } \\
-1,69015 \\
(<0,0001) \\
{[1,05]}\end{array}$ & $\begin{array}{c}\text { Log-rendement } \\
(\mathrm{DSCR})^{2} \\
-0,41499 \\
(0,09) \\
{[1,06]}\end{array}$ & $\begin{array}{c}\text { Taux Préférentiel } \\
-2,31814 x \\
(<0,0001) \\
{[1,04]}\end{array}$ & & & 0,70 & 0,36 \\
\hline 2 & \begin{tabular}{|c} 
PPP \& INFRA \\
$-1,57365$ \\
$(<0,0001)$ \\
{$[1,07]$}
\end{tabular} & $\begin{array}{c}\text { DSCR } \\
-1,50899 \\
(<0,0001) \\
{[1,06]}\end{array}$ & $\begin{array}{c}\text { Log-rendement } \\
\text { (Marge EBIT) }^{2} \\
-1,32438 \\
(<0,0001) \\
{[1,05]}\end{array}$ & $\begin{array}{l}\text { Taux Préférentiel } \\
-2,62510 \\
(<0,0001) \\
{[1,06]}\end{array}$ & & & 0,74 & 0,40 \\
\hline 3 & $\begin{array}{c}\text { PPP \& INFRA } \\
-1,57146 \\
(<0,0001) \\
{[1,07]}\end{array}$ & $\begin{array}{c}\text { DSCR } \\
-1,47469 \\
(<0,0001) \\
{[1,22]}\end{array}$ & $\begin{array}{c}\text { Dette/Capitaux } \\
\text { propres } \\
-0,08328 \\
(0,72) \\
{[1,17]}\end{array}$ & $\begin{array}{c}\begin{array}{c}\text { Log-rendement } \\
(\text { Marge EBIT) }\end{array} \\
-1,32588 \\
(<0,0001) \\
{[1,05]}\end{array}$ & $\begin{array}{c}\text { Taux Préférentiel } \\
-2,64231 \\
(<0,0001) \\
{[1,07]}\end{array}$ & & 0,73 & 0,40 \\
\hline 4 & $\begin{array}{c}\text { PPP \& INFRA } \\
-1,73659 \\
(<0,0001) \\
{[1,07]}\end{array}$ & $\begin{array}{c}\text { DSCR } \\
-1,59874 \\
(<0,0001) \\
{[1,94]}\end{array}$ & $\begin{array}{c}\text { Dette/EBITDA } \\
-0,12344 \\
(0,72) \\
{[2,10]}\end{array}$ & $\begin{array}{c}\text { Marge EBIT } \\
-0,07847 \\
(0,72) \\
{[1,07]}\end{array}$ & $\begin{array}{c}\text { Log-rendement } \\
\text { (DSCR) }^{2} \\
-0,38284 \\
(0,13) \\
{[1,12]}\end{array}$ & $\begin{array}{c}\text { Taux Préférentiel } \\
-2,35413 \\
(<0,0001) \\
{[1,07]}\end{array}$ & 0,71 & 0,36 \\
\hline
\end{tabular}

( ) : p-value, [ ] : VIF

Afin de prendre une décision finale quant au modèle, les performances hors échantillon de développement sont mesurées. La méthode et les résultats de ces mesures de performance des modèles sont présentés à la section 6.7 .

\subsubsection{Résultats de l'analyse multivariée des facteurs qualitatifs}

Comme les poids des facteurs qualitatifs ne pouvaient pas être déterminés avec une simple régression, une méthode alternative doit être utilisée pour faire l'analyse multivariée. Pour ce faire, une équipe 
d'expert a déterminé des poids pour chacun des facteurs qualitatifs et le poids global à attribuer aux facteurs quantitatifs. Le tableau suivant présente les poids sélectionnés.

\section{TABLEAU 22 Poids des facteurs pendant et après la période de construction déterminé par l'équipe d'experts}

Le risque de construction est seulement présent lorsque le projet est en construction, son poids est de zéro autrement. Les facteurs de risque de liquidité et risque de caractéristiques de financement du projet ont un poids de zéro. Tout comme dans Deluz (2010), ils sont utilisés pour faire un ajustement de cran à la note finale du modèle.

\begin{tabular}{|c|c|c|}
\hline FACTEUR & $\begin{array}{c}\text { POIDS SI NON } \\
\text { EN CONSTRUCTION }\end{array}$ & $\begin{array}{c}\text { POIDS SI } \\
\text { EN CONSTRUCTION }\end{array}$ \\
\hline $\mathrm{SCORE}_{\text {Quant }}$ & $60,00 \%$ & $60,00 \%$ \\
\hline Risque de commanditaire & $10,00 \%$ & $10,00 \%$ \\
\hline Risque d'opération & $7,50 \%$ & $2,50 \%$ \\
\hline Risque de revenu & $10,00 \%$ & $7,50 \%$ \\
\hline Risque d'industrie et de non-compétitivité & $5,00 \%$ & $2,50 \%$ \\
\hline Risque souverain et politique & $7,50 \%$ & $7,50 \%$ \\
\hline Risque de construction & $0,00 \%$ & $10,00 \%$ \\
\hline Risque de liquidité & $0,00 \%$ & $0,00 \%$ \\
\hline Risque de caractéristiques de financement du projet & $0,00 \%$ & $0,00 \%$ \\
\hline
\end{tabular}

Dans le tableau ci-haut, on remarque que les facteurs de risque de liquidité et de risque de caractéristiques de financement du projet ont un poids de $0,00 \%$. La raison de cela est que dans Deluz (2010), ces deux facteurs n'ont pas de poids attribué. Ils sont plutôt utilisés pour faire un ajustement de crans de la cote finale du modèle. La même méthodologie est appliquée à notre modèle. Les scores de ces facteurs peuvent uniquement diminuer la cote de crédit. Si le score de 1 est attribué à un de ces facteurs, la cote est diminuée d'un cran (notch). Si le score de 1 est attribué aux deux facteurs, la cote est diminuée de deux crans (notchs).

\subsection{Test du modèle}

Suite à l'analyse multivariée, quatre modèles sont retenus. Il faut maintenant en choisir un dans le lot. Afin de pouvoir comparer ces modèles, les quatre statistiques suivantes ont été comparées: 
1) Le SAR multivarié à l'intérieur de l'échantillon

2) Le SAR multivarié à l'extérieur de l'échantillon

3) L'espérance absolue du delta-cran $\mathrm{E}\left[\left|\Delta_{\text {Notch }}\right|\right]$ dans l'échantillon

4) L'espérance absolue du delta-cran $\mathrm{E}\left[\left|\Delta_{\text {Notch }}\right|\right]$ à l'extérieur de l'échantillon

Le delta-cran est la différence entre la cote de crédit réelle et la cote de crédit obtenue avec le modèle. Par exemple, si la cote de crédit réelle est AAA et la cote de crédit obtenue avec le modèle est $\mathrm{A}$, alors le delta-cran est de 2 . En plus des quatre statistiques présentées, les graphiques de dispersion des delta-cran $\left(\Delta_{\text {Notch }}\right)$ à l'intérieur et à l'extérieur de l'échantillon sont étudiés.

En premier lieu, la méthode de transformation des probabilités de défaut en cote de crédit est présentée. Ensuite, l'espérance absolue du delta-cran comme mesure de performance d'un modèle de notation de crédit est introduite. Par la suite, les résultats des quatre statistiques présentées ci-haut et les graphiques des delta-cran sont présentés. Subséquemment, les motivations derrière le choix du modèle quantitatif final sont exposées. Finalement, les facteurs qualitatifs sont ajoutés au modèle final dans les deux dernières sous-sections.

\subsubsection{Transformation des PD en échelle alpha numérique}

Une des façons d'analyser la performance d'un modèle, est de comparer les cotes de crédit réelles avec celles générées par le modèle. Pour ce faire, il faut transformer les probabilités de défaut issues du modèle en échelle alphanumérique. La table de transition présentée à la section 6.1 est utilisée pour faire ce travail. La même méthode que dans Bauer (2013) est utilisée pour calculer les probabilités limites de chaque cote de risque. La formule utilisée est la suivante:

$$
\mathrm{PD}_{\text {Limite, } \text { Cote }=x}=\sqrt{\mathrm{PD}_{x} * \mathrm{PD}_{x-1}}
$$

où $\mathrm{PD}_{\text {Limite, Cote }=x}$ est la probabilité de défaut limite supérieure de la cote de crédit $x$ lors de la conversion des PD du modèle en cotes de crédit, $\mathrm{PD}_{x}$ est la probabilité de défaut associée à la cote de crédit $x$ et $\mathrm{PD}_{x-1}$ est la probabilité de défaut associée à la cote de crédit précédent $x$. Ce qui donne les probabilités limites par cote de risque suivantes: 
TABLEAU 23 Table des probabilités de défaut et des probabilités de défaut limites par cote de risque calculées avec la méthode tirée de Bauer (2013)

Les probabilités de défaut limites sont calculées avec $\mathrm{PD}_{\text {Limite, Cote }=x}=\sqrt{\mathrm{PD}_{x} * \mathrm{PD}_{x-1}}$ où $\mathrm{PD}_{\text {Limite, cote }=x}$ est la probabilité de défaut limite supérieure de la cote de crédit $x$ lors de la conversion des PD du modèle en cotes de crédit, $\mathrm{PD}_{x}$ est la probabilité de défaut associée à la cote de crédit $x$ et $\mathrm{PD}_{x-1}$ est la probabilité de défaut associée à la cote de crédit précédent $x$.

\begin{tabular}{|c|c|c|}
\hline COTES DE RISQUE* & PD & $\mathrm{PD}_{\mathrm{LIM}}$ \\
\hline AAA & $0,00010 \%$ & $0 \%<\mathrm{PD}_{\text {Modèle }} \leq 0,00018 \%$ \\
\hline AAH & $0,00033 \%$ & $0,00018 \%<\mathrm{PD}_{\text {Modele }} \leq 0,00061 \%$ \\
\hline AA & $0,00110 \%$ & $0,00061 \%<\mathrm{PD}_{\text {Modèle }} \leq 0,00201 \%$ \\
\hline AAL & $0,00367 \%$ & $0,00201 \%<\mathrm{PD}_{\text {Modele }} \leq 0,00669 \%$ \\
\hline AH & $0,01220 \%$ & $0,00669 \%<\mathrm{PD}_{\text {Modele }} \leq 0,02225 \%$ \\
\hline$A$ & $0,04056 \%$ & $0,02225 \%<\mathrm{PD}_{\text {Modele }} \leq 0,05333 \%$ \\
\hline $\mathrm{AL}$ & $0,07012 \%$ & $0,05333 \%<\mathrm{PD}_{\text {Modèle }} \leq 0,11518 \%$ \\
\hline BBBH & $0,18919 \%$ & $0,11518 \%<\mathrm{PD}_{\text {Modele }} \leq 0,20093 \%$ \\
\hline BBB & $0,21341 \%$ & $0,20093 \%<\mathrm{PD}_{\text {Modèle }} \leq 0,24156 \%$ \\
\hline BBBL & $0,27343 \%$ & $0,24156 \%<\mathrm{PD}_{\text {Modèle }} \leq 0,33147 \%$ \\
\hline $\mathrm{BBH}$ & $0,40182 \%$ & $0,33147 \%<\mathrm{PD}_{\text {Modele }} \leq 0,52497 \%$ \\
\hline BB & $0,68586 \%$ & $0,52497 \%<\mathrm{PD}_{\text {Modele }} \leq 0,82040 \%$ \\
\hline BBL & $0,98132 \%$ & $0,82040 \%<\mathrm{PD}_{\text {Modele }} \leq 1,12901 \%$ \\
\hline $\mathrm{BH}$ & $1,29893 \%$ & $1,12901 \%<\mathrm{PD}_{\text {Modèle }} \leq 1,91954 \%$ \\
\hline B & $2,83666 \%$ & $1,91954 \%<\mathrm{PD}_{\text {Modele }} \leq 3,33510 \%$ \\
\hline $\mathrm{BL}$ & $3,92112 \%$ & $3,33510 \%<\mathrm{PD}_{\text {Modele }} \leq 5,06136 \%$ \\
\hline $\mathrm{CCCH}$ & $6,53316 \%$ & $5,06136 \%<\mathrm{PD}_{\text {Modèle }} \leq 10,33394 \%$ \\
\hline $\mathrm{CCC}$ & $16,34589 \%$ & $10,33394 \%<\mathrm{PD}_{\text {Modèle }} \leq 22,19457 \%$ \\
\hline CCCL & $30,13595 \%$ & $22,19457 \%<\mathrm{PD}_{\text {Modèle }} \leq 37,05812 \%$ \\
\hline CC-C & $45,57029 \%$ & $37,05812 \%<\mathrm{PD}_{\text {Modèle }} \leq 100 \%$ \\
\hline
\end{tabular}

* Échelle DBRS utilisé car c'est l'échelle qui était déjà utilisée à l'interne

La cote de crédit du modèle peut donc être obtenue à partir de la PD du modèle et de la table ci-haut. Par la suite, cette cote peut être comparée avec la cote moyenne du projet calculée avec la méthodologie présentée à la section 6.3.1. Les résultats de ces comparaisons sont présentés dans la section suivante. 
L'espérance absolue du delta-cran est une mesure qui est utilisée afin de comparer des modèles entre eux dans Metz (2006). Pour calculer l'espérance absolue du delta-cran d'un échantillon, il faut commencer par ramener les cotes de crédit alpha numériques [AAA, CC-C] sur une échelle numérique [1-20] afin de pouvoir soustraire les cotes réelles et les cotes du modèle. Une fois les cotes de crédit sur une échelle numérique, il est possible, grâce à l'équation 26 de calculer pour chacune des observations $i$, la différence entre la cote de crédit numérique réelle équivalente à DBRS et la cote numérique du modèle.

La formule est:

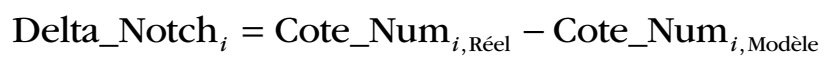

où Cote_Num $i$,Réel est la cote numérique réelle et Cote_Num $i$,Modèle celle obtenue grâce au modèle. Les histogrammes des delta-cran $\left(\Delta_{\text {Notch }}\right)$ à l'intérieur et à l'extérieur de l'échantillon peuvent ensuite être tracés. Plus les delta-cran du modèle sont centrés près de 0 , meilleur est le modèle pour répliquer les cotes de crédit externes, car moins les cotes du modèle dévient des cotes réelles des agences de notation. Avec l'équation 27, il est par la suite possible de calculer l'espérance absolue du delta-cran $\left(\Delta_{\text {Notch }}\right)$ à l'intérieur et à l'extérieur de l'échantillon.

$$
\mathrm{E}\left[\left|\Delta_{\text {Notch }}\right|\right]=\sum_{i=1}^{\text {nb obs }} \frac{\mid \text { Delta_Notch }}{i} \mid
$$

Encore une fois, plus l'espérance absolue du delta-cran $\left(\Delta_{\text {Notch }}\right)$ à l'intérieur de l'échantillon et l'espérance absolue du delta-cran à l'extérieur de l'échantillon se rapproche de 0 , meilleur est le modèle.

\subsubsection{Résultats quantitatifs}

Les résultats de l'analyse de performance de chaque modèle sont présentés dans les figures 23 à 30 . 
FIGURE 23 Histogrammes des delta-cran à l'intérieur de l'échantillon de développement du modèle 1

L'espérance absolue du delta-cran à l'intérieur de l'échantillon est de 2,193 et le SAR à l'intérieur de l'échantillon est de 0,698.

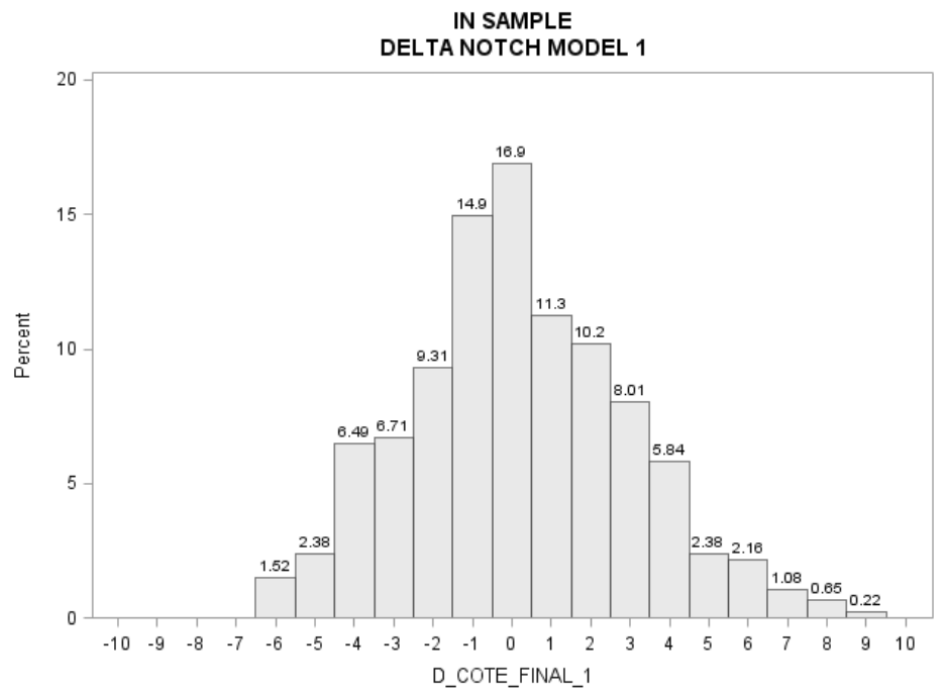

\section{FIGURE 24 Histogrammes des delta-cran à l'extérieur de l'échantillon de développement du modèle 1}

L'espérance absolue du delta-cran à l'extérieur de l'échantillon est de 2,431 et le SAR à l'extérieur de l'échantillon est de 0,592.

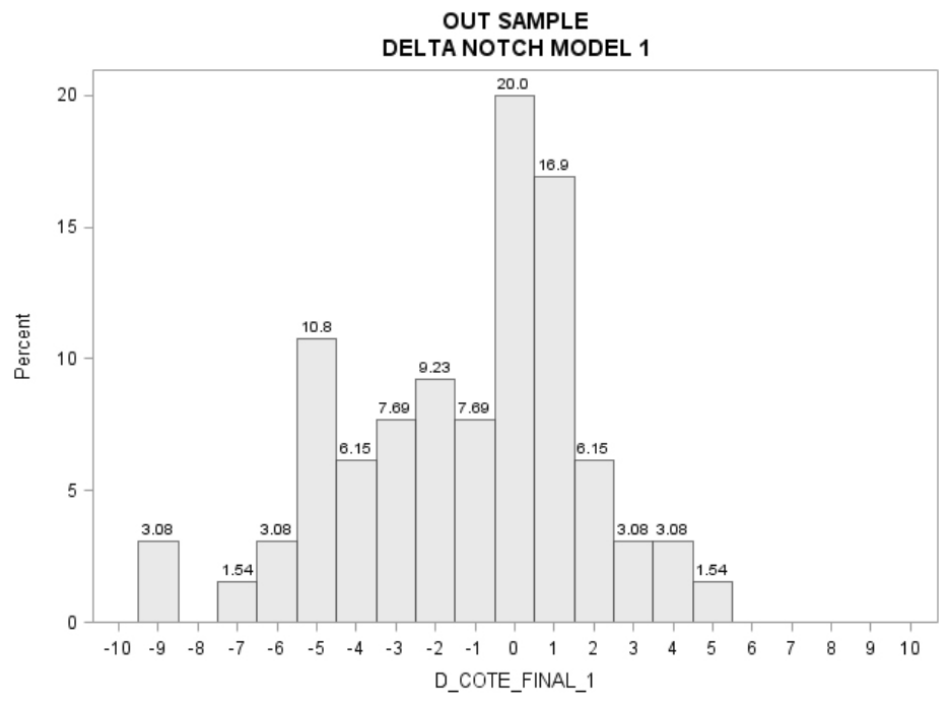


FIGURE 25 Histogrammes des delta-cran à l'intérieur de l'échantillon de développement du modèle 2

L'espérance absolue du delta-cran à l'intérieur de l'échantillon est de 2,074 et le SAR à l'intérieur de l'échantillon est de 0,735.

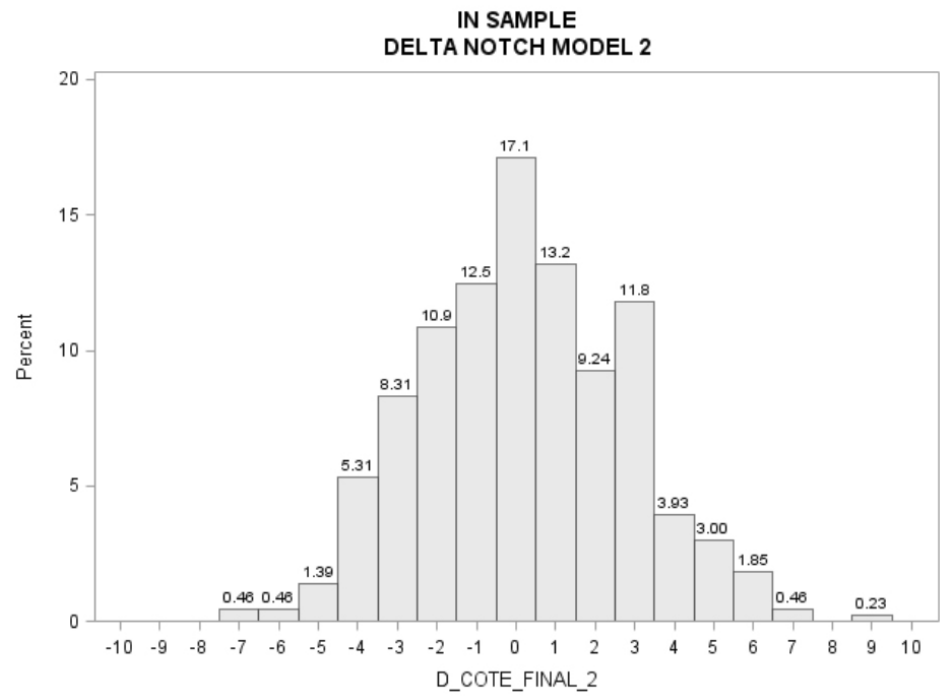

\section{FIGURE 26 Histogrammes des delta-cran à l'extérieur de l'échantillon de développement du modèle 2}

L'espérance absolue du delta-cran à l'extérieur de l'échantillon est de 2,651 et le SAR à l'extérieur de l'échantillon est de 0,381.

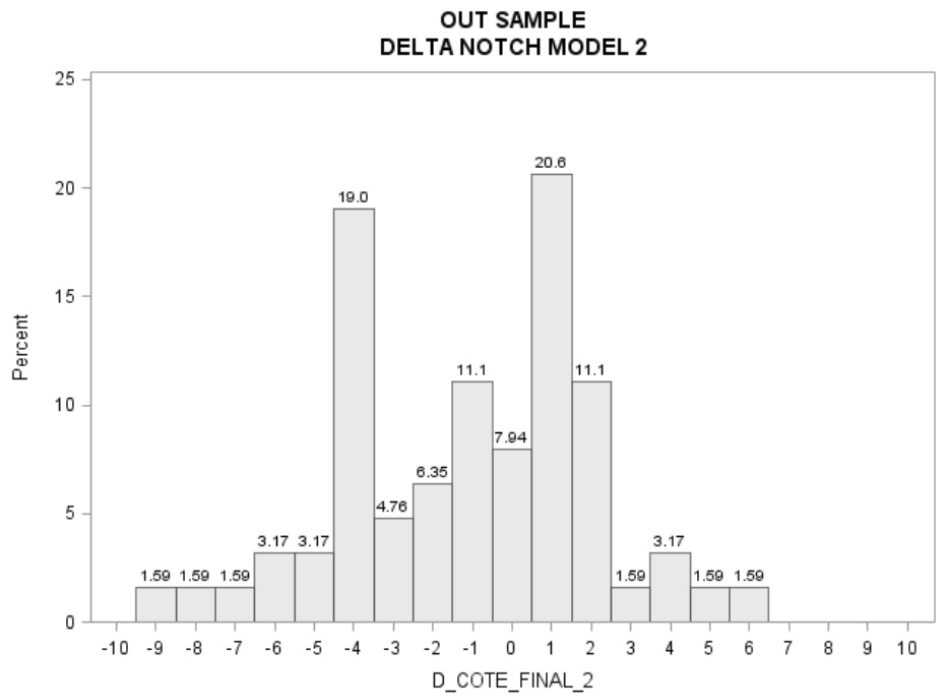


FIGURE 27 Histogrammes des delta-cran à l'intérieur de l'échantillon de développement du modèle 3

L'espérance absolue du delta-cran à l'intérieur de l'échantillon est de 2,060 et le SAR à l'intérieur de l'échantillon est de 0,732.

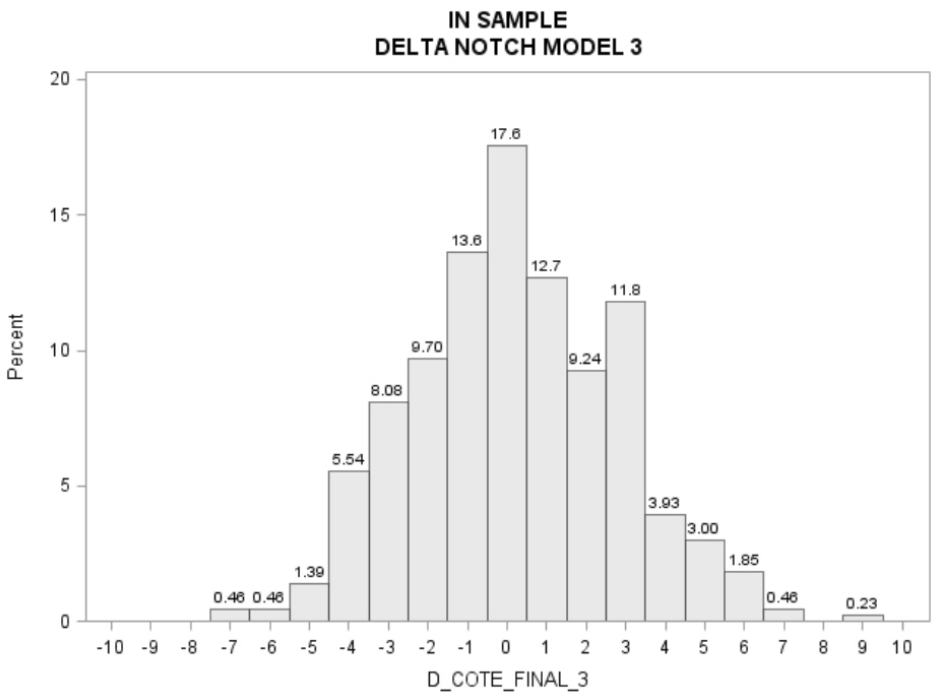

\section{FIGURE 28 Histogrammes des delta-cran à l'extérieur de l'échantillon de développement du modèle 3}

L'espérance absolue du delta-cran à l'extérieur de l'échantillon est de 2,635 et le SAR à l'extérieur de l'échantillon est de 0,388.

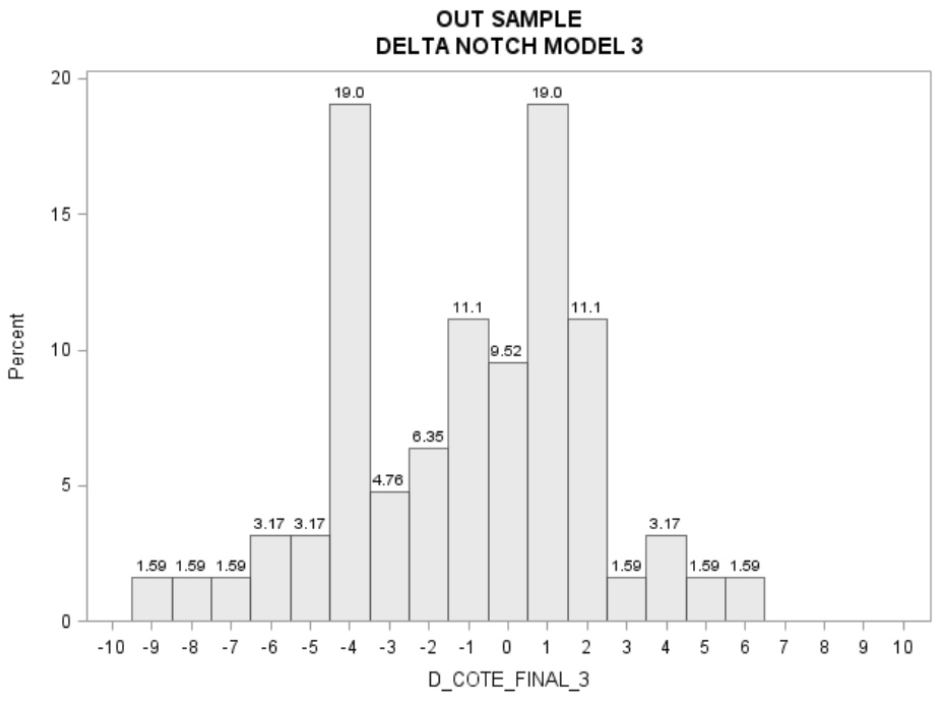


FIGURE 29 Histogrammes des delta-cran à l'intérieur de l'échantillon de développement du modèle 4

L'espérance absolue du delta-cran à l'intérieur de l'échantillon est de 2,184 et le SAR à l'intérieur de l'échantillon est de 0,712.

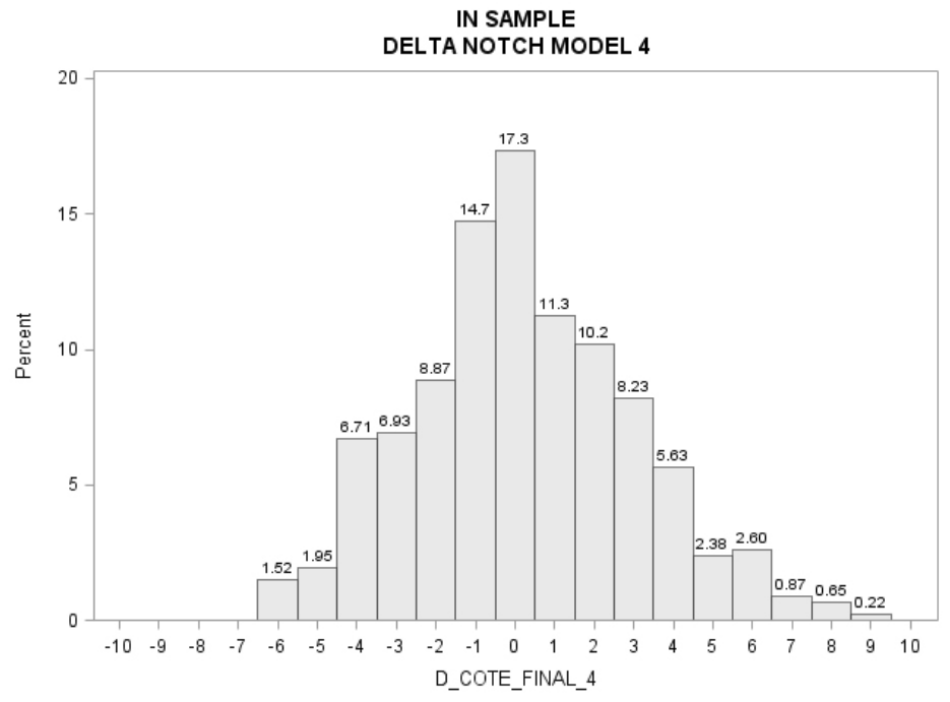

\section{FIGURE 30 Histogrammes des delta-cran à l'extérieur de l'échantillon de développement du modèle 4}

L'espérance absolue du delta-cran à l'extérieur de l'échantillon est de 2,462 et le SAR à l'extérieur de l'échantillon est de 0,590.

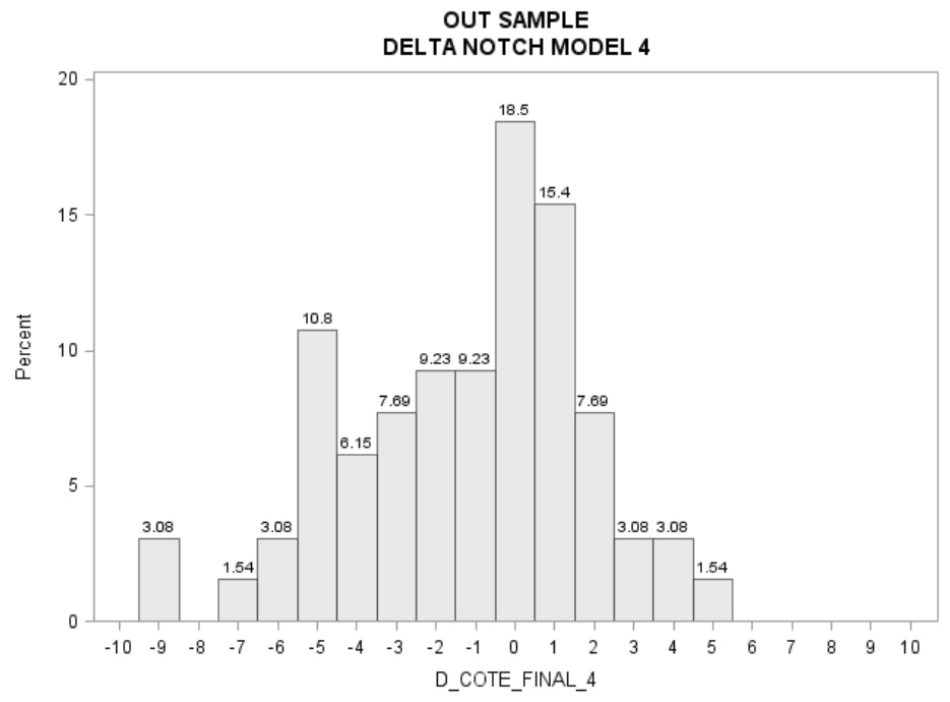


En comparant les modèles ci-haut entre eux, on remarque que les modèles 2 et 3 performent mieux à l'intérieur de l'échantillon que les modèles 1 et 4 . En effet, les modèles 2 et 3 ont respectivement des SAR à l'intérieur de l'échantillon de 0,735 et 0,732 comparativement aux modèles 1 et 4 qui eux ont respectivement des SAR à l'intérieur de l'échantillon de 0,698 et 0,712 . Lorsque l'on regarde les $\mathrm{E}\left[\left|\Delta_{\text {Notch }}\right|\right]$ à l'intérieur de l'échantillon, on observe la même chose. Les modèles 2 et 3 ont respectivement des $\mathrm{E}\left[\left|\Delta_{\text {Notch }}\right|\right]$ à l'intérieur de l'échantillon de 2,073 et 2,060 . Ils ont donc des $\mathrm{E}\left[\left|\Delta_{\text {Notch }}\right|\right]$ à l'intérieur de l'échantillon inférieur à ceux des modèles 1 et 4 qui sont respectivement 2,193 et 2,184 .

Afin de voir si les modèles performent bien en dehors de l'échantillon, les mêmes statistiques sont calculées pour les modèles 1, 2, 3 et 4 dans un échantillon autre que celui de développement. Cet échantillon est l'échantillon de validation. En analysant les résultats, on observe que, à l'inverse des résultats des statistiques dans l'échantillon, les modèles 1 et 4 performent mieux que les modèles 2 et 3 dans l'échantillon de validation. Le SAR dans l'échantillon de validation du modèle 1 est de 0,592, celui du modèle 2 est de 0,381 , celui du modèle 3 est de 0,388 et celui du modèle 4 est de 0,590 . Pour ce qui est de $\mathrm{E}\left[\left|\Delta_{\text {Notch }}\right|\right]$, la mesure est de 2,431 pour le modèle 1 , de 2,651 pour le modèle 2 , de 2,635 pour le modèle 3 et de 2,462 pour le modèle 4 . Comme il est important qu'un modèle performe bien à l'intérieur et à l'extérieur de l'échantillon, les modèles 1 et 4 sont préférés aux modèles 2 et 3 .

Comme le but de l'essai est de construire un modèle, il faut maintenant choisir entre le modèle 1 et le modèle 4 . La présence dans le modèle 4 des ratios Dette/EBITDA et Marge EBIT, malgré qu'ils soient non significatifs, amène de l'information qui n'est pas couverte par le $D S C R$ et la variation du DSCR. Plus précisément, le ratio Dette/EBITDA donne une approximation du nombre d'années que prendra la compagnie à rembourser ses dettes. Cela n'est pas capté par le $D S C R$ qui lui donne plutôt une indication que la compagnie est capable de payer ses dettes ou non dans l'immédiat. Pour ce qui est du ratio Marge EBIT, il aide à comprendre les coûts réels d'affaires de la gestion de la compagnie. Encore une fois un aspect que le $D S C R$ ne capte pas. Pour ces raisons, le modèle 4 est choisi comme modèle quantitatif final.

Une fois le modèle quantitatif final choisi, les $n$ coefficients de la régression sont transformés avec l'équation 28 afin que leur somme donne 1 . De cette façon, on peut voir le pourcentage du poids qu'a chaque facteur dans la régression. 


$$
\text { Poids }_{i}=\frac{\beta_{i}}{\sum_{j=1}^{n} \beta j}
$$

où $\beta_{i}$ est le bêta du facteur $i$ dans le modèle 4 et Poids $s_{i}$ est le poids en pourcentage dans le modèle 4 du facteur $i$.

Les poids en pourcentage des facteurs du modèle quantitatif final sont présentés dans le tableau suivant. On remarque que le facteur de secteur PPP\&INFRA et le facteur Taux Préférentiel représentent à eux seuls $65,20 \%$ du poids total des facteurs. Cela laisse croire que le secteur et les taux d'intérêt ont beaucoup d'importance sur la notation de crédit du financement de projets. Dans les 34,8\% restant du poids, $25,48 \%$ est au DSCR et 6,10\% au Log-rendement (DSCR) ${ }^{2}$. Cela porte à croire qu'un DSCR élevé et stable dans le temps est un indicateur d'une bonne qualité de crédit.

\section{TABLEAU 24 Poids en pourcentage des facteurs du modèle quantitatif final}

Le facteur de secteur PPP\&INFRA et le facteur Taux Préférentiel représentent à eux seuls 65,20\% du poids total des facteurs. Le DSCR et 6,10\% au Log-rendement(DSCR)2 , quant à eux représentent $31,58 \%$ du poids total.

\begin{tabular}{|c|c|c|c|c|c|}
\hline PPP \& INFRA & MARGE EBIT & DSCR & DETTE/EBITDA & $\begin{array}{l}\text { LOG-RENDEMENT } \\
(\text { DSCR) }\end{array}$ & $\begin{array}{c}\text { TAUX } \\
\text { PRÉFÉRENTIEL }\end{array}$ \\
\hline $27,68 \%$ & $1,97 \%$ & $25,48 \%$ & $1,25 \%$ & $6,10 \%$ & $37,52 \%$ \\
\hline
\end{tabular}

\subsubsection{Ajout des facteurs qualitatifs au modèle}

Maintenant que le modèle quantitatif a été choisi, il s'agit d'inclure les facteurs qualitatifs. Tous les facteurs de la régression 30 sont mis sur le même domaine afin de faciliter la comparaison des bêtas de la régression entre eux. Tous les facteurs seront donc sur le domaine $[0,1]$. Pour ce faire, le $\mathrm{SCORE}_{\mathrm{Quant}}$ calculé avec l'équation suivante,

$$
\mathrm{SCORE}_{\text {Quant }}=\sum_{i=1}^{n} \text { Poids }_{i} * \text { Facteur }_{i}
$$

où Poids $s_{i}$ est le poids en pourcentage du facteur $i$ calculé avec l'équation 28 et Facteur $_{i}$ est le facteurs quantitatif $i$ ayant subi une transformation logistique. Le domaine de $\mathrm{SCORE}_{\text {Quant }}$ est donc $[0,1]$. 
Le $\mathrm{SCORE}_{\text {Quant }}$ est utilisé comme un facteur dans le modèle qui inclut les facteurs quantitatifs et qualitatifs de la façon suivante:

$\log (\mathrm{PD})=\beta_{0}+\beta_{\text {Quant }} \mathrm{SCORE}_{\text {Quant }}+\beta_{1, \text { Qual }} \mathrm{X}_{1, \text { Qual }}+\ldots+\beta_{n, \text { Qual }} \mathrm{X}_{n, \text { Qual }}+\varepsilon$.

À partir de l'équation (30), on obtient le modèle final qui est présenté à la figure 31 .

\section{FIGURE 31 Modèle final de notation de financement de projet} avec poids des facteurs quantitatifs et qualitatifs et ce pour les projets en construction et non en construction

\begin{tabular}{|c|c|c|c|c|}
\hline FACTEURS & $\begin{array}{l}\text { POIDS DES } \\
\text { FACTEURS }\end{array}$ & SOUS-FACTEURS & $\begin{array}{l}\text { POIDS DES SOUS- } \\
\text { FACTEURS EN } \\
\text { CONSTRUCTION }\end{array}$ & $\begin{array}{c}\text { POIDS DES } \\
\text { SOUS-FACTEURS } \\
\text { NON EN } \\
\text { CONSTRUCTION }\end{array}$ \\
\hline \multirow[t]{6}{*}{ Quantitatif } & \multirow[t]{6}{*}{$60 \%$} & Variable indicatrice de projet PPP \& Infra & $16,61 \%$ & $16,61 \%$ \\
\hline & & Moyenne 3 ans maximum de la marge EBIT & $1,18 \%$ & $1,18 \%$ \\
\hline & & Moyenne 3 ans maximum du DSCR & $15,29 \%$ & $15,29 \%$ \\
\hline & & $\begin{array}{l}\text { Moyenne } 3 \text { ans maximum du ratio dette } \\
\text { portant intérêt / EBITDA }\end{array}$ & $0,75 \%$ & $0,75 \%$ \\
\hline & & $\begin{array}{l}\text { Moyenne maximum } 2 \text { ans des log } \\
\text { rendements au carré du DSCR }\end{array}$ & $3,66 \%$ & $3,66 \%$ \\
\hline & & Taux préférentiel du pays & $22,51 \%$ & $22,51 \%$ \\
\hline \multirow[t]{6}{*}{ Qualitatif } & \multirow[t]{6}{*}{$40 \%$} & Risque de commanditaire & $10,00 \%$ & $10,00 \%$ \\
\hline & & Risque d'opération & $2,50 \%$ & $7,50 \%$ \\
\hline & & Risque de revenu & $7,50 \%$ & $10,00 \%$ \\
\hline & & Risque d'industrie et de non-compétitivité & $2,50 \%$ & $5,00 \%$ \\
\hline & & Risque souverain et politique & $7,50 \%$ & $7,50 \%$ \\
\hline & & Risque de construction & $10,00 \%$ & $0,00 \%$ \\
\hline
\end{tabular}

Afin de simplifier l'interprétation et la comparaison des scores de crédit extraits du modèle, ces derniers sont ramenés sur une échelle finie $[0,1]$. Comme chacun des facteurs est sur un domaine $[0,1]$, on peut obtenir avec l'équation 31 et les poids du tableau précédent, le SCORE d'un projet. Ce dernier sera également sur le domaine $[0,1]$. Cette transformation facilitera l'ajustement final du SCORE aux probabilités de défaut. Par la suite, la probabilité de défaut sous-jacente pourra être transformée en cote alpha numérique.

$$
\text { SCORE }=\sum_{i=1}^{n+m} \text { Poids }_{i} * \text { Facteur }_{i}
$$


où SCORE est le score sur $[0,1]$ d'un projet, Poids ${ }_{i}$ est le poids du facteur $i$, Facteur $_{i}$ est le facteur $i$ transformé sur $[0,1]$ et $n$ le nombre de facteurs quantitatifs et $m$ le nombre de facteurs qualitatifs. La transformation de ce SCORE en cote alpha numérique est abordée dans la section suivante.

\subsubsection{Ajustement exponentiel}

Comme nous faisons l'hypothèse que la relation entre les cotes de crédit et les probabilités de défaut associées sont exponentielles, il est logique de faire la même chose avec notre SCORE. C'est-à-dire, de faire un ajustement exponentiel entre la probabilité de défaut associée au projet et son SCORE. De cette façon, le modèle donne une probabilité de défaut qui peut ensuite être transformée en cote de crédit. L'ajustement exponentiel fait dans le cadre de notre modèle est présenté dans cette section.

L'hypothèse faite plus tôt peut être traduite dans l'équation suivante:

$$
\mathrm{PD}_{i}=e^{\alpha_{1}+\beta_{1} * \text { Cote numérique }_{i}},
$$

où $\mathrm{PD}_{i}$ est la probabilité de défaut de la contrepartie $i, \alpha_{1}$ est une constante, $\beta_{1}$ est le paramètre de la fonction exponentielle et Cote numérique $_{i}$ la cote de crédit de la contrepartie $i$ ramenée sur une échelle numérique. Le même parallèle est fait entre $\mathrm{PD}_{i}$ et $\mathrm{SCORE}_{i}$ dans l'équation suivante:

$$
\mathrm{PD}_{i}=e^{\alpha_{2}+\beta_{2} * \mathrm{SCORE}_{i}},
$$

où $\mathrm{PD}_{i}$ est la probabilité de défaut de la contrepartie $i, \alpha_{2}$ est une constante, $\beta_{2}$ est le paramètre de la fonction exponentielle et $\operatorname{SCORE}_{i}$ est le score de la contrepartie $i$ obtenu avec l'équation 31 .

\section{RÉSULTATS}

Comme le modèle final comporte des facteurs dont le pouvoir explicatif n'a pas été statistiquement démontré, il faut trouver une façon d'infirmer ou de confirmer si le choix des facteurs du modèle est bon ou non. Pour ce faire, le modèle est comparé avec 60 modèles alternatifs. Les modèles sont les suivants:

- Modèles avec différents poids sur les facteurs quantitatifs et une répartition équipondérée sur les facteurs qualitatifs (cinq modèles). 
- Modèles avec différents poids sur les facteurs quantitatifs et la répartition faite par des experts sur les facteurs qualitatifs (quatre modèles).

- Modèles avec différents poids sur les facteurs quantitatifs et des répartitions aléatoires du poids sur les facteurs qualitatifs (50 modèles).

- Modèle avec tout le poids sur les facteurs quantitatifs (un modèle).

Par la suite, les statistiques suivantes des différents modèles sont comparées entre elles afin de déterminer quel modèle est le meilleur:

- le SAR;

- l'erreur quadratique moyenne (MSE) des cotes de crédit numériques;

- la corrélation de Spearman entre les cotes numériques estimées et les cotes numériques réelles.

Les valeurs de chacune des statistiques ci-haut sont ordonnées de la meilleure valeur à la pire. Pour chaque statistique, la meilleure valeur se voit attribuer le rang 1 , la deuxième meilleure valeur de rang 2 et ainsi de suite jusqu'à la dernière valeur qui se voit attribuer le dernier rang. Ensuite, le rang moyen de chacun des modèles est calculé de la façon suivante:

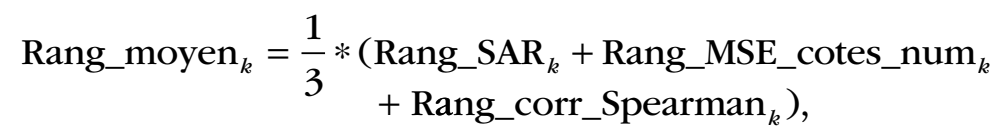

où Rang_moyen ${ }_{k}$ est le rang moyen des statistiques du modèle $k$, Rang_SAR $k$ est le rang du SAR du modèle $k$, Rang_MSE_cotes_num ${ }_{k}$ est le rang de l'erreur quadratique moyenne des cotes de crédit numériques du modèle $k$ et Rang_corr_Spearman ${ }_{k}$ est le rang de la corrélation de Spearman entre les cotes numériques estimées et les cotes numériques réelles du modèle $k$. Les rangs moyens calculés avec l'équation 34 sont ensuite ordonnés du plus petit au plus grand. Le modèle avec le rang moyen le plus faible est considéré comme étant le meilleur. Le tableau 25 présente les résultats de l'analyse. On remarque que le modèle expert arrive troisième sur 61 lorsque classé par rang moyen. Il a un SAR de 0,34, une erreur quadratique moyenne des cotes de crédit numériques de 1,38 et une corrélation de Spearman entre les cotes numériques estimées et les cotes numériques réelles de 0,31. Ce qui lui donne respectivement les rangs de 23, 19 et 4 pour chacune des mesures. Quant à lui, le modèle avec tout le poids sur les facteurs quantitatifs arrive $47^{\text {ème }}$ sur les 61 modèles, ce qui est un rang 


\section{TABLEAU 25 Résultats partiels de l'analyse comparative entre le modèle expert et des modèles alternatifs}

On remarque que le modèle expert est troisième sur 61 modèles. II a un SAR de 0,34, une erreur quadratique moyenne des cotes de crédit numériques de 1,38 et une corrélation de Spearman entre les cotes numériques estimées et les cotes numériques réelles de 0,31. Le modèle avec tout le poids sur les facteurs quantitatifs arrive 47 ème sur les 61 modèles avec un $S A R$ de 0,32, une erreur quadratique moyenne des cotes de crédit numériques de 1,71 et une corrélation de Spearman entre les cotes numériques estimées et les cotes numériques réelles de 0,25. Les valeurs de chacune des statistiques du tableau sont ordonnées de la meilleure valeur à la pire. Pour chaque statistique, la meilleure valeur se voit attribuer le rang 1, la deuxième meilleure valeur de rang 2 et ainsi de suite jusqu'à la dernière valeur qui se voit attribuer le dernier rang. Ensuite, le rang moyen de chacun des modèles est calculé de la façon suivante:

$$
\text { Rang_moyen }_{k}=\frac{1}{3} *\left(\text { Rang_SAR }_{k}+\text { Rang_MSE_cotes_num }{ }_{k}+\text { Rang_corr_Spearman }_{k}\right) \text {, }
$$

où, Rang_moyen ${ }_{k}$ est le rang moyen des statistiques du modèle $k$, Rang_SAR ${ }_{k}$ est le rang du SAR du modèle $k$, Rang_MSE_cotes_num $k$ est le rang de l'erreur quadratique moyenne des cotes de crédit numériques du modèle $k$ et Rang_corr_Spearman ${ }_{k}$ est le rang de la corrélation de Spearman entre les cotes numériques estimées et les cotes numériques réelles du modèle $k$. Les rangs moyens calculés avec la formule ci-haut sont ensuite ordonnés du plus petit au plus grand. Le modèle avec le rang moyen le plus faible est considéré comme étant le meilleur. Le modèle expert à un rang de 23 pour son $S A R$, de 19 pour son erreur quadratique moyenne des cotes de crédit numériques et de 4 pour sa corrélation de Spearman entre les cotes numériques estimées et les cotes numériques réelles. Les rangs des mesures du modèle avec tout le poids sur les facteurs quantitatifs sont respectivement de 31,60 et 25 . Les résultats complets de l'analyse comparative se trouvent en annexe. 


\begin{tabular}{|c|c|c|c|c|c|c|c|}
\hline NOM DU MODĖLE & SAR & $\begin{array}{l}\text { ERREUR QUADRATIQUE } \\
\text { MOYENNE DES } \\
\text { COTES DE CRÉDIT } \\
\text { NUMÉRIQUES }\end{array}$ & $\begin{array}{l}\text { CORRÉLATION DE SPEARMAN } \\
\text { ENTRE LES COTES } \\
\text { NUMÉRIQUES ESTIMÉES ET LES } \\
\text { COTES NUMÉRIQUES RÉELLES }\end{array}$ & $\begin{array}{c}\text { RANG } \\
\text { DU SAR }\end{array}$ & $\begin{array}{l}\text { RANG DE L'ERREUR } \\
\text { QUADRATIQUE MOYENNE } \\
\text { DES COTES DE CRÉDIT } \\
\text { NUMÉRIQUES }\end{array}$ & $\begin{array}{l}\text { RANG DE LA CORRÉLATION DE } \\
\text { SPEARMAN ENTRE LES COTES } \\
\text { NUMÉRIQUES ESTIMÉES ET LES } \\
\text { COTES NUMÉRIQUES RÉELLES }\end{array}$ & RANG \\
\hline $\begin{array}{l}\text { Modèle avec } 50 \% \text { sur les facteurs } \\
\text { quantitatifs et une répartition } \\
\text { aléatoire U1 sur les facteurs } \\
\text { qualitatifs }\end{array}$ & 0,35 & 1,33 & 0,31 & 15 & 9 & 5 & 1 \\
\hline $\begin{array}{l}\text { Modèle avec } 70 \% \text { sur les facteurs } \\
\text { quantitatifs et une répartition } \\
\text { équi-pondérée sur les facteurs } \\
\text { qualitatifs }\end{array}$ & 0,35 & 1,47 & 0,32 & 8 & 31 & 2 & 2 \\
\hline $\begin{array}{l}\text { Modèle avec } 60 \% \text { sur les facteurs } \\
\text { quantitatifs et une répartition faite } \\
\text { par des experts sur les facteurs } \\
\text { qualitatifs (modèle expert) }\end{array}$ & 0,34 & 1,38 & 0,31 & 23 & 19 & 4 & 3 \\
\hline$\ldots$ & $\ldots$ & $\ldots$ & $\ldots$ & $\ldots$ & $\ldots$ & $\ldots$ & $\ldots$ \\
\hline $\begin{array}{l}\text { Modèle avec } 100 \% \text { sur les } \\
\text { facteurs quantitatifs }\end{array}$ & 0,32 & 1,71 & 0,25 & 31 & 60 & 25 & 47 \\
\hline
\end{tabular}


nettement supérieur au rang du modèle expert. Le modèle avec tout le poids sur les facteurs quantitatifs a un SAR de 0,32, une erreur quadratique moyenne des cotes de crédit numériques de 1,71 et une corrélation de Spearman entre les cotes numériques estimées et les cotes numériques réelles de 0,25. Les rangs de ces statistiques sont respectivement 31,60 et 25 . À la lumière de ces résultats, on est porté à croire que les facteurs qualitatifs ont un pouvoir explicatif sur les cotes de crédit attribuées par les agences de notations au financement de projet. De plus, les poids attribués par les experts aux facteurs prédisent mieux les cotes de crédit externes que $95 \%$ des modèles alternatifs testés.

\section{CONCLUSION}

Le but de cet essai est de répondre à la question suivante:

Quel sont les facteurs quantitatifs, macroéconomiques et qualitatifs pertinents dans un modèle de notation fantôme de financements de projet? Quels sont les poids de ces facteurs dans le modèle?

Afin de répondre à cette question, un modèle économétrique est développé. Tout d'abord, un échantillon de financements de projet est créé. Ce dernier comprend les informations financières des financements de projet. Un sous échantillon du premier comprend également les informations qualitatives sur des financements de projet. Après avoir étudié les échantillons, les différentes approches économétriques de développement de modèle de notation sont évaluées afin de déterminer laquelle est la plus appropriée pour nos échantillons. La plus adéquate s'est avérée être la méthode de notation fantôme. Cette méthode est basée sur la régression linéaire et réplique les cotes des agences de notation tel que Moody's, S\&P et DBRS.

Une fois la méthode choisie, une première étape d'analyse univariée des facteurs quantitatifs et macroéconomiques est effectuée afin de déterminer lesquels des facteurs semblent avoir un pouvoir explicatif sur la probabilité de défaut d'un financement de projet. Après cette analyse univariée, une analyse multivariée est faite pour trouver quelle est la combinaison de facteurs qui réplique le mieux les cotes des agences de notation et quel est le poids de chacun des facteurs dans cette combinaison. Suite à cette étape, un modèle comprenant seulement des facteurs quantitatifs et macroéconomiques est créé. 
La prochaine étape consiste à ajouter les facteurs qualitatifs au modèle. Par contre, comme l'échantillon est trop petit pour utiliser des méthodes économétriques traditionnelles, les poids des facteurs qualitatifs sont déterminés par des experts. Afin de tester la validité des poids des facteurs qualitatifs les performances du modèle expert sont comparées à celles de modèles alternatifs. En étudiant le SAR, l'erreur quadratique moyenne des cotes de crédit numériques et la corrélation de Spearman entre les cotes numériques estimées et les cotes numériques réelles ont conclu que le modèle expert surpasse la majorité des modèles alternatifs et surpasse grandement le modèle avec tout le poids sur les facteurs quantitatifs.

Le modèle final est présenté à la figure 30. Une prochaine étape serait d'évaluer le pouvoir explicatif des facteurs qualitatifs et leur poids respectif dans le modèle en utilisant un échantillon comprenant assez d'observations d'informations qualitatives afin de pouvoir faire des tests statistiques standard et, tout comme dans le développement de la portion quantitative du modèle, utiliser la régression linéaire pour le développement du modèle.

\section{BIBLIOGRAPHIE}

[1] Amato, Jeffery D. et Craig H. Furfine (2004). Are credit ratings procyclical?. Switzerland: Bank for International Settlements.

[2] Berblinger, J. (1996). Marktakzeptanz des Ratings durch Qualität, in Büschgen und Everling. Wiesbaden: Handbuch Rating.

[3] Bhojraj, Sanjeev Et Partha Sengupta (2003). Effect of Corporate Governance on Bond Ratings and Yields: The Role of Institutional Investors and Outside Directors. New York: Cornell University.

[4] Caranci, Michael J. et Kent Wildeman (2011). Methodology: Rating Project Finance. Toronto: DBRS.

[5] Comité de Bâle sur le contrôle bancaire (2004). Convergence internationale de la mesure et des normes de fonds propres. Bâle: Banque des Règlements Internationaux.

[6] Crarciun, Mihnea (2011). Macroeconomic and Political Risk Management in Infrastructure Projects. Ploiesti: Petroleum-Gas University of Ploiesti.

[7] Hunter, William et al. (2015). Moody's Credit Policy: Default and Recovery Rates for Project Finance Bank Loans, 1983-2013. New York: Moody's. 
[8] Delfour, Olivier, George Cherian et al (2009). Global Master Criteria Report: Rating Criteria for Infrastructure and Project Finance. New York: Fitch Ratings.

[9] Deluz, Catherine N. (2010). Moody's Investors Service Rating Methodology: Generic Project Finance Methodology. Toronto: Moody's.

[10] Ederington, Louis H. (1985). Classification models and bond ratings. Caroline du Nord: The financial review.

[11] Erlenmaier, Ulrich. "Shadow Rating Approach: Experience from Banking Practice" in Bernd Engelmann et Robert Rauhmeier. (2011). The Basel II Risk Parameters : Estimation, Validation, Stress Testing -with Applications to Loan Risk Management. New York: Springer.

[12] Evans, James D. (1996). Straightforward statistics for the behavioral sciences. Pacific Grove: Brooks/Cole Publishing Company.

[13] Falk, Frank R. et Nancy B. Miller (1992). A Primer for Soft Modeling. Akron: The University of Akron Press.

[14] Fic, Joanna (2014). Moody's Investors Service Rating Methodology: Privately Managed Toll Roads. London: Moody's.

[15] Gardner, David et James Wright. "Project Finance» in Tony Rhodes (2012). Euromoney Encyclopedia of Debt Finance Second Edition. Londre: Euromoney Books.

[16] Gevero, Arnold (2014). Trends In Global Project Finance. Manhattan: S\&P Capital IQ McGraw Hill Financial.

[17] Gini, Corrado (1912). "Variabilità e mutabilità» in Pizetti, E. et Salvemini, T. (1955). Memorie di metodologica statistica. Rome: Libreria Eredi Virgilio Veschi.

[18] Gonzales, Fernando et al. (2004). Market dynamics associated with credit ratings: a literature review, Banque de France Financial Stability Review. Germany: European Central Bank.

[19] Greene, William H. (2012). Econometric Analysis, New Jersey: Prentice Hall.

[20] Gupta, Vivek, Kusum Parwani et al. (2009). Moody's Credit Policy: Moody's Senior Ratings Algorithm \& Estimated Senior Ratings. New York: Moody's.

[21] Hull, John C. (2010). Risk Management and Financial Institutions, 2nd Edition. Toronto: Pearson. 
[22] Izzi, Luisa, Gianluca Oricchio et al (2012). Basel III Credit Rating Systems: An Applied Guide to Quantitative and Qualitative Models, Royaume Uni: Palgrave Macmillan.

[23] Jahan, Selim et al. (2015). Human Development Report 2015: Work for Human Development, New York: United Nations Development Programme.

[24] Kensinger, John W. et John D. Martin. «Project Finance: Raising Money the Old-Fashioned Way» in Donald H. Chew, Jr. (1993). The New Corporate Finance: Where Theory Meets Practice. New York: McGraw-Hill.

[25] Krahen, Jan. "Handwörterbuch des Bank- und Finanzwesens" in Gerke, W. et Steiner, M. (2001). Handwörterbuch des Bank- und Finanzwesens. Stuttgart: Schäffer-Poeschel.

[26] Lendberg, David C. et al (2014). Project Finance Operations Methodology. New York: Standard and Poor's Rating Direct.

[27] MacDonald, Ben L. et al (2013). Project Finance Default and Recovery: Shale Gas Fuels Rise In U.S. Defaults. San Francisco: Standard and Poor's Rating Direct.

[28] Matthies, Alexander B. (2013). Empirical Research on Corporate Credit-Ratings: A Literature Review. Berlin: Economic Risk.

[29] Metz, Albert et Richard Cantor (2006). Moody's Credit Rating Prediction Model. New York: Moody's.

[30] Penn State Eberly College of Science. STAT 501. URL: https:// onlinecourses.science.psu.edu/stat501/node/347 Consulté le 26 mai 2016.

[31] Rabobank (2014). QRA Rating Model Development Guidelines. Utrecht: Rabobank.

[32] Selting, Anne C. et al (2014). Project Finance Framework Methodology. San Francisco: Standard and Poor's Rating Direct.

[33] Tennant, Jennifer (2009). Moody's Credit Policy: Default and Recovery Rates for Project Finance Debts, 1992-2008. New York: Moody's.

[34] Verdouw, Win et al. (2015). Currency Risk in Project Finance. Winnipeg: International Institute for Sustainable Development.

[35] Wappenschmidt, Christian (2009). Ratinganalyse durch internatinale Ratingagenturen, Frankfurt am Main usw. Munich: Université Louiset-Maximilien de Munich.

[36] Wasserman, Larry (2005). All of Statistics: A Concise Course in Statistical Inference. New York: Springer. 
[37] Varekamp, Bart (2014). A light on the Shadow-Bond approach: The development of RI's new Commercial Banks PD model. Enschede: University of Twente.

[38] Zaalberg, Rebecca (2013). Quantifying uncertainty in credit rating model development. Enschede: University of Twente.

\section{NOTES}

1. https://www.tdsecurities.com/tds/content/CMkt_RatingsCorrelation?language=fr_CA\& changeLanguage $=Y$

2. Dans Wasserman (2005), une p-value supérieure à 0.1 veut dire qu'il n'y a pas de présomption contre l'hypothèse nulle. Dans cet essai le seuil de $10 \%$ sera donc utilisé afin de préserver tous les facteurs qui pourraient avoir une présomption contre l'hypothèse nulle.

3. Comme le facteur marge EBIT est significatif à $10 \%$ sans variable indicatrice d secteur PPP et infra et que ce facteur aide à comprendre les coûts réels d'affaires de la gestion de la compagnie, il est conservé pour l'analyse multivariée. 